\title{
Rotational spectroscopy, tentative interstellar detection, and chemical modeling of $\mathrm{N}$-methylformamide ${ }^{\star}$
}

\author{
A. Belloche ${ }^{1}$, A. A. Meshcheryakov², R. T. Garrod ${ }^{3}$, V. V. Ilyushin² ${ }^{2}$ E. A. Alekseev²,4, \\ R. A. Motiyenko ${ }^{5}$, L. Margulès ${ }^{5}$, H. S. P. Müller ${ }^{6}$, and K. M. Menten ${ }^{1}$

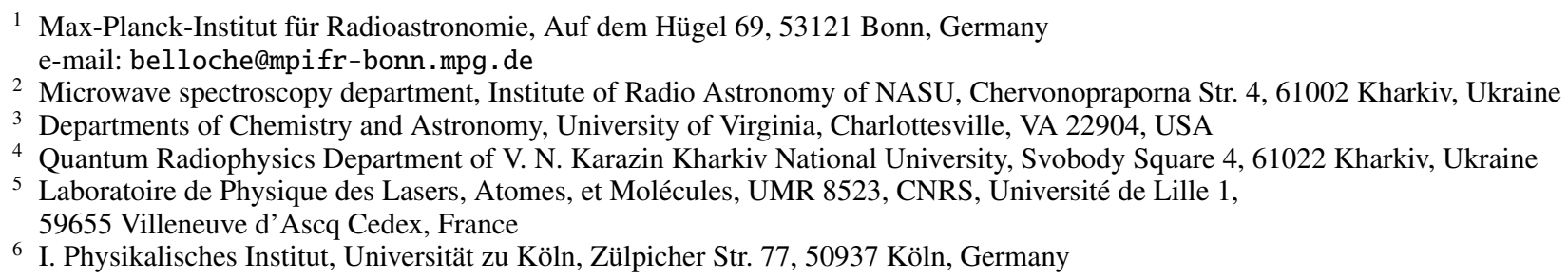

Received 16 September 2016 / Accepted 17 January 2017

\begin{abstract}
Context. N-methylformamide, $\mathrm{CH}_{3} \mathrm{NHCHO}$, may be an important molecule for interstellar pre-biotic chemistry because it contains a peptide bond, which in terrestrial chemistry is responsible for linking amino acids in proteins. The rotational spectrum of the most stable trans conformer of $\mathrm{N}$-methylformamide is complicated by strong torsion-rotation interaction due to the low barrier of the methyl torsion. For this reason, the theoretical description of the rotational spectrum of the trans conformer has, up to now, not been accurate enough to provide a firm basis for its interstellar detection.

Aims. In this context, as a prerequisite for a successful interstellar detection, our goal is to improve the characterization of the rotational spectrum of N-methylformamide.

Methods. We use two absorption spectrometers in Kharkiv and Lille to measure the rotational spectra over the frequency range 45$630 \mathrm{GHz}$. The analysis is carried out using the Rho-axis method and the RAM36 code. We search for N-methylformamide toward the hot molecular core Sagittarius (Sgr) B2(N2) using a spectral line survey carried out with the Atacama Large Millimeter/submillimeter Array (ALMA). The astronomical spectra are analyzed under the assumption of local thermodynamic equilibrium. The astronomical results are put into a broader astrochemical context with the help of a gas-grain chemical kinetics model.

Results. The new laboratory data set for the trans conformer of N-methylformamide consists of 9469 distinct line frequencies with $J \leq 62$, including the first assignment of the rotational spectra of the first and second excited torsional states. All these lines are fitted within experimental accuracy for the first time. Based on the reliable frequency predictions obtained in this study, we report the tentative detection of $\mathrm{N}$-methylformamide toward Sgr B2(N2). We find N-methylformamide to be more than one order of magnitude less abundant than formamide $\left(\mathrm{NH}_{2} \mathrm{CHO}\right)$, a factor of two less abundant than the unsaturated molecule methyl isocyanate $\left(\mathrm{CH} \mathrm{H}_{3} \mathrm{NCO}\right)$, but only slightly less abundant than acetamide $\left(\mathrm{CH}_{3} \mathrm{CONH}_{2}\right)$. We also report the tentative detection of the ${ }^{15} \mathrm{~N}$ isotopolog of formamide $\left({ }^{15} \mathrm{NH}_{2} \mathrm{CHO}\right)$ toward Sgr B2(N2). The chemical models indicate that the efficient formation of $\mathrm{HNCO}$ via $\mathrm{NH}+\mathrm{CO}$ on grains is a necessary step in the achievement of the observed gas-phase abundance of $\mathrm{CH}_{3} \mathrm{NCO}$. Production of $\mathrm{CH}_{3} \mathrm{NHCHO}$ may plausibly occur on grains either through the direct addition of functional-group radicals or through the hydrogenation of $\mathrm{CH}_{3} \mathrm{NCO}$.

Conclusions. Provided the detection of N-methylformamide is confirmed, the only slight underabundance of this molecule compared to its more stable structural isomer acetamide and the sensitivity of the model abundances to the chemical kinetics parameters suggest that the formation of these two molecules is controlled by kinetics rather than thermal equilibrium.
\end{abstract}

Key words. line: identification - molecular data - radio lines: ISM - ISM: molecules - ISM: individual objects: Sagittarius B2(N) astrochemistry

\section{Introduction}

The peptide bond is a fundamental building block of life on Earth (Kaiser et al. 2013). Therefore, peptide molecules have for a long time attracted much attention. The simplest molecule containing a peptide bond, formamide $\left(\mathrm{NH}_{2} \mathrm{CHO}\right)$, was detected in the interstellar medium (ISM) back in the 1970s (Rubin et al. 1971). The relatively high abundance of formamide also permitted the detection of rotational lines of its first excited vibrational

* Full Tables 2 and 3 are only available at the CDS via anonymous ftp to cdsarc.u-strasbg. fr (130.79.128.5) or via http://cdsarc.u-strasbg.fr/viz-bin/qcat?]/A+A/601/A49 state $v_{12}=1$ in Orion KL (Motiyenko et al. 2012) and in Sagittarius (Sgr) B2(N) (Belloche et al. 2013). N-methylformamide, $\mathrm{CH}_{3} \mathrm{NHCHO}$, is one of the simplest derivatives of formamide and also a peptide molecule. It is of interest as a candidate for interstellar detection because its structural isomer acetamide $\left(\mathrm{CH}_{3} \mathrm{CONH}_{2}\right)$ has already been detected in the ISM (Hollis et al. 2006; Halfen et al. 2011). $\mathrm{CH}_{3} \mathrm{NHCHO}$ is the second most stable $\mathrm{C}_{2} \mathrm{H}_{5} \mathrm{NO}$ isomer after acetamide (Lattelais et al. 2010).

$\mathrm{N}$-methylformamide exists in two stable rotameric forms, trans and cis. Their structures are shown in Fig. 1. According to quantum chemical calculations, the trans conformer is more stable than cis by $466 \mathrm{~cm}^{-1}$ (666 K, Kawashima et al. 2010). 


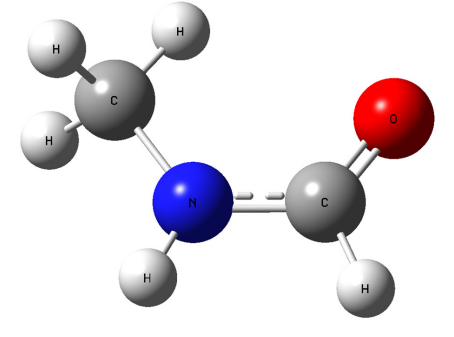

(a)

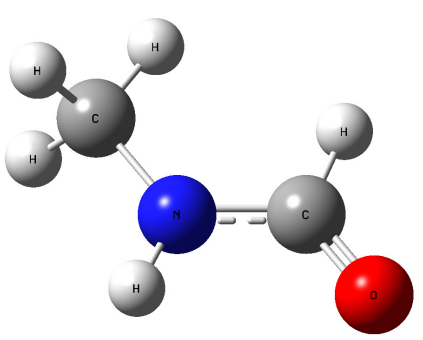

(b)
Fig. 1. Structure of trans (a) and cis (b) conformations of N-methylformamide.

The trans conformer is also characterized by a very low barrier to internal rotation of the methyl top. The coupling between the overall rotation of the molecule and the almost free rotation of the methyl top significantly complicates the description of the spectrum. For this reason, N-methylformamide has been the subject of extensive spectroscopic investigations, but for a long time the analysis of its microwave rotational spectrum did not yield satisfactory results. Fantoni \& Caminati (1996) were the first to succeed in assigning rotational spectral lines belonging to the trans conformer in the $\mathrm{CH}_{3}$ internal rotation ground state of $A$ symmetry. They performed measurements between 18 and $40 \mathrm{GHz}$, but could not identify any spectral lines of the $E$ species. Later, Fantoni et al. (2002) published results of measurements and analysis for $E$ lines. The $V_{3}$ barrier of the methyl group internal rotation was determined to be $55.17 \pm 0.84 \mathrm{~cm}^{-1}$.

Recently, Kawashima et al. (2010) carried out a new spectroscopic investigation of N-methylformamide. In that study, rotational spectra of both trans and cis conformers of the normal as well as deuterated $\mathrm{CH}_{3} \mathrm{NHCHO}$ isotopologs were measured in the frequency range of 5-118 GHz. Molecular parameters including rotational constants and $V_{3}$ barriers to methyl-group internal rotation were determined for all investigated species and conformers. Owing to the relatively high barrier to internal rotation for the cis conformer, a good description was obtained for its 108 measured transitions. However, the low barrier and the limitations in the model used for the theoretical description of the rotational spectrum allowed these authors to fit only low $J$ quantum number transitions $(J<11)$ for the trans conformer. In addition, 60 out of 467 assigned transitions of the trans conformer were excluded from the final fit as their residuals ranged from 1 to $67 \mathrm{MHz}$ and were much higher than the experimental accuracy estimated to be $0.004-0.05 \mathrm{MHz}$.

One should note that in previous publications, two different schemes were used for naming the conformations of N-methylformamide. Fantoni \& Caminati (1996) and Fantoni et al. (2002) used the dihedral angle $D\left(\mathrm{H}-\mathrm{N}-\mathrm{C}-\mathrm{H}^{\prime}\right)$ where $\mathrm{H}^{\prime}$ is the carbonyl group hydrogen. The conformer with $D=0^{\circ}$ was named $\mathrm{cis}$ and the conformer with $D=180^{\circ}$ was named trans. Kawashima et al. (2010) used another convention widely accepted for molecules with a peptide bond. According to this convention, applied for $\mathrm{N}$-methylformamide, one should use the $D^{*}(\mathrm{Y}-\mathrm{N}-\mathrm{C}-\mathrm{X})$ dihedral angle, where $\mathrm{X}$ is the carbonyl hydrogen and $\mathrm{Y}$ is the methyl group. Cis and trans conformers named using this convention are thus the opposite of the cis and trans conformers in Fantoni \& Caminati (1996) and Fantoni et al. (2002). Here we use the naming adopted by Kawashima et al. (2010), that is, using the $D^{*}$ dihedral angle.
In the present study, we extend the measurement and analysis of the rotational spectrum of trans $\mathrm{N}$-methylformamide, which is of higher interest for astrophysical detection. We use the accurate frequency predictions obtained in this study to search for N-methylformamide in the ISM. We target the high mass star-forming region Sgr B2(N), one of the most prolific sources for the detection of complex organic molecules in the ISM (e.g., Belloche et al. 2013). For this, we use a spectral survey of Sgr B2(N) conducted with the Atacama Large Millimeter/submillimeter Array (ALMA) in its Cycles 0 and 1. This survey aims at exploring molecular complexity with ALMA (EMoCA, see Belloche et al. 2016).

The experimental setup is presented in Sect. 2. The analysis of the rotational spectrum of N-methylformamide and the results that follow are described in Sect. 3. A tentative detection of N-methylformamide in Sgr B2(N) is presented in Sect. 4 along with the derivation of column densities of other related molecules of interest. Chemical modeling is performed in Sect. 5 to put the observational results into a broader astrochemical context. The results are discussed in Sect. 6 and the conclusions are presented in Sect. 7.

\section{Experimental setup}

A sample of N-methylformamide was purchased from SigmaAldrich and used without further purification. The experimental investigation of the absorption spectrum of $\mathrm{N}$-methylformamide was carried out over the frequency range 45-630 $\mathrm{GHz}$ using two microwave spectrometers. The first one is the automated millimeter wave spectrometer of the Institute of Radio Astronomy of NASU in Kharkiv, Ukraine (Alekseev et al. 2012). The second one is the terahertz spectrometer of the Laboratory of Physics of Lasers, Atoms, and Molecules in Lille, France (Zakharenko et al. 2015).

The spectrometer in Kharkiv is built according to the classical scheme of absorption spectrometers, and its detailed description can be found in Alekseev et al. (2012). The spectrometer was slightly upgraded with the aim of expanding the operating frequency range; a new backward-wave oscillator (BWO) unit covering frequencies from 34 to $52 \mathrm{GHz}$ has been put into operation. Thus, at present, this spectrometer can record spectra between 34 and $250 \mathrm{GHz}$. In order to improve the sensitivity below $50 \mathrm{GHz}$, a new waveguide absorbing cell (a copper waveguide of $10 \times 72 \mathrm{~mm}^{2}$ internal cross section and $295 \mathrm{~cm}$ length) was used instead of the commonly employed quasi-optic absorption cell. The measurements of $\mathrm{N}$-methylformamide with the Kharkiv spectrometer were done in the frequency range 45 to $150 \mathrm{GHz}$. All measurements were performed at room temperature and with sample pressures (approximately 10 mTorr) that provided close to Doppler-limited spectral resolution. The frequency determination errors were estimated to be 10,30 , and $100 \mathrm{kHz}$ depending on the measured signal-to-noise ratio.

The measurements in Lille were performed between 150 and $630 \mathrm{GHz}$ at typical pressures of $10 \mathrm{~Pa}$ and at room temperature. The frequency determination errors were estimated to be $30 \mathrm{kHz}$ and $50 \mathrm{kHz}$ below and above $330 \mathrm{GHz}$, respectively. The frequencies of the lines with poor signal-to-noise ratio or distorted lineshape were measured with $50 \mathrm{kHz}$ or $100 \mathrm{kHz}$ accuracy.

\section{Spectroscopic analysis and results}

We performed the analysis using the Rho-axis method (RAM), which was already applied successfully to a number of 
molecules with large-amplitude torsional motion. The method uses the axis system obtained by rotation of the principal axis system to make the new $z$ axis parallel to the $\rho$ vector. The coordinates of the $\rho$ vector are calculated using the following expression:

$\rho_{g}=\frac{\lambda_{g} I_{\alpha}}{I_{g}},(g=x, y, z)$,

where $\lambda_{g}$ are the direction cosines of the internal rotation axis of the top in the principal axis system, $I_{g}$ are the principal inertia moments, and $I_{\alpha}$ is the inertia moment of the methyl top. The RAM Hamiltonian may be written as (Kleiner 2010):

$H_{\mathrm{RAM}}=H_{\mathrm{T}}+H_{\mathrm{R}}+H_{\mathrm{cd}}+H_{\mathrm{int}}$.

$H_{\mathrm{T}}$ represents the torsional Hamiltonian defined as:

$H_{\mathrm{T}}=F\left(p_{\alpha}-\rho J_{z}\right)^{2}+V(\alpha)$,

where $F$ is the internal rotation constant, $p_{\alpha}$ is the internal rotation angular momentum conjugate to the torsion angle $\alpha$, and $V(\alpha)$ is the internal rotation potential function:

$V(\alpha)=\frac{1}{2} V_{3}(1-\cos 3 \alpha)+\frac{1}{2} V_{6}(1-\cos 6 \alpha)+\ldots$

$H_{\mathrm{R}}$ represents the rigid rotor rotational Hamiltonian in the rhoaxis system. In addition to usual $A, B$, and $C$ terms, for the molecules with $(x z)$ plane of symmetry (as is appropriate for $\mathrm{N}$-methylformamide), it contains a non-diagonal term $D_{x z}$. In the $I^{r}$ coordinate representation, $H_{\mathrm{R}}$ has the following form:

$H_{\mathrm{R}}=A_{\mathrm{RAM}} J_{z}^{2}+B_{\mathrm{RAM}} J_{x}^{2}+C_{\mathrm{RAM}} J_{y}^{2}+D_{x z}\left(J_{x} J_{z}+J_{z} J_{x}\right)$.

The last two terms in Eq. (1), $H_{\mathrm{cd}}$ and $H_{\text {int }}$, are the usual centrifugal distortion and higher-order torsional-rotational interaction Hamiltonians, respectively.

To fit and predict the rotational spectra, we used the RAM36 (Rho-axis method for 3 and 6-fold barriers) program that allows one to include in the model almost any symmetryallowed torsion-rotation Hamiltonian term up to the twelfth order (Ilyushin et al. 2010, 2013). The RAM Hamiltonian in Eq. (1) may be expressed in the following form used in the RAM36 program :

$$
\begin{aligned}
H= & \frac{1}{2} \sum_{k n p q r s} B_{k n p q r s 0}\left[J^{2 k} J_{z}^{n} J_{x}^{p} J_{y}^{q} p_{\alpha}^{r} \cos (3 s \alpha)\right. \\
& \left.+\cos (3 s \alpha) p_{\alpha}^{r} J_{y}^{q} J_{x}^{p} J_{z}^{n} J^{2 k}\right] \\
& +\frac{1}{2} \sum_{k n p q r t} B_{k n p q r 0 t}\left[J^{2 k} J_{z}^{n} J_{x}^{p} J_{y}^{q} p_{\alpha}^{r} \sin (3 t \alpha)\right. \\
& \left.+\sin (3 t \alpha) p_{\alpha}^{r} J_{y}^{q} J_{x}^{p} J_{z}^{n} J^{2 k}\right]
\end{aligned}
$$

where the $B_{\text {knpqrst }}$ are fitting parameters. In the case of a $C_{3 v}$ top and $C_{s}$ frame (as is appropriate for $\mathrm{N}$-methylformamide), the allowed terms in the torsion-rotation Hamiltonian must be totally symmetric in the group $G_{6}$ (and also must be Hermitian and invariant to the time reversal operation). Since all individual operators $p_{\alpha}, J_{x}, J_{y}, J_{z}, J^{2}, \cos (3 s \alpha)$, and $\sin (3 t \alpha)$ used in Eq. (5) are Hermitian, all possible terms provided by Eq. (5) will automatically be Hermitian. The particular term to be fitted is represented in the input file with a set of $k, n, p, q, r, s, t$ integer indices that are checked by the program for conformity with time reversal and symmetry requirements, to prevent accidental introduction of symmetry-forbidden terms into the Hamiltonian. For example, $B_{0000200}$ corresponds to $F$ in Eq. (2), $B_{0200000}$ to $A_{\mathrm{RAM}}$ in Eq. (4), etc. In Table 1, which presents the final set of molecular parameters, we give, instead of $B_{\text {knpqrst }}$, more conventional names for the parameters whose nomenclature is based on the subscript procedures of $\mathrm{Xu}$ et al. (2008).

The RAM36 program uses the free-rotor quantum number $m$ to label the torsional energy levels. It is well known that the internal rotation of a methyl top attached to a molecular frame should be treated as an anharmonic vibrational motion well below the top of the barrier to internal rotation, and as a nearly free internal rotation motion well above the top of the barrier. The $v_{\mathrm{t}}$ labeling assumes that the spacings between degenerate and non-degenerate levels of the torsional Hamiltonian associated with a given $v_{\mathrm{t}}$ are much smaller than those between levels with different $v_{\mathrm{t}}$ (Lin \& Swalen 1959). The trans conformation of N-methylformamide represents an intermediate case because of the relatively low barrier to internal rotation (Lin \& Swalen 1959). Owing to the low barrier, the $A-E$ splitting in the first excited torsional state $v_{\mathrm{t}}=1$ of the trans conformation is comparable with the energy difference between the $v_{\mathrm{t}}=0$ and $v_{\mathrm{t}}=1$ states, whereas the $v_{\mathrm{t}}=2$ state lies well above the barrier. Because of this intermediate situation we decided to keep the quantum number labeling of torsional states $m$ in Tables 2 and 3, which is used inside the RAM36 program.

The RAM36 code was modified to take into account the quadrupole hyperfine structure of the transitions that is present in the spectrum of N-methylformamide due to the non-zero electric quadrupole moment of the nitrogen atom. We used the standard hyperfine energy expression:

$$
\begin{aligned}
E_{h f} & =\left[\chi_{a a}\left\langle J_{a}^{2}\right\rangle+\chi_{b b}\left\langle J_{b}^{2}\right\rangle-\left(\chi_{a a}+\chi_{b b}\right)\left\langle J_{c}^{2}\right\rangle\right. \\
& \left.+\chi_{a b}\left\langle J_{a} J_{b}+J_{b} J_{a}\right\rangle\right] \frac{2 f(I, J, F)}{J(J+1)},
\end{aligned}
$$

where $f(I, J, F)$ is the Casimir function. Typically, a resolved pattern of the hyperfine structure was observed as a doublet with an approximately two-to-one ratio in intensities. The stronger doublet component contains unresolved hyperfine transitions with selection rules $F=J+1 \leftarrow F=J+1$ and $F=J-1 \leftarrow$ $F=J-1$, whereas the weaker doublet component corresponds to the $F=J \leftarrow F=J$ transition.

We started our analysis of the trans $\mathrm{N}$-methylformamide spectrum from refitting the data available from the literature (Fantoni \& Caminati 1996; Fantoni et al. 2002; Kawashima et al. 2010) using the RAM36 code. Application of the RAM36 code allowed us to fit, almost within experimental error, the data available in the literature including those lines that were previously excluded from the fits due to relatively high observed-minus-calculated values (Kawashima et al. 2010). Thus we obtained a reliable basis for assigning the newly measured lines in the $45-630 \mathrm{GHz}$ range. Assigning and fitting of the new data using the RAM36 program proceeded in a fairly conventional, iterative way going up in frequency.

The complete data set treated at the final stage of the current study includes both our new data and data from the literature (Fantoni \& Caminati 1996; Fantoni et al. 2002; Kawashima et al. 2010). The data set contains $12456 A$ - and $E$-type transitions with $J \leq 62$ and $K_{a} \leq 21$ for trans $\mathrm{N}$-methylformamide in the lowest three torsional states. Due to blending, these 12456 transitions correspond to 9469 distinct line frequencies (mainly due to non-fully resolved quadrupole hyperfine structure). The fit chosen as the "best" uses a model consisting of 103 parameters. The weighted root-mean-square 
Table 1. Molecular parameters of the trans conformer of N-methylformamide obtained with the RAM36 program.

\begin{tabular}{|c|c|c|c|}
\hline$n t r^{a}$ & Parameter $^{b}$ & Operator $^{c}$ & Value $^{d}$ \\
\hline 220 & $F$ & $p_{\alpha}^{2}$ & $5.5825023(37)$ \\
\hline 220 & $V_{3}$ & $\frac{1}{2}(1-\cos 3 \alpha)$ & $51.7199088(90)$ \\
\hline 211 & $\rho$ & $J_{z} p_{\alpha}$ & $0.080976579(84)$ \\
\hline 202 & $A_{\mathrm{RAM}}-0.5\left(B_{\mathrm{RAM}}+C_{\mathrm{RAM}}\right)$ & $J_{z}^{2}$ & $0.3540036(48)$ \\
\hline 202 & $0.5\left(B_{\mathrm{RAM}}+C_{\mathrm{RAM}}\right)$ & $J^{2}$ & $0.22175219(40)$ \\
\hline 202 & $0.5\left(B_{\text {RAM }}-C_{\text {RAM }}\right)$ & $J_{x}^{2}-J_{u}^{2}$ & $0.05717595(45)$ \\
\hline 202 & $D_{z x}$ & $\left\{J_{z}, J_{x}^{y}\right\}$ & $-0.155070742(38)$ \\
\hline 440 & $F_{m}$ & $p_{\alpha}^{4}$ & $-0.67979(73) \times 10^{-3}$ \\
\hline 440 & $V_{6}$ & $\frac{1}{2}(1-\cos 6 \alpha)$ & $8.02866(40)$ \\
\hline 431 & $\rho_{m}$ & $J_{z} p_{\alpha}^{3}$ & $0.222402(74) \times 10^{-3}$ \\
\hline 422 & $F_{J}$ & $J^{2} p_{\alpha}^{2}$ & $-0.1029(15) \times 10^{-6}$ \\
\hline 422 & $F_{K}$ & $J_{z}^{2} p_{\alpha}^{2}$ & $-0.63309(14) \times 10^{-4}$ \\
\hline 422 & $F_{x y}$ & $p_{\alpha}^{2}\left(J_{x}^{2}-J_{y}^{2}\right)$ & $-0.23266(15) \times 10^{-5}$ \\
\hline 422 & $F_{z x}$ & $\frac{1}{2} p_{\alpha}^{2}\left\{J_{z}, J_{x}\right\}$ & $0.226160(59) \times 10^{-4}$ \\
\hline 422 & $V_{3 J}$ & $J^{2}(1-\cos 3 \alpha)$ & $-0.15939626(49) \times 10^{-2}$ \\
\hline 422 & $V_{3 K}$ & $J_{z}^{2}(1-\cos 3 \alpha)$ & $0.8718486(73) \times 10^{-2}$ \\
\hline 422 & $V_{3 z x}$ & $\frac{1}{2}(1-\cos 3 \alpha)\left\{J_{z}, J_{x}\right\}$ & $-0.9181495(26) \times 10^{-2}$ \\
\hline 422 & $V_{3 x y}$ & $\left(J_{x}^{2}-J_{y}^{2}\right)(1-\cos 3 \alpha)$ & $-0.194794(44) \times 10^{-4}$ \\
\hline 422 & $D_{3 x y}$ & $\frac{1}{2} \sin 3 \alpha\left\{J_{x}, J_{y}\right\}$ & $0.235469(41) \times 10^{-3}$ \\
\hline 413 & $\rho_{J}$ & $J^{2} J_{z} p_{\alpha}$ & $0.240348(50) \times 10^{-5}$ \\
\hline 413 & $\rho_{K}$ & $J_{z}^{3} p_{\alpha}$ & $0.112304(16) \times 10^{-4}$ \\
\hline 413 & $\rho_{z x}$ & $\frac{1}{2} p_{\alpha}\left\{J_{z}^{2}, J_{x}\right\}$ & $-0.186678(14) \times 10^{-4}$ \\
\hline 413 & $\rho_{x y}$ & $\frac{1}{2} p_{\alpha}\left\{J_{z},\left(J_{x}^{2}-J_{u}^{2}\right)\right\}$ & $0.473931(43) \times 10^{-5}$ \\
\hline 404 & $D_{z x K}$ & $\left\{J_{z}^{3}, J_{x}\right\}$ & $0.203044(10) \times 10^{-5}$ \\
\hline 404 & $\Delta_{J}$ & $-J^{4}$ & $0.300775(22) \times 10^{-6}$ \\
\hline 404 & $\Delta_{J K}$ & $-J^{2} J_{z}^{2}$ & $-0.83337(25) \times 10^{-6}$ \\
\hline 404 & $\Delta_{K}$ & $-J_{z}^{4^{z}}$ & $0.302147(39) \times 10^{-5}$ \\
\hline 404 & $\delta_{J}$ & $-2 J^{2}\left(J_{x}^{2}-J_{y}^{2}\right)$ & $0.108271(13) \times 10^{-6}$ \\
\hline 404 & $\delta_{K}$ & $-\left\{J_{z}^{2},\left(J_{x}^{2}-J_{y}^{2}\right)\right\}$ & $0.367453(69) \times 10^{-6}$ \\
\hline 660 & $F_{m m}$ & $p_{\alpha}^{6}$ & $-0.10119(30) \times 10^{-4}$ \\
\hline 660 & $V_{9}$ & $\frac{1}{2}(1-\cos 9 \alpha)$ & $2.0575(23)$ \\
\hline 651 & $\rho_{m m}$ & $p_{\alpha}^{5} J_{z}$ & $0.26713(46) \times 10^{-5}$ \\
\hline 642 & $F_{m J}$ & $J^{2} p_{\alpha}^{4}$ & $0.3393(19) \times 10^{-7}$ \\
\hline 642 & $F_{m K}$ & $J_{z}^{2} p_{\alpha}^{4}$ & $-0.6445(16) \times 10^{-6}$ \\
\hline 642 & $F_{m x y}$ & $p_{\alpha}^{4}\left(J_{x}^{2}-J_{y}^{2}\right)$ & $0.2157(17) \times 10^{-7}$ \\
\hline 642 & $F_{m z x}$ & $\frac{1}{2} p_{\alpha}^{4}\left\{J_{z}, J_{x}\right\}$ & $-0.1406(36) \times 10^{-7}$ \\
\hline 642 & $V_{6 J}$ & $J^{2}(1-\cos 6 \alpha)$ & $-0.124130(82) \times 10^{-3}$ \\
\hline 642 & $V_{6 K}$ & $J_{z}^{2}(1-\cos 6 \alpha)$ & $0.4820(10) \times 10^{-3}$ \\
\hline 642 & $V_{6 z x}$ & $\frac{1}{2}(1-\cos 6 \alpha)\left\{J_{z}, J_{x}\right\}$ & $-0.543394(97) \times 10^{-3}$ \\
\hline 642 & $V_{6 x y}$ & $(1-\cos 6 \alpha)\left(J_{x}^{2}-J_{y}^{2}\right)$ & $-0.20435(93) \times 10^{-4}$ \\
\hline 642 & $D_{6 x y}$ & $\frac{1}{2} \sin 6 \alpha\left\{J_{x}, J_{y}\right\}$ & $0.6217(56) \times 10^{-4}$ \\
\hline 642 & $D_{6 z y}$ & $\frac{1}{2} \sin 6 \alpha\left\{J_{z}, J_{y}\right\}$ & $-0.12563(23) \times 10^{-3}$ \\
\hline 633 & $\rho_{m J}$ & $J^{2} p_{\alpha}^{3} J_{z}$ & $-0.3725(33) \times 10^{-8}$ \\
\hline 633 & $\rho_{m K}$ & $J_{z}^{3} p_{\alpha}^{3}$ & $0.5362(28) \times 10^{-7}$ \\
\hline 633 & $\rho_{m x y}$ & $\frac{1}{2} p_{\alpha}^{3}\left\{J_{z},\left(J_{x}^{2}-J_{y}^{2}\right)\right\}$ & $-0.819(25) \times 10^{-9}$ \\
\hline 633 & $\rho_{m z x}$ & $\frac{1}{2} p_{\alpha}^{3}\left\{J_{z}^{2}, J_{x}\right\}$ & $-0.1058(18) \times 10^{-7}$ \\
\hline 624 & $F_{J J}$ & $J^{4} p_{\alpha}^{2}$ & $0.2173(36) \times 10^{-10}$ \\
\hline 624 & $F_{K K}$ & $J_{z}^{4} p_{\alpha}^{2}$ & $-0.2049(35) \times 10^{-8}$ \\
\hline 624 & $F_{z x K}$ & $\frac{1}{2} p_{\alpha}^{2}\left\{J_{z}^{3}, J_{x}\right\}$ & $-0.1137(15) \times 10^{-8}$ \\
\hline 624 & $V_{3 J J}$ & $J^{4}(1-\cos 3 \alpha)$ & $0.131102(86) \times 10^{-7}$ \\
\hline 624 & $V_{3 J K}$ & $J^{2} J_{z}^{2}(1-\cos 3 \alpha)$ & $0.15148(95) \times 10^{-7}$ \\
\hline
\end{tabular}

Notes. ${ }^{(a)} n=t+r$, where $n$ is the total order of the operator, $t$ is the order of the torsional part and $r$ is the order of the rotational part, respectively. (b) Parameter nomenclature based on the subscript procedures of Xu et al. (2008) ${ }^{(c)}\{A, B, C\}=A B C+C B A,\{A, B\}=A B+B A$. The product of the operator in the third column of a given row and the parameter in the second column of that row gives the term actually used in the torsion-rotation Hamiltonian of the program, except for $F, \rho$ and $A_{\mathrm{RAM}}$, which occur in the Hamiltonian in the form $F\left(p_{\alpha}-\rho P_{a}\right)^{2}+A_{\mathrm{RAM}} P_{a}^{2}$. ${ }^{(a)}$ All values are in $\mathrm{cm}^{-1}$ (except $\rho$ which is unitless). Statistical uncertainties are shown as one standard uncertainty in the units of the last two digits. 
A. Belloche et al.: Rotational spectroscopy, tentative interstellar detection, and chemical modeling of N-methylformamide

Table 1. continued.

\begin{tabular}{|c|c|c|c|}
\hline$n t r^{a}$ & Parameter $^{b}$ & Operator ${ }^{c}$ & Value $^{d}$ \\
\hline 624 & $V_{3 K K}$ & $J_{z}^{4}(1-\cos 3 \alpha)$ & $-0.5940(31) \times 10^{-7}$ \\
\hline 624 & $V_{3 z x J}$ & $\frac{1}{2} J^{2}(1-\cos 3 \alpha)\left\{J_{z}, J_{x}\right\}$ & $0.3290(72) \times 10^{-8}$ \\
\hline 624 & $V_{3 x y J}$ & $J^{2}(1-\cos 3 \alpha)\left(J_{x}^{2}-J_{y}^{2}\right)$ & $-0.2988(10) \times 10^{-8}$ \\
\hline 624 & $V_{3 x y K}$ & $\frac{1}{2}(1-\cos 3 \alpha)\left\{J_{z}^{2},\left(J_{x}^{2}-J_{y}^{2}\right)\right\}$ & $-0.1819(10) \times 10^{-7}$ \\
\hline 624 & $V_{3 z x K}$ & $\frac{1}{2}(1-\cos 3 \alpha)\left\{J_{z}^{3}, J_{x}\right\}$ & $-0.9915(12) \times 10^{-7}$ \\
\hline 624 & $V_{3 z x x}$ & $\frac{1}{2} \cos 3 \alpha\left\{J_{z}, J_{x}^{3}\right\}$ & $-0.15343(15) \times 10^{-6}$ \\
\hline 624 & $V_{3 x 4 y 4}$ & $\cos 3 \alpha\left(J_{x}^{4}+J_{y}^{4}\right)$ & $0.7525(14) \times 10^{-8}$ \\
\hline 624 & $D_{3 x y J}$ & $\frac{1}{2} J^{2} \sin 3 \alpha\left\{J_{x}, J_{y}\right\}$ & $-0.6130(23) \times 10^{-8}$ \\
\hline 624 & $D_{3 x y K}$ & $\frac{1}{2} \sin 3 \alpha\left\{J_{z}^{2}, J_{x}, J_{y}\right\}$ & $0.23854(49) \times 10^{-6}$ \\
\hline 624 & $D_{3 z y J}$ & $\frac{1}{2} J^{2} \sin 3 \alpha\left\{J_{z}, J_{y}\right\}$ & $-0.6514(19) \times 10^{-7}$ \\
\hline 624 & $D_{3 z y K}$ & $\frac{1}{2} \sin 3 \alpha\left\{J_{z}^{3}, J_{y}\right\}$ & $-0.5861(61) \times 10^{-7}$ \\
\hline 624 & $D_{3 z y y}$ & $\frac{1}{2} \sin 3 \alpha\left\{J_{z}, J_{y}^{3}\right\}$ & $0.8302(24) \times 10^{-7}$ \\
\hline 624 & $D_{3 x 3 y x y 3}$ & $\frac{1}{2} \sin 3 \alpha\left[\left\{J_{x}^{3}, J_{y}\right\}-\left\{J_{x}, J_{y}^{3}\right\}\right]$ & $0.4008(24) \times 10^{-8}$ \\
\hline 615 & $\rho_{J J}$ & $J^{4} J_{z} p_{\alpha}$ & $-0.1203(18) \times 10^{-10}$ \\
\hline 615 & $\rho_{J K}$ & $J^{2} J_{z}^{3} p_{\alpha}$ & $-0.6148(28) \times 10^{-9}$ \\
\hline 615 & $\rho_{K K}$ & $J_{z}^{5} p_{\alpha}$ & $0.4502(53) \times 10^{-9}$ \\
\hline 615 & $\rho_{z x J}$ & $\frac{1}{2} J^{2} p_{\alpha}\left\{J_{z}^{2}, J_{x}\right\}$ & $0.475(11) \times 10^{-10}$ \\
\hline 615 & $\rho_{z x K}$ & $\frac{1}{2} p_{\alpha}\left\{J_{z}^{4}, J_{x}\right\}$ & $0.13926(45) \times 10^{-8}$ \\
\hline 615 & $\rho_{x 2 y 2}$ & $\frac{1}{2} p_{\alpha}\left\{J_{z}, J_{x}^{2}, J_{y}^{2}\right\}$ & $0.23934(84) \times 10^{-9}$ \\
\hline 606 & $D_{z x K K}$ & $\left\{J_{z}^{5}, J_{x}\right\}$ & $-0.7781(20) \times 10^{-10}$ \\
\hline 606 & $\Phi_{J}$ & $J^{6}$ & $0.4542(10) \times 10^{-12}$ \\
\hline 606 & $\Phi_{K J}$ & $J^{2} J_{z}^{4}$ & $0.3394(14) \times 10^{-10}$ \\
\hline 606 & $\Phi_{K}$ & $J_{z}^{6^{2}}$ & $0.2619(35) \times 10^{-10}$ \\
\hline 606 & $\phi_{J}$ & $2 J^{4}\left(J_{x}^{2}-J_{y}^{2}\right)$ & $0.23367(53) \times 10^{-12}$ \\
\hline 606 & $\phi_{J K}$ & $J^{2}\left\{J_{z}^{2},\left(J_{x}^{2}-J_{y}^{2}\right)\right\}$ & $-0.6207(42) \times 10^{-12}$ \\
\hline 606 & $\phi_{K}$ & $\left\{J_{z}^{4},\left(J_{x}^{2}-J_{y}^{2}\right)\right\}$ & $0.22553(51) \times 10^{-10}$ \\
\hline 871 & $\rho_{m m m}$ & $J_{z} p_{\alpha}^{7}$ & $0.7121(15) \times 10^{-7}$ \\
\hline 862 & $F_{m m J}$ & $J^{2} p_{\alpha}^{6}$ & $0.4570(73) \times 10^{-9}$ \\
\hline 862 & $F_{m m K}$ & $J_{z}^{2} p_{\alpha}^{6}$ & $-0.19488(81) \times 10^{-7}$ \\
\hline 862 & $F_{m m x y}$ & $p_{\alpha}^{6}\left(J_{x}^{2}-J_{y}^{2}\right)$ & $0.3816(70) \times 10^{-9}$ \\
\hline 862 & $V_{9 J}$ & $J^{2}(1-\cos 9 \alpha)$ & $-0.15061(48) \times 10^{-3}$ \\
\hline 862 & $V_{9 K}$ & $J_{z}^{2}(1-\cos 9 \alpha)$ & $0.1550(58) \times 10^{-3}$ \\
\hline 862 & $V_{9 x y}$ & $(1-\cos 9 \alpha)\left(J_{x}^{2}-J_{y}^{2}\right)$ & $-0.5022(55) \times 10^{-4}$ \\
\hline 862 & $D_{9 x y}$ & $\frac{1}{2} \sin 9 \alpha\left\{J_{x}, J_{y}\right\}$ & $-0.1195(32) \times 10^{-3}$ \\
\hline 853 & $\rho_{m m K}$ & $J_{z}^{3} p_{\alpha}^{5}$ & $0.2984(20) \times 10^{-8}$ \\
\hline 853 & $\rho_{m m z x}$ & $\frac{1}{2} p_{\alpha}^{5}\left\{J_{z}^{2}, J_{x}\right\}$ & $-0.411(11) \times 10^{-9}$ \\
\hline 844 & $F_{m J K}$ & $J^{2} J_{z}^{2} p_{\alpha}^{4}$ & $0.980(55) \times 10^{-11}$ \\
\hline 844 & $F_{m K K}$ & $J_{z}^{4} p_{\alpha}^{4}$ & $-0.2444(30) \times 10^{-9}$ \\
\hline 844 & $F_{m x y K}$ & $\frac{1}{2} p_{\alpha}^{4}\left\{J_{z}^{2},\left(J_{x}^{2}-J_{y}^{2}\right)\right\}$ & $0.1692(29) \times 10^{-10}$ \\
\hline 844 & $V_{6 z x x}$ & $\frac{1}{2} \cos 6 \alpha\left\{J_{z}, J_{x}^{3}\right\}$ & $-0.1034(16) \times 10^{-7}$ \\
\hline 844 & $D_{6 x y J}$ & $\frac{1}{2} J^{2} \sin 6 \alpha\left\{J_{x}, J_{y}\right\}$ & $-0.1224(35) \times 10^{-8}$ \\
\hline 844 & $D_{6 z y K}$ & $\frac{1}{2} \sin 6 \alpha\left\{J_{z}^{3}, J_{y}\right\}$ & $0.573(14) \times 10^{-7}$ \\
\hline 835 & $\rho_{m K K}$ & $J_{z}^{5} p_{\alpha}^{3}$ & $0.1312(35) \times 10^{-10}$ \\
\hline 826 & $F_{K K K}$ & $J_{z}^{6} p_{\alpha}^{2}$ & $-0.491(25) \times 10^{-12}$ \\
\hline 826 & $V_{3 J J K}$ & $J^{4} J_{z}^{2}(1-\cos 3 \alpha)$ & $-0.365(13) \times 10^{-12}$ \\
\hline 826 & $V_{3 K K K}$ & $J_{z}^{6}(1-\cos 3 \alpha)$ & $-0.909(34) \times 10^{-11}$ \\
\hline 826 & $V_{3 z x x x}$ & $\frac{1}{2} \cos 3 \alpha\left\{J_{z}, J_{x}^{5}\right\}$ & $0.675(14) \times 10^{-12}$ \\
\hline 826 & $D_{3 x y J K}$ & $\frac{1}{2} J^{2} \sin 3 \alpha\left\{J_{z}^{2}, J_{x}, J_{y}\right\}$ & $-0.1837(65) \times 10^{-11}$ \\
\hline \multirow[t]{4}{*}{826} & $D_{3 x y K K}$ & $\frac{1}{2} \sin 3 \alpha\left\{J_{z}^{4}, J_{x}, J_{y}\right\}$ & $-0.1309(33) \times 10^{-10}$ \\
\hline & $\chi_{a a}$ & $0.70093(90) \times 10^{-4}$ & \\
\hline & $\chi_{b b}$ & $0.64466(92) \times 10^{-4}$ & \\
\hline & $2 \chi_{a b}$ & $0.1755(15) \times 10^{-4}$ & \\
\hline
\end{tabular}


deviation of the fit of 12456 microwave transition frequencies with $J \leq 62$ is 0.84 , indicating that assumed statistical uncertainties were slightly overestimated. The largest residual of $0.293 \mathrm{MHz}$ is observed in the fit for the $v_{\mathrm{t}}=0 \mathrm{E}$-symmetry species transition $49_{9,41} \leftarrow 48_{9,40}$. The final set of molecular parameters is presented in Table 1 . The final data set of fitted transitions of the $\mathrm{N}$-methylformamide trans conformer is presented in Table 2, where we provide quantum numbers for each level, followed by observed transition frequencies, measurement uncertainties, and residuals from the fit. The complete version of Table 2 is available at the CDS; here only part of the table is presented for illustration purposes.

Comparison of the low-order parameter values from Table 1 with the corresponding parameters determined by Kawashima et al. (2010) reveals relatively significant shifts in the values on a background of a general qualitative agreement (see Table B.1). Part of these shifts come from the basic difference in the models used, although both refer to the Rho-axis method. We follow the definition of the Rho-axis method given in Hougen et al. (1994) and, in our case, the $A$ and $E$ species are treated together with a single set of rotational parameters. On the contrary, Kawashima et al. (2010) treated the $A$ and $E$ species with distinct sets of rotational parameters (see their Table 2). As a result, a direct comparison of the changes in the rotational parameters is not possible. The same is true for the centrifugal distortion constants. At the same time, the change in $V_{3}$ value is in good agreement with the prediction made in Kawashima et al. (2010) where a relatively large $V_{6}$ term in the potential function was postulated by analogy with acetamide (Ilyushin et al. 2004). The data set available in Kawashima et al. (2010) was limited to the ground torsional state transitions only and did not give the opportunity to determine the $V_{6}$ value. Thus, they examined how the $V_{3}$ potential barrier is changed when a $V_{6}$ term is added, assuming that the coupling of the vibrational modes of the $\mathrm{NH}$ group with the $\mathrm{CH}_{3}$ internal rotation has an effect similar to the one observed for the $\mathrm{NH}_{2}$ group in acetamide (Hirota et al. 2010). According to our results, the value of an index $R$ used in Kawashima et al. (2010) to express the effect of the $V_{6}$ term quantitatively is estimated to be 26.5 which is in a relatively good agreement with the value of 23.0 obtained in Kawashima et al. (2010) on the basis of ground state data only. Thus, our results support the general analysis of the $\mathrm{CH}_{3}$ internal rotation potential barrier in $\mathrm{N}$-methylformamide provided in Kawashima et al. (2010).

A portion of the rotational spectrum of $\mathrm{N}$-methylformamide measured around $133 \mathrm{GHz}$ in the laboratory is shown in Fig. 2 and compared to the predicted rotational spectrum as provided by our current theoretical model. As can be seen from Fig. 2, the overall correspondence between experimental and theoretical spectra is very good. A slight inconsistency with intensity between predicted and observed spectra that may be visible for some strong lines is due to variations of source power and detector sensitivity.

The predictions of rotational transitions of trans $\mathrm{N}$-methylformamide in the $v_{\mathrm{t}}=0,1$, and 2 torsionally excited states resulting from the fit are presented in Table 3. They are calculated for the frequency range up to $650 \mathrm{GHz}$ and for the transitions with $J \leq 65$. The table provides quantum numbers, followed by calculated transition frequencies and their uncertainties, the energy of the lower state and the product $\mu^{2} S$, where $\mu$ is the dipole moment of the molecule and $S$ is the line strength of the transition. Owing to its significant size, the complete version of Table 3 is available at the CDS.

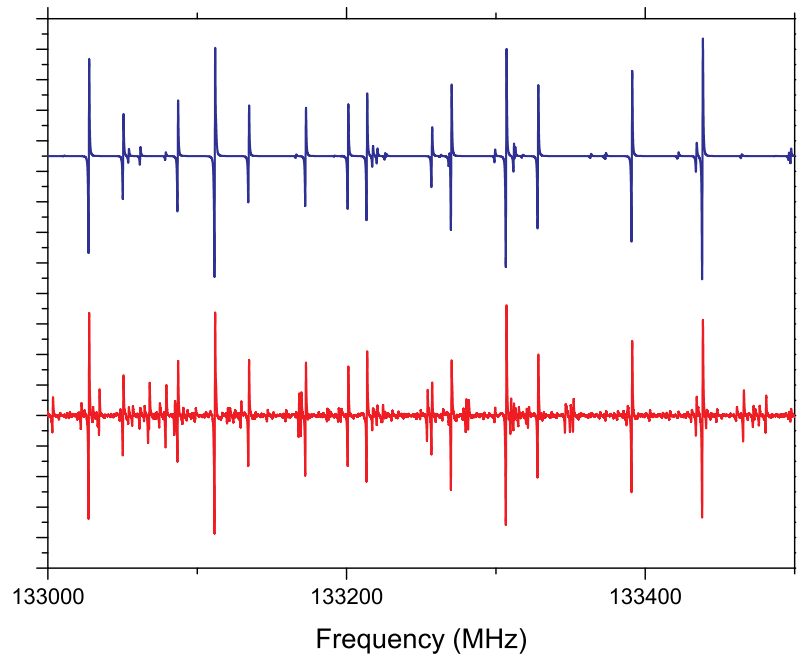

Fig. 2. Predicted (upper panel) and measured (lower panel) rotational spectrum of N-methylformamide between 133 and $133.5 \mathrm{GHz}$. The observed line shapes correspond to the first derivative of the actual line profile because frequency modulation and first harmonic lock-in detection are employed. The intensity axis is in arbitrary units.

We provide the torsional-rotational $\left(Q_{\mathrm{tr}}\right)$ and vibrational $\left(Q_{\mathrm{v}}\right)$ partition functions of $\mathrm{N}$-methylformamide in Table 4 . The values of $Q_{\mathrm{tr}}$ were calculated from first principles, that is, via direct summation over the rotational-torsional states. The maximum value of the $J$ quantum number of the energy levels taken into account to calculate the partition function is 130 , and excited torsional states up to $v_{\mathrm{t}}=8$ were considered. The vibrational part, $Q_{\mathrm{v}}$, was estimated using a harmonic approximation and a simple formula that may be found in Gordy \& Cook (1984, see their Eq. 3.60). The frequencies of the normal modes were obtained from DFT calculations of the harmonic force field using the B3LYP method and a $6-311++(3 \mathrm{df}, 2 \mathrm{pd})$ basis set. Table 4 also lists the partition function values of acetamide that we calculated in a similar way as for $\mathrm{N}$-methylformamide. The torsionalrotational part was calculated from first principales on the basis of the results presented in the paper by Ilyushin et al. (2004). To compute the $Q_{\mathrm{v}}$ values, we used, when available, vibrational frequencies reported in the literature (Kutzelnigg \& Mecke 1962; Kydd \& Dunham 1980), but also the results of DFT calculations with the same method and basis set as for N-methylformamide. For both molecules, the values of $Q_{\mathrm{v}}$ were calculated by taking all the vibrational modes into account except for the torsional mode which is already considered in $Q_{\mathrm{tr}}$. The full partition function, $Q_{\text {tot }}$, is thus the product of $Q_{\mathrm{tr}}$ and $Q_{v}$.

\section{Astronomical results}

\subsection{Observations}

We use the full data set of the EMoCA spectral line survey obtained toward Sgr B2(N) with ALMA in its Cycles 0 and 1. The survey covers the frequency range between 84.1 and $114.4 \mathrm{GHz}$ with a spectral resolution of $488.3 \mathrm{kHz}\left(1.7\right.$ to $\left.1.3 \mathrm{~km} \mathrm{~s}^{-1}\right)$. The median angular resolution is $1.6^{\prime \prime}$. A detailed description of the observations, the data reduction process, and the method used to identify the detected lines and derive column densities was presented in Belloche et al. (2016). Population diagrams are constructed in the same way as in our previous work. Here, we would like to emphasize the fact that the apparent discrepancy between the synthetic populations and the fit to the observed 
Table 2. Measured transitions of trans $\mathrm{N}$-methylformamide in the $v_{t}=0,1$, and 2 states.

\begin{tabular}{ccccccccccccc}
\hline \hline$m^{\prime}$ & $F^{\prime a}$ & $J^{\prime}$ & $K_{a}^{\prime}$ & $K_{c}^{\prime}$ & $m^{\prime \prime}$ & $F^{\prime \prime a}$ & $J^{\prime \prime}$ & $K_{a}^{\prime \prime}$ & $K_{c}^{\prime \prime}$ & $\begin{array}{c}\text { Obs. freq. } \\
(\mathrm{MHz})\end{array}$ & $\begin{array}{c}\text { Uncertainty } \\
(\mathrm{MHz})\end{array}$ & $\begin{array}{c}\text { Obs.-calc. } \\
(\mathrm{MHz})\end{array}$ \\
\hline-3 & 18 & 19 & 3 & 16 & -3 & 17 & 18 & 2 & 16 & 251285.2870 & 0.1000 & 0.0203 \\
-3 & 20 & 19 & 3 & 16 & -3 & 19 & 18 & 2 & 16 & 251285.2870 & 0.1000 & -0.0235 \\
-3 & 19 & 19 & 3 & 16 & -3 & 18 & 18 & 2 & 16 & 251286.1600 & 0.1000 & 0.0037 \\
3 & & 21 & 4 & 17 & 3 & & 20 & 5 & 16 & 251379.8920 & 0.0500 & 0.0007 \\
-2 & & 23 & 3 & 21 & -2 & & 22 & 3 & 20 & 251386.7420 & 0.0500 & -0.0109 \\
-2 & & 23 & 3 & 21 & -2 & & 22 & 3 & 20 & 251386.7430 & 0.0500 & -0.0099 \\
3 & & 22 & 2 & 21 & 3 & & 21 & 2 & 19 & 251468.4440 & 0.0500 & 0.0002 \\
0 & & 22 & 8 & 15 & 0 & & 21 & 8 & 14 & 251472.2980 & 0.0500 & -0.0016 \\
0 & & 22 & 8 & 14 & 0 & & 21 & 8 & 13 & 251493.1300 & 0.0500 & 0.0136 \\
1 & & 22 & 8 & 15 & 1 & & 21 & 8 & 14 & 251565.7320 & 0.0500 & 0.0034 \\
0 & 18 & 19 & 4 & 16 & 0 & 17 & 18 & 3 & 15 & 251591.3020 & 0.0500 & 0.0035 \\
0 & 20 & 19 & 4 & 16 & 0 & 19 & 18 & 3 & 15 & 251591.3020 & 0.0500 & -0.0310 \\
0 & 19 & 19 & 4 & 16 & 0 & 18 & 18 & 3 & 15 & 251591.9750 & 0.0500 & -0.0035 \\
\hline
\end{tabular}

Notes. The complete table is available at the CDS. ${ }^{(a)}$ The quantum number $F$ is not indicated for the transitions with unresolved hyperfine structure.

Table 3. Predicted transitions of trans $\mathrm{N}$-methylformamide in the $v_{t}=0,1$, and 2 states.

\begin{tabular}{cccccccccccccc}
\hline \hline$m^{\prime}$ & $F^{\prime}$ & $J^{\prime}$ & $K_{\mathrm{a}}^{\prime}$ & $K_{\mathrm{c}}^{\prime}$ & $m^{\prime \prime}$ & $F^{\prime \prime}$ & $J^{\prime \prime}$ & $K_{\mathrm{a}}^{\prime \prime}$ & $K_{\mathrm{c}}^{\prime \prime}$ & $\begin{array}{c}\text { Calc. freq. } \\
(\mathrm{MHz})\end{array}$ & $\begin{array}{c}\text { Uncertainty } \\
(\mathrm{MHz})\end{array}$ & $\begin{array}{c}E_{1} \\
\left(\mathrm{~cm}^{-1}\right)\end{array}$ & $\begin{array}{c}\mu^{2} S \\
\left(\mathrm{D}^{2}\right)\end{array}$ \\
\hline 1 & 30 & 30 & 15 & 15 & 1 & 30 & 30 & 14 & 17 & 393571.2045 & 0.0384 & 266.1079 & 20.6 \\
1 & 31 & 30 & 15 & 15 & 1 & 31 & 30 & 14 & 17 & 393571.2531 & 0.0384 & 266.1079 & 21.3 \\
1 & 29 & 30 & 15 & 15 & 1 & 29 & 30 & 14 & 17 & 393571.2547 & 0.0384 & 266.1079 & 20.0 \\
0 & 24 & 24 & 5 & 19 & 0 & 24 & 24 & 3 & 22 & 393583.8360 & 0.0055 & 115.5411 & 0.0382 \\
0 & 25 & 24 & 5 & 19 & 0 & 25 & 24 & 3 & 22 & 393585.7457 & 0.0055 & 115.5411 & 0.0398 \\
0 & 23 & 24 & 5 & 19 & 0 & 23 & 24 & 3 & 22 & 393585.8253 & 0.0055 & 115.5411 & 0.0367 \\
0 & 15 & 15 & 8 & 8 & 0 & 15 & 15 & 6 & 9 & 393600.5805 & 0.0054 & 62.7047 & 0.0364 \\
0 & 16 & 15 & 8 & 8 & 0 & 16 & 15 & 6 & 9 & 393600.7174 & 0.0054 & 62.7047 & 0.0389 \\
0 & 14 & 15 & 8 & 8 & 0 & 14 & 15 & 6 & 9 & 393600.7265 & 0.0054 & 62.7047 & 0.0342 \\
-3 & 41 & 41 & 3 & 38 & -3 & 40 & 40 & 3 & 37 & 393611.4811 & 0.0389 & 337.7355 & 36.9 \\
-3 & 42 & 41 & 3 & 38 & -3 & 41 & 40 & 3 & 37 & 393611.5587 & 0.0389 & 337.7355 & 37.8 \\
-3 & 40 & 41 & 3 & 38 & -3 & 39 & 40 & 3 & 37 & 393611.5617 & 0.0389 & 337.7355 & 36.0 \\
\hline
\end{tabular}

Notes. The complete table is available at the CDS.

populations, the former lying below the latter (see, e.g., Fig 4b), results from the fact that the model is optimized to match (i.e., not overestimate) the peak temperatures of the detected transitions while the population diagram is based on integrated intensities. Because of the high level of line confusion, the wings of the detected lines are often still partially contaminated even after removing the contribution of the other known molecules included in our complete model. Therefore, the populations derived from the integrated intensities are, most of the time, slightly overestimated.

The complete model mentioned above refers to the synthetic spectrum that includes the emission of all the molecules that we have identified in Sgr B2(N2) so far (Belloche et al. 2013, 2014, 2016; Müller et al. 2016a,b; Margulès et al. 2016); it is overlaid in green in all figures that display observed spectra.

We focus our analysis on the secondary hot core Sgr B2(N2), which is located $\sim 5^{\prime \prime}$ to the North of the main hot core Sgr B2(N1) (Belloche et al. 2016). Sgr B2(N2) has narrower linewidths $\left(F W H M \sim 5 \mathrm{~km} \mathrm{~s}^{-1}\right)$ and thus exhibits a lower degree of line confusion than Sgr B2(N1). While some of the molecules reported below are also present on larger scales in the envelope of Sgr B2 (see, e.g., Jones et al. 2008), our interferometric observations are only sensitive to the compact emission arising from the embedded hot cores. As shown in Fig. 3, the emission analyzed in this section is compact and the derived column densities refer to the hot core Sgr B2(N2) only.

\subsection{Tentative detection of $\mathrm{N}$-methylformamide $\left(\mathrm{CH}_{3} \mathrm{NHCHO}\right)$}

We searched for emission lines of $\mathrm{CH}_{3} \mathrm{NHCHO}$ toward Sgr B2(N2) using the spectroscopic predictions obtained in Sect. 3. We compared the observed ALMA spectrum of Sgr B2(N2) to synthetic spectra of $\mathrm{CH}_{3} \mathrm{NHCHO}$ produced under the assumption of local thermodynamic equilibrium (LTE), which is expected to be valid given the high densities of the hot core regions probed by the EMoCA survey (Belloche et al. 2016). Figures A.1-A.3 show all the transitions of $\mathrm{CH}_{3} \mathrm{NHCHO}$ in its ground state and its first and second torsionally excited states that are covered by our survey and are expected to contribute significantly to the detected signal for typical hot core temperatures (150-200 K). In these figures, the synthetic spectrum containing the contribution of all molecules that we have identified toward Sgr B2(N2) so far, including $\mathrm{CH}_{3} \mathrm{NHCHO}$, is overlaid in green on the observed spectrum, while the red spectrum shows the contribution of $\mathrm{CH}_{3} \mathrm{NHCHO}$ only, as derived from our best-fit LTE model. Most transitions of $\mathrm{CH}_{3} \mathrm{NHCHO}$ 
Table 4. Partition functions of N-methylformamide and acetamide.

\begin{tabular}{|c|c|c|c|c|}
\hline \multirow[b]{2}{*}{$\begin{array}{c}T \\
(\mathrm{~K})\end{array}$} & \multicolumn{2}{|c|}{$\mathrm{CH}_{3} \mathrm{NHCHO}$} & \multicolumn{2}{|c|}{$\mathrm{CH}_{3} \mathrm{CONH}_{2}$} \\
\hline & $Q_{\operatorname{tr}^{a}}$ & $Q_{\mathrm{v}}{ }^{b}$ & $Q_{\operatorname{tr}}^{a}$ & $Q_{\mathrm{V}}{ }^{b}$ \\
\hline 10 & 365.16247 & 1.00000 & 359.18058 & 1.00000 \\
\hline 20 & 1189.23944 & 1.00000 & 1272.66459 & 1.00000 \\
\hline 30 & 2488.15240 & 1.00000 & 2766.98655 & 1.00000 \\
\hline 40 & 4317.90735 & 1.00008 & 4871.11731 & 1.00009 \\
\hline 50 & 6701.57116 & 1.00058 & 7590.48345 & 1.00058 \\
\hline 60 & 9648.68076 & 1.00224 & 10925.10798 & 1.00205 \\
\hline 70 & 13164.31826 & 1.00592 & 14874.85986 & 1.00508 \\
\hline 80 & 17251.81144 & 1.01233 & 19439.98556 & 1.01017 \\
\hline 90 & 21913.57312 & 1.02193 & 24620.89439 & 1.01769 \\
\hline 100 & 27151.45535 & 1.03496 & 30418.00805 & 1.02792 \\
\hline 110 & 32966.94532 & 1.05150 & 36831.70759 & 1.04108 \\
\hline 120 & 39361.28161 & 1.07156 & 43862.32239 & 1.05734 \\
\hline 130 & 46335.51849 & 1.09509 & 51510.12815 & 1.07686 \\
\hline 140 & 53890.55428 & 1.12204 & 59775.34167 & 1.09979 \\
\hline 150 & 62027.13560 & 1.15237 & 68658.10939 & 1.12626 \\
\hline 160 & 70745.84645 & 1.18606 & 78158.49050 & 1.15642 \\
\hline 170 & 80047.08923 & 1.22311 & 88276.43644 & 1.19042 \\
\hline 180 & 89931.06287 & 1.26356 & 99011.76908 & 1.22844 \\
\hline 190 & 100397.74181 & 1.30747 & 110364.15950 & 1.27064 \\
\hline 200 & 111446.85817 & 1.35493 & 122333.10876 & 1.31725 \\
\hline 210 & 123077.88845 & 1.40605 & 134917.93177 & 1.36849 \\
\hline 220 & 135290.04535 & 1.46097 & 148117.74490 & 1.42463 \\
\hline 230 & 148082.27451 & 1.51987 & 161931.45737 & 1.48594 \\
\hline 240 & 161453.25592 & 1.58293 & 176357.76649 & 1.55275 \\
\hline 250 & 175401.40932 & 1.65036 & 191395.15645 & 1.62543 \\
\hline 260 & 189924.90280 & 1.72242 & 207041.90022 & 1.70434 \\
\hline 270 & 205021.66401 & 1.79936 & 223296.06409 & 1.78994 \\
\hline 280 & 220689.39314 & 1.88148 & 240155.51446 & 1.88269 \\
\hline 290 & 236925.57717 & 1.96908 & 257617.92629 & 1.98311 \\
\hline 300 & 253727.50471 & 2.06251 & 275680.79287 & 2.09175 \\
\hline 310 & 271092.28113 & 2.16213 & 294341.43638 & 2.20925 \\
\hline 320 & 289016.84348 & 2.26834 & 313597.01910 & 2.33626 \\
\hline 330 & 307497.97493 & 2.38156 & 333444.55472 & 2.47352 \\
\hline
\end{tabular}

Notes. ${ }^{(a)} Q_{\mathrm{tr}}$ is the torsional-rotational partition function. It does not take the hyperfine splitting into account. ${ }^{(b)} Q_{\mathrm{v}}$ is the vibrational partition function. The total partition function of the molecule (without hyperfine splitting) is $Q_{\mathrm{tr}} \times Q_{\mathrm{v}}$.

are blended with lines emitted by other molecules and therefore cannot be unambiguously assigned to $\mathrm{CH}_{3} \mathrm{NHCHO}$. However, several lines are relatively free of contamination and match lines detected toward Sgr B2(N2), both in terms of linewidths and peak temperatures. These lines are marked with a star in Col. 11 of Tables B.2-B.4. In total, five lines can be clearly assigned to $\mathrm{CH}_{3} \mathrm{NHCHO}$, four within its torsional ground state and one within its first torsionally excited state. Given the small number of clearly detected lines, we consider our detection of $\mathrm{CH}_{3} \mathrm{NHCHO}$ as tentative rather than secure.

Gaussian fits to the integrated intensity maps of four of the five detected lines indicate that the size of the emission is smaller than the beam. We obtain a size of $\sim 1.8^{\prime \prime}$ for the line at $93.41 \mathrm{GHz}$ observed in setup S3 but this is also the setup with the worst angular resolution $\left(2.9^{\prime \prime} \times 1.5^{\prime \prime}\right)$; we therefore do not fully trust this size. Indeed the other lines, at 91.89, 99.69 (see Fig. 3a), and $113.61 \mathrm{GHz}$, are all unresolved, suggesting a size smaller than $1^{\prime \prime}$. We adopt a size of $0.9^{\prime \prime}$ for our LTE modeling of $\mathrm{CH}_{3} \mathrm{NHCHO}$. We did not attempt to fit a size to the integrated

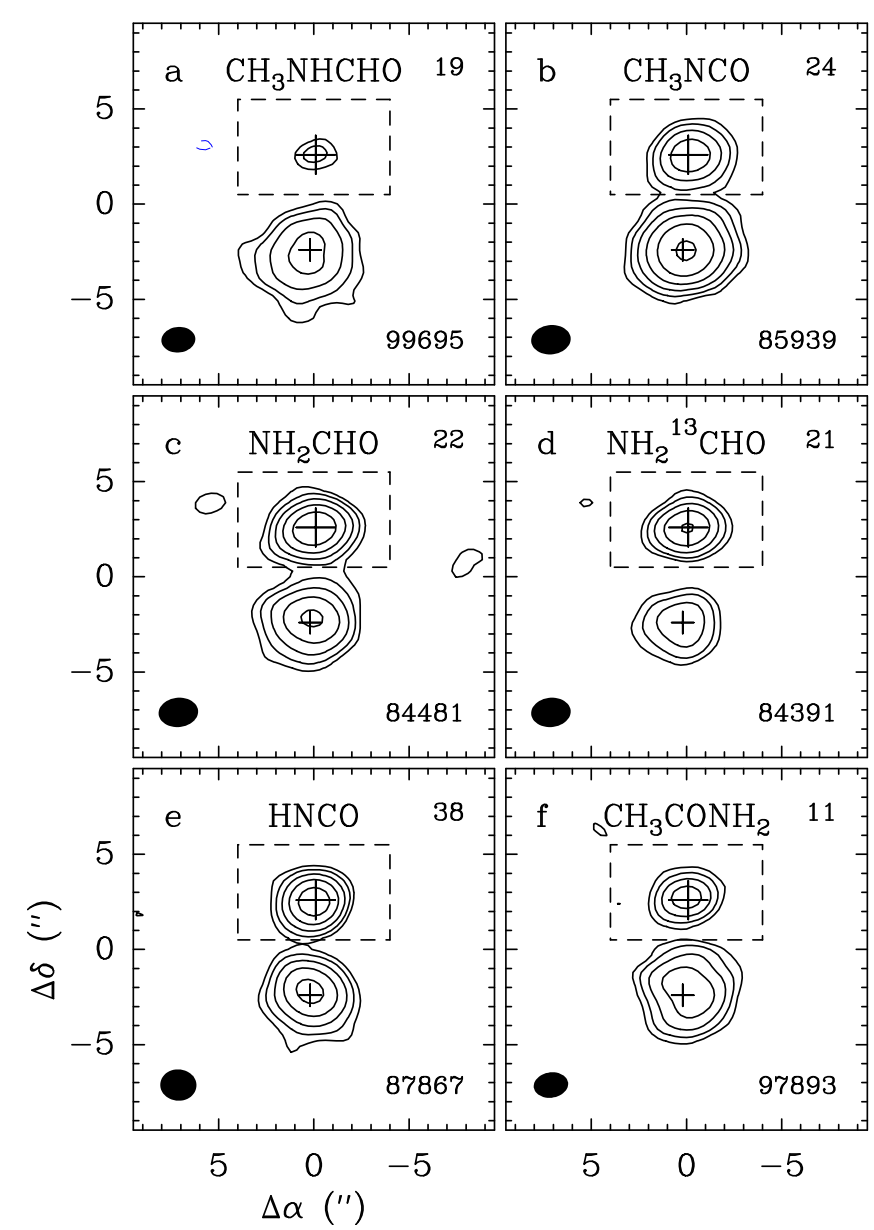

Fig. 3. Integrated intensity maps toward $\mathrm{Sgr} \mathrm{B} 2(\mathrm{~N} 2)$ of a) $\mathrm{CH}_{3} \mathrm{NHCHO}$ $9_{2,8}-8_{2,7}$, b) $\mathrm{CH}_{3} \mathrm{NCO} \quad 10_{1,10}-9_{1,9}$, c) $\mathrm{NH}_{2} \mathrm{CHO} v_{12}=14_{0,4}$ $3_{0,3}$, d) $\mathrm{NH}_{2}{ }^{13} \mathrm{CHO} 4_{0,4}-3_{0,3}$, e) $\mathrm{HNCO} 4_{3,1}-3_{3,0}$ and $4_{3,2}-3_{3,1}$, and f) $\mathrm{CH}_{3} \mathrm{CONH}_{2} 9_{0,9}-8_{1,8}, 9_{1,9}-8_{1,8}, 9_{0,9}-8_{0,8}$, and $9_{1,9}-8_{0,8}$. The position of Sgr B2(N2) is marked with a large cross. The smaller cross indicates the position of Sgr B2(N1). Because of the different systemic velocities of the two sources, the line assignment is only valid for Sgr B2(N2), inside the dashed box. The contours start at $4 \sigma$ and increase by a factor of 2 at each step. The dashed (blue) contour is at $-4 \sigma$. The value of the rms noise level, $\sigma$, is given in mJy beam ${ }^{-1} \mathrm{~km} \mathrm{~s}^{-1}$ in the top right corner of each panel. The mean frequency of the integration range in $\mathrm{MHz}$ and the synthesized beam are shown in the bottom right and left corners of each panel, respectively.

intensity map of the fifth detected line, at $111.22 \mathrm{GHz}$, because it is somewhat more contaminated on its low-frequency side.

The population diagram of $\mathrm{CH}_{3} \mathrm{NHCHO}$ is shown in Fig. 4. We used all detected lines plus a number of other ones that contribute significantly to detected lines and are contaminated by species that we have already identified and included in our full model. All the transitions used for the population diagram are listed in Tables B.2-B.4. Panel b of Fig. 4 shows the population diagram after correcting for the optical depth of the transitions, based on our best-fit LTE model of $\mathrm{CH}_{3} \mathrm{NHCHO}$, and after removing the contamination by other species, based on our full model. A fit to this diagram yields a rotational temperature of $149 \pm 20 \mathrm{~K}$, with a significant uncertainty (Table 5). For the LTE modeling of the spectrum, we adopt a temperature of $180 \mathrm{~K}$. The best-fit parameters are listed in Table 6 .

In the following sections, we derive the column density of molecules that may be related to $\mathrm{N}$-methylformamide in order to 
A. Belloche et al.: Rotational spectroscopy, tentative interstellar detection, and chemical modeling of N-methylformamide
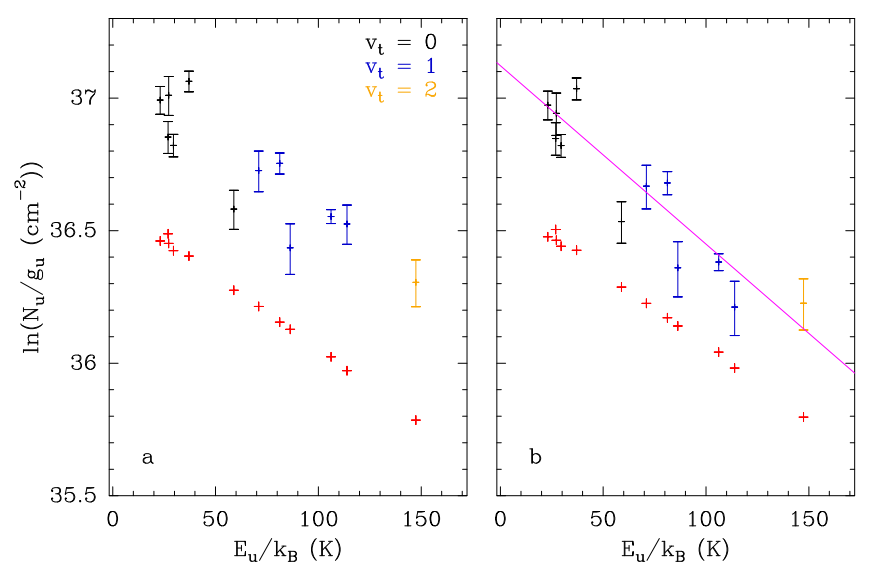

Fig. 4. Population diagram of $\mathrm{CH}_{3} \mathrm{NHCHO}$ toward $\mathrm{Sgr} \mathrm{B} 2(\mathrm{~N} 2)$. The observed datapoints are shown in various colors (but not red) as indicated in the upper right corner of panel a) while the synthetic populations are shown in red. No correction is applied in panel a). In panel b), the optical depth correction has been applied to both the observed and synthetic populations and the contamination by all other species included in the full model has been removed from the observed datapoints. The purple line is a linear fit to the observed populations (in linear-logarithmic space).

Table 5. Rotational temperatures derived from population diagrams of selected complex organic molecules toward Sgr B2(N2).

\begin{tabular}{lll}
\hline \hline \multicolumn{1}{c}{ Molecule } & \multicolumn{1}{c}{ States $^{a}$} & \multicolumn{1}{c}{$\begin{array}{c}T_{\mathrm{fit}}{ }^{b} \\
(\mathrm{~K})\end{array}$} \\
\hline $\mathrm{CH}_{3} \mathrm{NHCHO}$ & $v_{\mathrm{t}}=0, v_{\mathrm{t}}=1, v_{\mathrm{t}}=2$ & $149(20)$ \\
\hline $\mathrm{CH}_{3} \mathrm{NCO}$ & $v_{\mathrm{b}}=0, v_{\mathrm{b}}=1$ & $140.9(7.2)$ \\
\hline $\mathrm{NH}_{2} \mathrm{CHO}$ & $v=0, v_{12}=1$ & $154.8(3.3)$ \\
$\mathrm{NH}_{2}{ }^{13} \mathrm{CHO}$ & $v=0, v_{12}=1$ & $222(37)$ \\
\hline $\mathrm{HNCO}_{\mathrm{CH}_{3} \mathrm{CONH}}$ & $v=0, v_{5}=1, v_{6}=1, v_{4}=1$ & $220.1(9.6)$ \\
\hline
\end{tabular}

Notes. ${ }^{(a)}$ Vibrational or torsional states that were taken into account to fit the population diagram. ${ }^{(b)}$ The standard deviation of the fit is given in parentheses. As explained in Sect. 3 of Belloche et al. (2016), these uncertainties are purely statistical and should be viewed with caution. They may be underestimated.

put the tentative detection of this molecule into a broader astrochemical context.

\subsection{Methyl isocyanate $\left(\mathrm{CH}_{3} \mathrm{NCO}\right)$}

The first interstellar detection of methyl isocyanate was obtained toward Sgr B2(N) based on single-dish observations (Halfen et al. 2015, ; see also Cernicharo et al. 2016), shortly after its in-situ detection in the frozen surface of comet 67P/Churyumov-Gerasimenko (Goesmann et al. 2015). Here, we use the predictions available in the Cologne database for molecular spectroscopy ${ }^{1}$ (CDMS, Müller et al. 2005) (tags 57505 and 57 506, both version 1), which are based on measurements reported by Cernicharo et al. (2016) and Koput (1986).

Methyl isocyanate is well detected toward Sgr B2(N2) in the EMoCA spectral survey: 60 lines are clearly detected in its vibrational ground state and four in its first vibrationally excited state (Figs. A.4 and A.5). Gaussian fits to the integrated intensity maps of the detected lines indicate a median emission size

\footnotetext{
1 http://www.astro.uni-koeln.de/cdms/
}

of $\sim 1.2^{\prime \prime}$ with a rms dispersion of $\sim 0.1^{\prime \prime}$ (see Fig. $3 \mathrm{~b}$ ). A fit to its population diagram shown in Fig. A.20 yields a rotational temperature of $141 \pm 7 \mathrm{~K}$ (Table 5). We adopt a temperature of $150 \mathrm{~K}$ for our LTE modeling. The parameters of our best-fit model are given in Table 6. The model overestimates the peak temperature of a few transitions by $\sim 30-40 \%$ (at $87.236,96.062,96.120$, 104.793 , and $104.856 \mathrm{GHz}$ ) while all other transitions are well fitted; the reason for these discrepancies is unclear.

\subsection{Formamide $\left(\mathrm{NH}_{2} \mathrm{CHO}\right)$}

We use the CDMS entries for formamide in its ground and first vibrationally excited states (tags 45512 and 45516 , versions 2 and 1, respectively) and for its ${ }^{13} \mathrm{C}$ and ${ }^{15} \mathrm{~N}$ isotopologs in their ground state (tags 46512 and 46513, versions 2 and 1, respectively). These entries are based largely on Motiyenko et al. (2012), but also contain additional data. Laboratory data in the range of our survey were published by Kryvda et al. (2009). The entry for $v_{12}=1$ of the ${ }^{13} \mathrm{C}$ isotopolog was prepared by one of us (HSPM) based on data from Stubgaard (1978).

Formamide is well detected toward Sgr B2(N2), with 30 lines in its vibrational ground state and 13 in its vibrationally excited state $v_{12}=1$ (Figs. A.6 and A.7). Its ${ }^{13} \mathrm{C}$ isotopolog is also clearly detected, with 11 and 2 lines in its $v=0$ and $v_{12}=1$ states, respectively (Figs. A.8 and A.9). Finally, we report a tentative detection of ${ }^{15} \mathrm{NH}_{2} \mathrm{CHO}$, with one clearly detected line consistent with a ${ }^{14} \mathrm{~N} /{ }^{15} \mathrm{~N}$ isotopic ratio of 300 (Fig. A.10).

We derive a median emission size of $\sim 0.9-1.0^{\prime \prime}$ from Gaussian fits to the integrated intensity maps of the main and ${ }^{13} \mathrm{C}$ isotopologs, with a rms dispersion of $\sim 0.1$ " (see Figs. $3 \mathrm{c}$ and d). A number of lines of the main isotopolog are saturated and not well fitted by our simple LTE model. We selected only the transitions with an optical depth lower than 2 to build the population diagram shown in Fig. A.21. A fit to this diagram yields a rotational temperature of $155 \pm 3 \mathrm{~K}$ (Table 5). However, we obtain a rotational temperature of $200 \pm 14 \mathrm{~K}$ when we limit the fit to the transitions that belong to the vibrational ground state. The population diagram of the ${ }^{13} \mathrm{C}$ isotopolog is less populated (Fig. A.22) and yields a more uncertain rotational temperature of $222 \pm 35 \mathrm{~K}$ (Table 5). We adopt a temperature of $200 \mathrm{~K}$ for our LTE model of all isotopologs of formamide.

We initially modeled the spectra assuming a size of $0.9^{\prime \prime}$ as derived above but, in order to fit the $v_{12}=1$ transitions, we then had to assume a total column density of $\mathrm{NH}_{2} \mathrm{CHO}$ much lower than the one needed to fit the ground state transitions. By reducing the size to $0.8^{\prime \prime}$, we could attenuate the discrepancy between $v=0$ and $v_{12}=1$, however we still need a total column density 1.4 times lower to fit $v_{12}=1$. This is surprising because we do not face this problem for the ${ }^{13} \mathrm{C}$ isotopolog for which both states are well fitted assuming the same total column density. The discrepancy that affects the main isotopolog may be due to its higher optical depth although we do not feel that it is a satisfactory explanation.

\subsection{Isocyanic acid (HNCO)}

We use the CDMS entry for HNCO in its ground state (tag 43511 version 1) by Lapinov et al. (2007) with additional measurements in the range of our survey by Hocking et al. (1975), and the JPL entry for the ${ }^{13} \mathrm{C}$ isotopolog (tag 44008 version 1 , Hocking et al. 1975). Private entries for the vibrationally excited states $v_{5}=1, v_{6}=1$, and $v_{4}=1$ of the main isotopolog were prepared by one of us (HSPM). They are based on 
Table 6. Parameters of our best-fit LTE model of selected complex organic molecules toward Sgr B2(N2).

\begin{tabular}{|c|c|c|c|c|c|c|c|c|c|}
\hline Molecule & Status $^{a}$ & $N_{\operatorname{det}}^{b}$ & $\begin{array}{l}\operatorname{Size}^{c} \\
\left({ }^{\prime \prime}\right)\end{array}$ & $\begin{array}{c}T_{\text {rot }}{ }^{d} \\
(\mathrm{~K})\end{array}$ & $\begin{array}{c}N^{e} \\
\left(\mathrm{~cm}^{-2}\right)\end{array}$ & $F_{\mathrm{vib}}^{f}$ & $\begin{array}{c}\Delta V^{g} \\
\left(\mathrm{~km} \mathrm{~s}^{-1}\right)\end{array}$ & $\begin{array}{c}V_{\mathrm{off}^{h}} \\
\left(\mathrm{~km} \mathrm{~s}^{-1}\right)\end{array}$ & $\frac{N_{\mathrm{ref}}}{N} i$ \\
\hline $\mathrm{CH}_{3} \mathrm{NHCHO}, v_{\mathrm{t}}=0^{\star}$ & $\mathrm{t}$ & 4 & 0.9 & 180 & $1.0(17)$ & 1.26 & 5.0 & 0.5 & 1 \\
\hline$v_{\mathrm{t}}=1$ & $\mathrm{t}$ & 1 & 0.9 & 180 & $1.0(17)$ & 1.26 & 5.0 & 0.5 & 1 \\
\hline$v_{\mathrm{t}}=2$ & $\mathrm{t}$ & 0 & 0.9 & 180 & $1.0(17)$ & 1.26 & 5.0 & 0.5 & 1 \\
\hline $\mathrm{CH}_{3} \mathrm{NCO}, v_{\mathrm{b}}=0^{\star}$ & $\mathrm{d}$ & 60 & 1.2 & 150 & $2.2(17)$ & 1.00 & 5.0 & -0.6 & 1 \\
\hline$v_{\mathrm{b}}=1$ & $\mathrm{~d}$ & 4 & 1.2 & 150 & $2.2(17)$ & 1.00 & 5.0 & -0.6 & 1 \\
\hline $\mathrm{NH}_{2} \mathrm{CHO}, v=0^{\star}$ & $\mathrm{d}$ & 30 & 0.8 & 200 & $3.5(18)$ & 1.17 & 5.5 & 0.2 & 1 \\
\hline$v_{12}=1$ & $\mathrm{~d}$ & 13 & 0.8 & 200 & $2.6(18)$ & 1.17 & 5.5 & 0.2 & 1.4 \\
\hline $\mathrm{NH}_{2}{ }^{13} \mathrm{CHO}, v=0$ & $\mathrm{~d}$ & 11 & 0.8 & 200 & $1.3(17)$ & 1.17 & 5.5 & 0.5 & 27 \\
\hline$v_{12}=1$ & $\mathrm{~d}$ & 2 & 0.8 & 200 & $1.3(17)$ & 1.17 & 5.5 & 0.5 & 27 \\
\hline${ }^{15} \mathrm{NH}_{2} \mathrm{CHO}, v=0$ & $\mathrm{t}$ & 1 & 0.8 & 200 & $1.2(16)$ & 1.17 & 5.5 & 0.5 & 300 \\
\hline $\mathrm{HNCO}, v=0^{\star}$ & $\mathrm{d}$ & 12 & 0.9 & 240 & $2.0(18)$ & 1.06 & 5.5 & 0.0 & 1 \\
\hline$v_{5}=1$ & $\mathrm{~d}$ & 4 & 0.9 & 240 & $2.0(18)$ & 1.06 & 5.5 & 0.0 & 1 \\
\hline$v_{6}=1$ & $\mathrm{~d}$ & 1 & 0.9 & 240 & $2.0(18)$ & 1.06 & 5.5 & 0.0 & 1 \\
\hline$v_{4}=1$ & $\mathrm{t}$ & 0 & 0.9 & 240 & $2.0(18)$ & 1.06 & 5.5 & 0.0 & 1 \\
\hline $\mathrm{HN}^{13} \mathrm{CO}, v=0$ & $\mathrm{t}$ & 0 & 0.9 & 240 & $1.0(17)$ & 1.06 & 5.5 & 0.0 & 20 \\
\hline $\mathrm{CH}_{3} \mathrm{CONH}_{2}, v_{\mathrm{t}}=0^{\star}$ & $\mathrm{d}$ & 10 & 0.9 & 180 & $1.4(17)$ & 1.23 & 5.0 & 1.5 & 1 \\
\hline$v_{\mathrm{t}}=1$ & d & 8 & 0.9 & 180 & $1.4(17)$ & 1.23 & 5.0 & 1.5 & 1 \\
\hline$v_{\mathrm{t}}=2$ & d & 5 & 0.9 & 180 & $1.4(17)$ & 1.23 & 5.0 & 1.5 & 1 \\
\hline$\Delta v_{t} \neq 0$ & $\mathrm{t}$ & 0 & 0.9 & 180 & $1.4(17)$ & 1.23 & 5.0 & 1.5 & 1 \\
\hline
\end{tabular}

Notes. ${ }^{(a)} \mathrm{d}$ : detection, t: tentative detection. ${ }^{(b)}$ Number of detected lines (conservative estimate, see Sect. 3 of Belloche et al. 2016). One line of a given species may mean a group of transitions of that species that are blended together. ${ }^{(c)}$ Source diameter (FWHM). ${ }^{(d)}$ Rotational temperature. (e) Total column density of the molecule. $X(Y)$ means $X \times 10^{Y}$. An identical value for all listed vibrational/torsional states of a molecule means that LTE is an adequate description of the vibrational/torsional excitation. ${ }^{(f)}$ Correction factor that was applied to the column density to account for the contribution of vibrationally excited states, in the cases where this contribution was not included in the partition function of the spectroscopic predictions. ${ }^{(g)}$ Linewidth (FWHM). ${ }^{(h)}$ Velocity offset with respect to the assumed systemic velocity of Sgr B2(N2), $V_{\mathrm{lsr}}=74 \mathrm{~km} \mathrm{~s}{ }^{-1}$. ${ }^{(i)}$ Column density ratio, with $N_{\text {ref }}$ the column density of the previous reference species marked with a $\star$.

a preliminary, unpublished analysis of the ground and the four lowest excited states and were already used in Belloche et al. (2013). The data on the excited states was summarized in Niedenhoff et al. (1996). Transition frequencies in the range of our survey were published by Yamada \& Winnewisser (1977) and Yamada (1977).

Isocyanic acid is also well detected toward Sgr B2(N2), with twelves lines in its vibrational ground state, four in its vibrationally excited state $v_{5}=1$, and one in its state $v_{6}=1$ (Figs. A.11-A.13). Its state $v_{4}=1$, is not unambiguously detected but contributes significantly to the signal detected at 87.97 GHz so we have included it in our full model (Fig. A.14). The ${ }^{13} \mathrm{C}$ isotopolog is not unambiguously detected because all its significant transitions are located in the blueshifted wing of transitions of the main isotopolog, some of these suffering from absorption of the main isotopolog from the outer envelope of Sgr B2, which we have not yet taken into account in our full model (Fig. A.15). Nevertheless, the ${ }^{13} \mathrm{C}$ isotopolog contributes significantly to the signal detected at several frequencies, so we have included it in our full model, based on the model derived below for the main isotopolog and assuming a ${ }^{12} \mathrm{C} /{ }^{13} \mathrm{C}$ isotopic ratio of 20 .

We derive a median size of $0.9^{\prime \prime}$ from Gaussian fits to the integrated intensity maps of the main isotopolog, with a dispersion of $0.2^{\prime \prime}$ (see Fig. 3e). The population diagram shown in Fig. A.23 uses all but three transitions that are clearly detected, plus a number of additional lines that are more contaminated but for which we have identified and modeled the contaminating species. The three transitions of the vibrational ground state that we ignore (at 109.50, 109.91, and $110.30 \mathrm{GHz}$ ) have an opacity higher than 4. A fit to the population diagram yields a temperature of $220 \pm 10 \mathrm{~K}$. However, with this temperature, the optically thick lines of the ground state would saturate with a brightness temperature that is too low. As a compromise, we use a temperature of $240 \mathrm{~K}$.

The detected lines of all four states of the main isotopolog are relatively well reproduced with the same model parameters (Table 6). A few issues remain, however. First of all, the peak temperatures of the optically thick lines of $v=0$ are slightly underestimated, except for the peak temperature of the $5_{0,5}-4_{0,4}$ transition at $109.91 \mathrm{GHz}$ which is overestimated, probably because of spatial filtering of extended emission not taken into account in our model (see Fig. 6 of Jones et al. 2008). The second issue concerns two transitions with an upper energy level $E_{\text {up }} \sim 650 \mathrm{~K}, 34_{1,34}-35_{0,35}$ at $85.37 \mathrm{GHz}$ and $33_{1,33}-34_{0,34}$ at $109.96 \mathrm{GHz}$, which are both overestimated. The former is located in a frequency range affected by $\mathrm{c}-\mathrm{C}_{3} \mathrm{H}_{2}$ absorption by spiral arm clouds along the line of sight to Sgr B2, so the discrepancy at this frequency may not be a problem. We note, in addition, that the model for a third transition with $E_{\text {up }} \sim 650 \mathrm{~K}$, $5_{4,1}-4_{4,0}$ at $109.78 \mathrm{GHz}$, is consistent with the detected signal. Therefore, it is unclear why our model overestimates the peak temperature of the transition at $109.96 \mathrm{GHz}$.

\subsection{Acetamide $\left(\mathrm{CH}_{3} \mathrm{CONH}{ }_{2}\right)$}

We use predictions that are based on the measurements and analysis presented in Ilyushin et al. (2004) but were recomputed by 
one of us (V.V.I.) with the RAM36 code. We use the partition function calculated in Sect. 3 (Table 4).

With a total of 23 lines clearly detected in its ground state and its first and second torsionally excited states toward the hot core Sgr B2(N2), acetamide can be considered as securely detected (Figs. A.16-A.19). Fits to the integrated intensity maps of three of the detected lines give a median size of $\sim 1.0^{\prime \prime}$, with a dispersion of $0.2^{\prime \prime}$ (see Fig. 3f). The emission of four other detected lines is unresolved, pointing to a smaller size. The maps of the remaining detected lines were not fitted because they are, to some level, contaminated in their wings, which could bias the size measurements. A fit to the population diagram yields a rotational temperature of $226 \pm 33 \mathrm{~K}$, which is not well constrained (Fig. A.24 and Table 5). We adopt a temperature of $180 \mathrm{~K}$ and a size of $0.9^{\prime \prime}$ for our LTE model to make the comparison to $\mathrm{N}$-methylformamide more straightforward. The detected lines of the ground state $\left(v_{\mathrm{t}}=0\right)$ and both torsionally excited states $\left(v_{\mathrm{t}}=1\right.$ and 2$)$, as well as lines that connect different torsional states $\left(\Delta v_{\mathrm{t}} \neq 0\right)$, are well reproduced with the same model parameters (Table 6). One discrepancy can be noticed in Fig. A.16; the $v_{\mathrm{t}}=025_{20,6}-25_{18,7}$ transition of the $E$ species at $99.950 \mathrm{GHz}$ does not have a counterpart in the observed spectrum (at the $3 \sigma$ level). However it was extrapolated from a lower- $J$ fit $(J \leq 20)$ and has a frequency uncertainty of $200 \mathrm{kHz}$ which means that its frequency could be off by several times this number. Indeed, the actual positions of the hyperfine components of this line, as measured in the laboratory spectrum, are $99951.872 \mathrm{MHz}$ and $99952.331 \mathrm{MHz}$, both with an uncertainty of $10 \mathrm{kHz}$. At these frequencies, strong emission is detected toward Sgr B2(N2), which reconciles the spectrum expected for acetamide with the observed spectrum. Therefore, the discrepancy between the observed and synthetic spectra at 99.950 $\mathrm{GHz}$ does not pose a significant problem.

\section{Chemical modeling}

To investigate the production of the tentatively detected $\mathrm{N}$-methylformamide in Sgr B2(N2), we use the chemical kinetics model MAGICKAL (Garrod 2013) with an expanded gasgrain chemical network. This network is an extension of that presented by Belloche et al. (2014), and latterly by Müller et al. (2016a), and includes formation and destruction mechanisms for both $\mathrm{CH}_{3} \mathrm{NHCHO}$ and the related molecule $\mathrm{CH}_{3} \mathrm{NCO}$. The model allows for a treatment of the fully-coupled gas-phase, grain/ice-surface, and ice-mantle chemistry. The physical model follows that detailed in previous papers where a cold collapse phase to maximum density $\left(n_{\mathrm{H}}=2 \times 10^{8} \mathrm{~cm}^{-3}\right)$ and minimum dust-grain temperature $(8 \mathrm{~K})$ is followed by a warm-up from 8 to $400 \mathrm{~K}$; during this phase, the gas and dust temperatures are assumed to be well coupled. The initial chemical compositions used in the model follow those of Garrod (2013). The reader is referred to the above-mentioned publications for a more detailed discussion of the basic physical and chemical model. In the models presented here, we use the intermediate warm-up timescale, which generally produces the best match between models and observed abundances of other chemical species. The warm-up model therefore reaches a temperature of $200 \mathrm{~K}$ at $2 \times 10^{5} \mathrm{yr}$, reaching $400 \mathrm{~K}$ (and the end of the model run) at $\sim 2.85 \times 10^{5} \mathrm{yr}$.

The new network concentrates on the grain-surface production of the newly-introduced molecules. However, gas-phase destruction mechanisms for both molecules (as well as related intermediates) are included in the new network, the majority of which are ion-molecule processes or the subsequent dissociative recombination with electrons of the resultant molecular ions. Ion-molecule reactions are included for the major ionic species $\mathrm{C}^{+}, \mathrm{He}^{+}, \mathrm{H}_{3}{ }^{+}, \mathrm{H}_{3} \mathrm{O}^{+}$, and $\mathrm{HCO}^{+}$. Estimates for the rates of photo-dissociation of new molecules, as caused by cosmic ray-induced and (where extinction allows) external UV photons, are also included (see Garrod et al. 2008; Garrod 2013). Grain/ice-surface binding (desorption) energies for the new molecules are estimates based on interpolation/extrapolation of values for molecules with similar functional groups, following past publications. Binding energy values for $\mathrm{CH}_{3} \mathrm{NCO}$ and $\mathrm{CH}_{3} \mathrm{NHCHO}$ are $3575 \mathrm{~K}$ and $6281 \mathrm{~K}$, respectively, based on the formulations $\left[E_{\mathrm{des}}\left(\mathrm{CH}_{3}\right)+E_{\mathrm{des}}(\mathrm{N})+E_{\mathrm{des}}(\mathrm{C})+E_{\mathrm{des}}(\mathrm{O})\right]$ and $\left[E_{\mathrm{des}}\left(\mathrm{NH}_{2} \mathrm{CHO}\right)-E_{\mathrm{des}}(\mathrm{H})+E_{\mathrm{des}}\left(\mathrm{CH}_{3}\right)\right]$. We are not aware of any experimental determinations of these two quantities for appropriate surfaces.

In the new network, grain-surface and ice-mantle formation of $\mathrm{CH}_{3} \mathrm{NCO}$ occurs through a single radical-addition reaction:

$$
\mathrm{CH}_{3}+\mathrm{OCN} \rightarrow \mathrm{CH}_{3} \mathrm{NCO} \text {. }
$$

Each of the necessary radicals may be formed either through repetitive atomic addition or through the photo-dissociation of or chemical $\mathrm{H}$-abstraction from either $\mathrm{CH}_{4}$ or $\mathrm{HNCO}$.

To form $\mathrm{CH}_{3} \mathrm{NHCHO}$, a reaction involving the addition of a hydrogen atom to $\mathrm{CH}_{3} \mathrm{NCO}$ followed by the addition of another, presents itself as a possible route, that is,

$\mathrm{H}+\mathrm{CH}_{3} \mathrm{NCO} \rightarrow \mathrm{CH}_{3} \mathrm{NHCO}$

$\mathrm{H}+\mathrm{CH}_{3} \mathrm{NHCO} \rightarrow \mathrm{CH}_{3} \mathrm{NHCHO}$.

The first of these two reactions requires the breaking of a carbonnitrogen double bond, for which no activation energy barrier could be determined from the literature. The barrier to the similar reaction of $\mathrm{H}$ with $\mathrm{HNCO}$ has been determined experimentally in the gas phase by Nguyen et al. (1996) to be $1390 \mathrm{~K}$, although the value for $\mathrm{H}+\mathrm{CH}_{3} \mathrm{NCO}$ could plausibly be higher or lower. The expectation, however, is that, as with most barrier-mediated atomic-H reactions on cold grains, the mechanism would involve the tunneling of the $\mathrm{H}$ atom through the barrier, introducing further uncertainty into the reaction rate. By default, MAGICKAL uses a simple rectangular-barrier treatment to determine rates for tunneling reactions, typically assuming a uniform barrier width of $1 \AA$; the assumed height of the energy barrier therefore absorbs all other parameters pertaining to the overall reaction rate. The ideal activation energy barrier determined for the chemical model may therefore not be fully representative of the usual high-temperature value.

Because a broad range of activation energy values could be plausible for reaction (8), in the chemical network, we initially assume that the rate for reaction (8) is negligible (models M1M5), introducing non-zero values later.

Radical-addition reactions provide an alternative pathway to the formation of $\mathrm{N}$-methylformamide, through the reactions:

$\mathrm{CH}_{3}+\mathrm{HNCHO} \rightarrow \mathrm{CH}_{3} \mathrm{NHCHO}$
$\mathrm{HCO}+\mathrm{HNCH}_{3} \rightarrow \mathrm{CH}_{3} \mathrm{NHCHO}$.

The larger radicals in each of the above two reactions are produced through the addition of $\mathrm{NH}$ to either $\mathrm{CH}_{3}$ or $\mathrm{HCO}$. While cosmic ray-induced photo-dissociation of $\mathrm{CH}_{3} \mathrm{NH}_{2}$ and $\mathrm{NH}_{2} \mathrm{CHO}$ may also produce the necessary radicals, the chemical abstraction of a hydrogen atom by a radical from either molecule strongly favors the production of $\mathrm{CH}_{2} \mathrm{NH}_{2}$ or $\mathrm{NH}_{2} \mathrm{CO}$, respectively, rather than the alternative radicals that could play a part in forming $\mathrm{CH}_{3} \mathrm{NHCHO}$. However, in the case of $\mathrm{H}$-atom abstraction from $\mathrm{NH}_{2} \mathrm{CHO}$ by $\mathrm{OH}$, the model initially considers the 


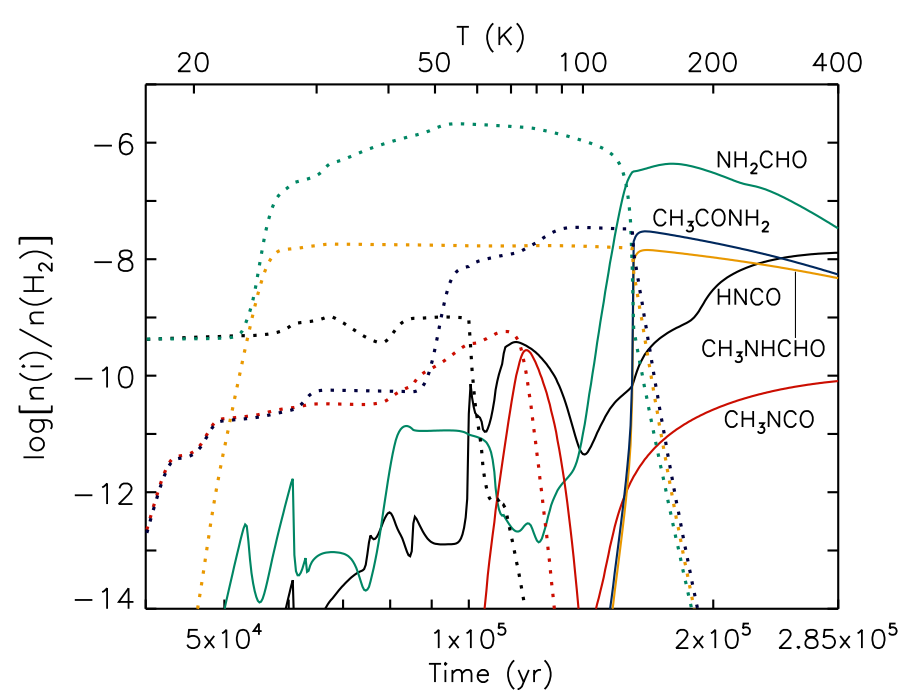

Fig. 5. Calculated abundances of selected chemical model species with respect to $\mathrm{H}_{2}$ for model M1, during warm-up from $8 \mathrm{~K}$ to $400 \mathrm{~K}$. Solid lines indicate gas-phase abundances; dotted lines of the same color indicate solid-phase abundances of the same species.

barriers to $\mathrm{NH}_{2} \mathrm{CO}$ and $\mathrm{NHCHO}$ production to be similar $(591 \mathrm{~K}$ versus $600 \mathrm{~K}$ ), following the estimates used by Garrod (2013), although neither value has been determined rigorously. Consequently, the influence of the production of HNCHO through this mechanism is also examined in Sect. 5.1.

\subsection{Model results}

Model M1 comprises the initial model in which conversion of $\mathrm{CH}_{3} \mathrm{NCO}$ to $\mathrm{CH}_{3} \mathrm{NHCHO}$ via consecutive $\mathrm{H}$ addition (reactions (8) and (9)) is switched off.

Figure 5 shows results from model M1. Time-dependent abundances for $\mathrm{CH}_{3} \mathrm{NCO}$ and $\mathrm{CH}_{3} \mathrm{NHCHO}$ as well as the related species $\mathrm{HNCO}, \mathrm{NH}_{2} \mathrm{CHO}$, and $\mathrm{CH}_{3} \mathrm{CONH}_{2}$ are shown. $\mathrm{CH}_{3} \mathrm{NHCHO}$ is seen to be formed on the grains (dotted line) in abundance at approximately $25 \mathrm{~K}$, coincident with a significant growth in $\mathrm{NH}_{2} \mathrm{CHO}$ production. Its formation is dominated by reaction (10). $\mathrm{CH}_{3} \mathrm{NCO}$ production on the grains is much more modest, and occurs only through reaction (7). Its gas-phase abundance peaks at a relatively low temperature, following its desorption from grains, and falls again, although it later begins to rise as the abundant $\mathrm{CH}_{3} \mathrm{NHCHO}$ is photodissociated in the gas phase. $\mathrm{CH}_{3} \mathrm{CONH}_{2}$ reaches a peak abundance just a little larger than that of $\mathrm{CH}_{3} \mathrm{NHCHO}$, forming mainly through the addition of $\mathrm{CH}_{3}$ and $\mathrm{NH}_{2} \mathrm{CO}$ radicals. $\mathrm{HNCO}$ is formed on the grains early $(T<20 \mathrm{~K})$ via hydrogenation of $\mathrm{OCN}$, which itself is formed through the atomic addition reactions $\mathrm{O}+\mathrm{CN}$ and $\mathrm{C}+$ NO. The gas-phase abundance of HNCO peaks at close to $60 \mathrm{~K}$ as it desorbs from the grains, then falls away. It is a significant by-product of the destruction of larger molecules at later times in the model, and its abundance continues to grow until the final model temperature of $400 \mathrm{~K}$ is reached.

Table 7 shows the peak gas-phase abundances of the plotted species, along with the temperatures at which those peaks are reached. It may be noted that the peak abundance of $\mathrm{CH}_{3} \mathrm{NHCHO}$ is approximately at parity with $\mathrm{HNCO}$, contrary to the observed column densities shown in Table 6 , where a ratio of $\sim 1: 20$ is obtained. The amount of $\mathrm{CH}_{3} \mathrm{NHCHO}$ produced in this model as compared with $\mathrm{CH}_{3} \mathrm{NCO}$ also appears high, while the abundance of $\mathrm{CH}_{3} \mathrm{NCO}$ is approximately one order of magnitude

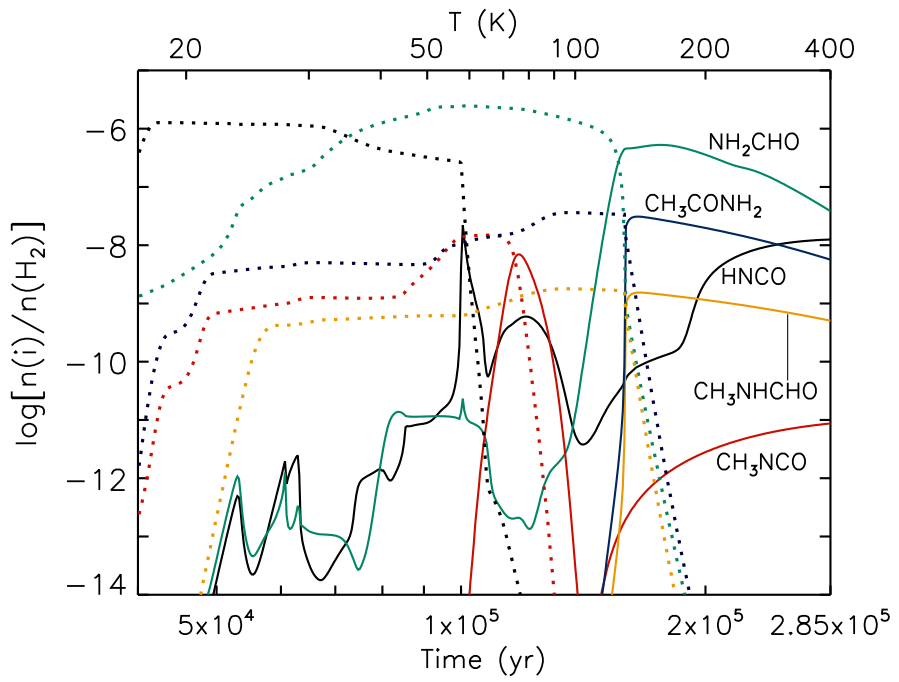

Fig. 6. As in Fig. 5 for model M4.

too low compared with HNCO. Since the sole production mechanism of $\mathrm{CH}_{3} \mathrm{NCO}$ relies on OCN, we have also considered alternative models, in which $\mathrm{HNCO}$, a possible precursor to OCN, may be more easily formed.

Models M2-M5, whose results are also tabulated in Table 7, allow the grain-surface reaction $\mathrm{NH}+\mathrm{CO} \rightarrow \mathrm{HNCO}$ to occur with a lower activation energy barrier than the $2500 \mathrm{~K}$ first assumed by Garrod et al. (2008), an estimate based loosely on the typically assumed barrier to the $\mathrm{H}$-addition reaction $\mathrm{H}+\mathrm{CO} \rightarrow$ HCO. These models show two types of behavior, with the threshold falling somewhere between models M2 and M3, that is, an activation energy, $E_{\mathrm{A}}$, between $2000 \mathrm{~K}$ and $1500 \mathrm{~K}$. Below this threshold, the models move away from M1-type behavior and instead show significantly increased HNCO production on the grains, such that the desorption of HNCO into the gas phase produces the peak abundance for this molecule. Figure 6 plots abundances for model M4, for which an activation energy barrier to the $\mathrm{NH}+\mathrm{CO}$ reaction of $1250 \mathrm{~K}$ is assumed. The peak abundance of $\mathrm{CH}_{3} \mathrm{NCO}$ is notably increased (see also Table 7) as a result of the greater $\mathrm{HNCO}$ abundance, which contributes to the production of OCN. Conversely, the abundance of $\mathrm{CH}_{3} \mathrm{NHCHO}$ is found to decrease markedly in models M3-M5 as the $\mathrm{NH}+\mathrm{CO}$ reaction becomes competitive with the $\mathrm{NH}+\mathrm{HCO} \rightarrow \mathrm{NHCHO}$ reaction at the key temperature $(\sim 25 \mathrm{~K})$ at which $\mathrm{CH}_{3}$ mobility makes reaction (10) important to $\mathrm{CH}_{3} \mathrm{NHCHO}$ production. Models M3-M5 all reach within a factor of $\sim 2$ of the observed $\mathrm{CH}_{3} \mathrm{NHCHO} / \mathrm{CH}_{3} \mathrm{NCO}$ abundance ratio.

To investigate the importance of $\mathrm{H}$-addition to $\mathrm{CH}_{3} \mathrm{NCO}$, we adjust the conditions assumed in model M4, taking a selection of activation energy barriers for reaction (8) to give a non-zero reaction rate. M4A assumes the same value as the reaction $\mathrm{H}+\mathrm{HNCO}$, while models M4B-E increase this value incrementally (see Table 8). We also switch off the $\mathrm{H}$-abstraction reaction $\mathrm{OH}+\mathrm{NH}_{2} \mathrm{CHO} \rightarrow \mathrm{NHCHO}$, to test its influence on $\mathrm{CH}_{3} \mathrm{NHCHO}$ production.

Model M4A demonstrates an extreme degree of conversion of $\mathrm{CH}_{3} \mathrm{NCO}$ to $\mathrm{CH}_{3} \mathrm{NHCHO}$ that is not borne out by the observations, while the somewhat higher barrier to hydrogenation of model M4B improves the match to the detected $\mathrm{CH}_{3} \mathrm{NHCHO} / \mathrm{CH}_{3} \mathrm{NCO}$ ratio (see also Table 9), albeit with a value greater than unity. Model M4C, as with M4, shows a modest dominance of $\mathrm{CH}_{3} \mathrm{NCO}$ over $\mathrm{CH}_{3} \mathrm{NHCHO}$, in line with observations, and is similar to M4 in the quality of its overall match 
A. Belloche et al.: Rotational spectroscopy, tentative interstellar detection, and chemical modeling of N-methylformamide

Table 7. Peak gas-phase fractional abundances with respect to $\mathrm{H}_{2}$ and temperatures at which the peak abundance values are achieved, for models with varying activation energy barriers for the grain-surface reaction $\mathrm{NH}+\mathrm{CO} \rightarrow \mathrm{HNCO}$.

\begin{tabular}{|c|c|c|c|c|c|c|c|c|c|c|}
\hline \multirow[b]{2}{*}{ Species } & \multicolumn{2}{|c|}{$\begin{array}{c}\mathrm{M} 1 \\
E_{\mathrm{A}}=2500 \mathrm{~K}\end{array}$} & \multicolumn{2}{|c|}{$\begin{array}{c}\mathrm{M} 2 \\
E_{\mathrm{A}}=2000 \mathrm{~K}\end{array}$} & \multicolumn{2}{|c|}{$\begin{array}{c}\mathrm{M} 3 \\
E_{\mathrm{A}}=1500 \mathrm{~K}\end{array}$} & \multicolumn{2}{|c|}{$\begin{array}{c}\text { M4 } \\
E_{\mathrm{A}}=1250 \mathrm{~K}\end{array}$} & \multicolumn{2}{|c|}{$\begin{array}{c}\text { M5 } \\
E_{\mathrm{A}}=1000 \mathrm{~K} \\
\end{array}$} \\
\hline & $n[\mathrm{i}] / n\left[\mathrm{H}_{2}\right]$ & $T(\mathrm{~K})$ & $n[\mathrm{i}] / n\left[\mathrm{H}_{2}\right]$ & $T(\mathrm{~K})$ & $n[\mathrm{i}] / n\left[\mathrm{H}_{2}\right]$ & $T(\mathrm{~K})$ & $n[\mathrm{i}] / n\left[\mathrm{H}_{2}\right]$ & $T(\mathrm{~K})$ & $n[\mathrm{i}] / n\left[\mathrm{H}_{2}\right]$ & $T(\mathrm{~K})$ \\
\hline $\mathrm{CH}_{3} \mathrm{NHCHO}$ & $1.4(-8)$ & 139 & $1.3(-8)$ & 139 & $1.6(-9)$ & 139 & $1.5(-9)$ & 139 & $1.5(-9)$ & 139 \\
\hline $\mathrm{CH}_{3} \mathrm{NCO}$ & $2.8(-10)$ & 74 & $1.6(-9)$ & 74 & $6.9(-9)$ & 74 & $6.9(-9)$ & 74 & $7.0(-9)$ & 75 \\
\hline $\mathrm{NH}_{2} \mathrm{CHO}$ & $4.3(-7)$ & 160 & $4.4(-7)$ & 160 & $5.3(-7)$ & 157 & $5.3(-7)$ & 157 & $5.4(-7)$ & 157 \\
\hline HNCO & $1.3(-8)$ & 398 & $1.3(-8)$ & 398 & $2.2(-8)$ & 56 & $2.2(-8)$ & 56 & $2.2(-8)$ & 56 \\
\hline $\mathrm{CH}_{3} \mathrm{CONH}_{2}$ & $3.0(-8)$ & 138 & $3.0(-8)$ & 138 & $3.1(-8)$ & 138 & $3.1(-8)$ & 138 & $3.2(-8)$ & 138 \\
\hline
\end{tabular}

Notes. Model M1 uses the value adopted in previous hot-core models. $X(Y)$ means $X \times 10^{Y}$.

Table 8. Peak gas-phase fractional abundances with respect to $\mathrm{H}_{2}$ and temperatures at which the peak abundance values are achieved, for models based on model M4, that is, with an activation energy barrier of $1250 \mathrm{~K}$ for the grain-surface reaction $\mathrm{NH}+\mathrm{CO} \rightarrow \mathrm{HNCO}$.

\begin{tabular}{|c|c|c|c|c|c|c|c|c|c|c|}
\hline \multirow[b]{2}{*}{ Species } & \multicolumn{2}{|c|}{$\begin{array}{c}\text { M4A } \\
E_{\mathrm{A}}=1390 \mathrm{~K}\end{array}$} & \multicolumn{2}{|c|}{$\begin{array}{c}\text { M4B } \\
E_{\mathrm{A}}=2000 \mathrm{~K}\end{array}$} & \multicolumn{2}{|c|}{$\begin{array}{c}\mathrm{M} 4 \mathrm{C} \\
E_{\mathrm{A}}=2500 \mathrm{~K}\end{array}$} & \multicolumn{2}{|c|}{$\begin{array}{c}\text { M4D } \\
E_{\mathrm{A}}=3000 \mathrm{~K}\end{array}$} & \multicolumn{2}{|c|}{$\begin{array}{c}\text { M4E } \\
E_{\mathrm{A}}=3500 \mathrm{~K} \\
\end{array}$} \\
\hline & $n[\mathrm{i}] / n\left[\mathrm{H}_{2}\right]$ & $T(\mathrm{~K})$ & $n[\mathrm{i}] / n\left[\mathrm{H}_{2}\right]$ & $T(\mathrm{~K})$ & $n[\mathrm{i}] / n\left[\mathrm{H}_{2}\right]$ & $T(\mathrm{~K})$ & $n[\mathrm{i}] / n\left[\mathrm{H}_{2}\right]$ & $T(\mathrm{~K})$ & $n[\mathrm{i}] / n\left[\mathrm{H}_{2}\right]$ & $T(\mathrm{~K})$ \\
\hline $\mathrm{CH}_{3} \mathrm{NHCHO}$ & $1.2(-8)$ & 139 & $5.3(-9)$ & 139 & $1.2(-9)$ & 139 & $6.0(-10)$ & 139 & $5.1(-10)$ & 139 \\
\hline $\mathrm{CH}_{3} \mathrm{NCO}$ & $7.0(-11)$ & 398 & $4.1(-9)$ & 74 & $6.5(-9)$ & 74 & $6.9(-9)$ & 74 & $6.9(-9)$ & 74 \\
\hline $\mathrm{NH}_{2} \mathrm{CHO}$ & $5.1(-7)$ & 157 & $5.1(-7)$ & 157 & $5.1(-7)$ & 157 & $5.1(-7)$ & 157 & $5.1(-7)$ & 157 \\
\hline HNCO & $2.2(-8)$ & 56 & $2.2(-8)$ & 56 & $2.2(-8)$ & 56 & $2.2(-8)$ & 56 & $2.2(-8)$ & 56 \\
\hline $\mathrm{CH}_{3} \mathrm{CONH}_{2}$ & $3.2(-8)$ & 138 & $3.2(-8)$ & 138 & $3.2(-8)$ & 138 & $3.2(-8)$ & 138 & $3.2(-8)$ & 138 \\
\hline
\end{tabular}

Notes. The models (M4A-E) vary from model M4 in two ways: (i) the $\mathrm{OH}+\mathrm{NH}_{2} \mathrm{CHO} \rightarrow \mathrm{NHCHO}+\mathrm{H}_{2} \mathrm{O}$ reaction pathway has been switched off; (ii) the reaction $\mathrm{H}+\mathrm{CH}_{3} \mathrm{NCO} \rightarrow \mathrm{CH}_{3} \mathrm{NHCO}$ is switched on, with an activation energy barrier, $E_{\mathrm{A}}$, as indicated. Model M4A uses the value assumed for the $\mathrm{H}+\mathrm{HNCO} \rightarrow \mathrm{NH}_{2} \mathrm{CO}$ reaction (1390 K, Nguyen et al. 1996), while the other models use larger values. $X(Y)$ means $X \times 10^{Y}$.

with observations. At the higher activation energies used in models M4D and M4E, the importance of reaction (8) diminishes. Here, the influence of the removal of the $\mathrm{OH}+\mathrm{NH}_{2} \mathrm{CHO} \rightarrow$ $\mathrm{NHCHO}$ reaction becomes apparent via comparison with model M4 (Table 7); the abundance of $\mathrm{CH}_{3} \mathrm{NHCHO}$ falls by twothirds. For each of the models M4A-E, the behavior of HNCO, $\mathrm{NH}_{2} \mathrm{CHO}$, and $\mathrm{CH}_{3} \mathrm{CONH}_{2}$ are little affected in comparison to model M4.

Note that particularly in the cases of $\mathrm{CH}_{3} \mathrm{NCO}$ and $\mathrm{CH}_{3} \mathrm{CONH}_{2}$, the temperatures at which peak abundances are attained are somewhat lower than the rotational temperatures obtained from the spectroscopic model fits to the observational data. This may be due to the imprecision of either or both of the binding energy estimates and the spectroscopic fits. The observed HNCO temperature is best represented by the latetime/high-temperature peak found in the models, rather than the brief gas-phase spike at around $60 \mathrm{~K}$. The precise temperature at which the high-temperature peak is reached is dependent on the destruction rates and warm-up timescale assumed in the model. In the case of $\mathrm{CH}_{3} \mathrm{NCO}$, the larger spatial extent of this molecule (1.2"), as derived from the observations, compared with that of $\mathrm{CH}_{3} \mathrm{NHCHO}$ (0.9") is consistent with the idea that the former is released from grains at lower temperatures than the latter.

Table 9 shows ratios of the abundances of the main molecules to $\mathrm{HNCO}$, as well as the $\mathrm{CH}_{3} \mathrm{NHCHO} / \mathrm{CH}_{3} \mathrm{NCO}$ and $\mathrm{CH}_{3} \mathrm{NHCHO} / \mathrm{CH}_{3} \mathrm{CONH}_{2}$ ratios. Values are shown for the initial model M1, as well as representative models from Tables 7 and 8 that show a good match with observed values. Models M4 and M4C both show similarly good agreement with observed ratios involving $\mathrm{CH}_{3} \mathrm{NCO}$ and $\mathrm{CH}_{3} \mathrm{NHCHO}$, while M4B is significantly further from the observed $\mathrm{CH}_{3} \mathrm{NHCHO} / \mathrm{HNCO}$ ratio. However, all four models produce an overabundance of $\mathrm{NH}_{2} \mathrm{CHO}$ and $\mathrm{CH}_{3} \mathrm{CONH}_{2}$ by more than an order of magnitude.
Because acetamide, $\mathrm{CH}_{3} \mathrm{CONH}_{2}$, is predominantly formed as a result of $\mathrm{H}$-abstraction from formamide, followed by methylgroup addition, its overabundance is related to that of $\mathrm{NH}_{2} \mathrm{CHO}$. The majority of the latter molecule is formed via the reaction $\mathrm{NH}_{2}+\mathrm{H}_{2} \mathrm{CO} \rightarrow \mathrm{NH}_{2} \mathrm{CHO}+\mathrm{H}$, which we include on the grains as well as in the gas phase, assuming the activation energy barrier determined by Barone et al. (2015) of 26.9 K. However, Song \& Kästner (2016) find a substantially higher barrier that would render the reaction rate negligible (as determined for a gas-phase interaction). In order to test this possibility, we remove the $\mathrm{NH}_{2}+\mathrm{H}_{2} \mathrm{CO}$ mechanism both in the gas phase and on grains. This reduces $\mathrm{NH}_{2} \mathrm{CHO}$ production by approximately an order of magnitude and brings the peak $\mathrm{NH}_{2} \mathrm{CHO}$ : $\mathrm{HNCO}$ ratio achieved in the models to a very good match with the observed value (1.2 versus 1.8). The removal of either the gas-phase or grain-surface mechanism alone is not sufficient to reduce $\mathrm{NH}_{2} \mathrm{CHO}$ abundance, as both contribute significantly in the present implementation. However, even the better match to observed $\mathrm{NH}_{2} \mathrm{CHO}$ is not sufficient to bring down acetamide abundances to appropriate levels, as the alternative $\mathrm{NH}_{2}+\mathrm{CH}_{3} \mathrm{CO}$ formation mechanism is also important to its formation. The production of acetamide in hot cores merits further detailed study.

While models M4 and M4C show similar agreement with observations in spite of the different dominant chemical pathways involved for $\mathrm{CH}_{3} \mathrm{NHCHO}$ production, the fact that $\mathrm{NH}_{2} \mathrm{CO}$ is a more likely product of the reaction between $\mathrm{OH}$ and $\mathrm{NH}_{2} \mathrm{CHO}$ than NHCHO (judging by typical barriers to $\mathrm{H}$-abstraction from an amino versus a carbonyl group) makes model $\mathrm{M} 4 \mathrm{C}$ more plausible in this respect. The observed $\mathrm{CH}_{3} \mathrm{NHCHO} / \mathrm{CH}_{3} \mathrm{NCO}$ ratio is bracketed by the values obtained with models M4B and $\mathrm{M} 4 \mathrm{C}$, indicating indeed that a mechanism of direct hydrogenation from $\mathrm{CH}_{3} \mathrm{NCO}$ to $\mathrm{CH}_{3} \mathrm{NHCHO}$ is capable of reproducing observations of these molecules. However, given an 
Table 9. Relative abundances for a selection of chemical species, from the initial model (M1) and the three other most successful models, and from the observations toward Sgr B2(N2)

\begin{tabular}{|c|c|c|c|c|c|}
\hline Species & M1 & M4 & M4B & M4C & Observations \\
\hline $\mathrm{CH}_{3} \mathrm{NHCHO} / \mathrm{HNCO}$ & 1.1 & 0.070 & 0.24 & 0.055 & 0.050 \\
\hline $\mathrm{CH}_{3} \mathrm{NCO} / \mathrm{HNCO}$ & 0.021 & 0.32 & 0.19 & 0.30 & 0.11 \\
\hline $\mathrm{NH}_{2} \mathrm{CHO} / \mathrm{HNCO}$ & 34 & 24 & 23 & 23 & 1.8 \\
\hline $\mathrm{CH}_{3} \mathrm{CONH}_{2} / \mathrm{HNCO}$ & 2.3 & 1.4 & 1.5 & 1.5 & 0.070 \\
\hline $\mathrm{CH}_{3} \mathrm{NHCHO} / \mathrm{CH}_{3} \mathrm{NCO}$ & 52 & 0.22 & 1.3 & 0.18 & 0.45 \\
\hline 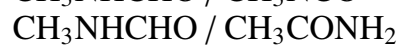 & 0.48 & 0.050 & 0.17 & 0.037 & 0.71 \\
\hline
\end{tabular}

appropriate activation energy barrier, both reactions (8) and (10) may produce sufficient quantities of $\mathrm{CH}_{3} \mathrm{NHCHO}$ to agree reasonably with observations.

In order to achieve observed abundances of $\mathrm{CH}_{3} \mathrm{NCO}$, a barrier to the reaction $\mathrm{NH}+\mathrm{CO} \rightarrow \mathrm{HNCO}$ of no more than $\sim 1500 \mathrm{~K}$ is required, allowing this to become the dominant mechanism by which HNCO is formed on grains (although its abundance in the gas phase is still dominated by its formation as a by-product of the destruction of larger species). Under such conditions, a barrier to the hydrogenation of $\mathrm{CH}_{3} \mathrm{NCO}$ may be estimated to be approximately $2000 \mathrm{~K}$ to best reproduce observed abundances of N-methylformamide.

\section{Discussion}

The spectroscopic results obtained in Sect. 3 represent a significant improvement in the characterization of the spectrum of N-methylformamide. First of all, we have substantially expanded the frequency coverage. Second, the rotational lines belonging to the first and second excited torsional states of $\mathrm{N}$-methylformamide have been assigned and fitted for the first time. Finally, the resulting best model is capable of reproducing the assigned data set within experimental error, including the lines from previous studies that were excluded from the fits due to large observed-minus-calculated values (Kawashima et al. 2010).

All the chemical models computed in the course of this work predict a substantial abundance of $\mathrm{CH}_{3} \mathrm{NHCHO}$ compared to $\mathrm{CH}_{3} \mathrm{NCO}$. The smallest ratios were produced by models M4D and $\mathrm{M} 4 \mathrm{E}$, with $\left[\mathrm{CH}_{3} \mathrm{NHCHO}\right] /\left[\mathrm{CH}_{3} \mathrm{NCO}\right] \sim 0.07-0.09$, but all other models have $\left[\mathrm{CH}_{3} \mathrm{NHCHO}\right] /\left[\mathrm{CH}_{3} \mathrm{NCO}\right] \gtrsim 0.2$. Two of the chemical models provide values that bracket the observed ratio, thus a fine tuning of the barrier against hydrogenation of $\mathrm{CH}_{3} \mathrm{NCO}$ would allow the observed ratio to be achieved. The chemical modeling thus gives some additional support to the tentative interstellar detection of $\mathrm{CH}_{3} \mathrm{NHCHO}$.

$\mathrm{N}$-methylformamide, $\mathrm{CH}_{3} \mathrm{NHCHO}$, is a structural isomer of acetamide, $\mathrm{CH}_{3} \mathrm{CONH}_{2}$. As mentioned in Sect. $1, \mathrm{CH}_{3} \mathrm{NHCHO}$ is the second most stable $\mathrm{C}_{2} \mathrm{H}_{5} \mathrm{NO}$ isomer, $\mathrm{CH}_{3} \mathrm{CONH}_{2}$ being the most stable one (Lattelais et al. 2010). In Sect. 4, we found that, provided its detection is confirmed, N-methylformamide is slightly less abundant than acetamide in Sgr B2(N2), which, at first sight, appears to be in agreement with the minimum energy principle initially put forward by Lattelais et al. (2009). This principle states that the most abundant isomer of a given generic chemical formula should be the most stable one thermodynamically. Lattelais et al. (2009) found a correlation between the observed abundance ratios of isomers of several generic chemical formulae as a function of their zero-point energy difference. According to this relation, N-methylformamide should be approximately 3.5 times less abundant than acetamide while it is only a factor 1.4 less abundant in Sgr B2(N2). The discrepancy is only slightly larger than a factor of two, but it tends to suggest that $\mathrm{N}$-methylformamide does not follow this correlation closely. In addition, the range of kinetic parameters explored in the chemical models presented here produce variations in the abundance of $\mathrm{CH}_{3} \mathrm{NHCHO}$ of more than one order of magnitude. In the case of acetamide, while the models all produce an excess over the abundance of $\mathrm{N}$-methylformamide, variations in other model parameters within a plausible range can produce variations in $\mathrm{CH}_{3} \mathrm{CONH}_{2}$ abundance that are comparable with those of $\mathrm{CH}_{3} \mathrm{NHCHO}$. The availablility of key precursor radicals at the optimal temperature for diffusion is one of the key influences on the production of such molecules, and one that is unlikely to be controlled purely by the thermodynamic properties of the products. Therefore, the abundance ratio of the two isomers has most likely an origin based on kinetics, rather than a simple thermal equilibrium. A similar conclusion was obtained by Loomis et al. (2015) and Loison et al. (2016) based on observations of the isomers of $\mathrm{C}_{3} \mathrm{H}_{2} \mathrm{O}$, whose abundance ratios are not consistent with the minimum energy principle.

A further test would be to measure the abundance of the next $\mathrm{C}_{2} \mathrm{H}_{5} \mathrm{NO}$ isomer, acetimidic acid, $\mathrm{CH}_{3} \mathrm{C}(\mathrm{OH}) \mathrm{NH}$, which has a slightly higher zero-point energy than $\mathrm{N}$-methylformamide (Lattelais et al. 2010). This would require laboratory measurements to characterize the rotational spectrum of this molecule and produce spectroscopic predictions suitable for an astronomical search. The dipole moment of this molecule, however, is more than a factor of 2 smaller than the ones of acetamide and $\mathrm{N}$-methylformamide (Lattelais et al. 2009), making its detection more challenging.

\section{Conclusions}

The rotational spectrum of the trans conformer of $\mathrm{N}$-methylformamide was studied in the laboratory in the frequency range from 45 to $630 \mathrm{GHz}$ using two different spectrometers in Kharkiv and Lille. The new data provides significant expansion both in frequency range (from $118 \mathrm{GHz}$ to $630 \mathrm{GHz}$ ) and quantum number coverage (from $J=11$ to $J=62$ ), including the first assignment of the rotational spectra of the first and second excited torsional states of N-methylformamide. The final data set contains $12456 A$ - and $E$-type transitions in the ground, first, and second excited torsional states of the trans conformer. Our theoretical model fits the available data with a weighted root-mean-square deviation of 0.84 , that is, within experimental error. The obtained results provide a firm basis for reliable predictions of the N-methylformamide spectrum in the millimeter and submillimeter wavelength range for the needs of radio astronomy. 
Using the spectroscopic predictions obtained here, we report the first tentative interstellar detection of $\mathrm{N}$-methylformamide. The main results of this study can be summarized as follows:

1. Five transitions of $\mathrm{N}$-methylformamide are coincident with spectral lines detected toward the hot molecular core Sgr B2(N2). These lines are not contaminated by other species, and their intensities are well reproduced by our LTE model of N-methylformamide. This suggests that the molecule may be present in this source.

2. We derive a column density of $\sim 1 \times 10^{17} \mathrm{~cm}^{-2}$ for $\mathrm{N}$-methylformamide. The molecule is more than one order of magnitude less abundant than formamide, twice less abundant than methyl isocyanate, and only slightly less abundant than acetamide.

3. Our gas-grain chemical kinetics model is able to reproduce the abundance ratio of $\mathrm{N}$-methylformamide to methyl isocyanate using kinetic parameters within a plausible range, supporting the tentative detection of the former.

4. The chemical models indicate that the efficient formation of $\mathrm{HNCO}$ via $\mathrm{NH}+\mathrm{CO}$ on grains is a necessary step in the achievement of the observed gas-phase abundance of $\mathrm{CH}_{3} \mathrm{NCO}$

5. Production of $\mathrm{CH}_{3} \mathrm{NHCHO}$ may plausibly occur on grains either through the direct addition of functional-group radicals or through the hydrogenation of $\mathrm{CH}_{3} \mathrm{NCO}$.

6. We also report the tentative detection of the ${ }^{15} \mathrm{~N}$ isotopolog of formamide toward Sgr B2(N2) with a ${ }^{14} \mathrm{~N} /{ }^{15} \mathrm{~N}$ isotopic ratio of 300 .

Provided the detection of $\mathrm{N}$-methylformamide is confirmed, the only slight underabundance of this molecule compared to its more stable structural isomer and the sensitivity of the model abundances to the variations of the model parameters suggest that the formation of these two molecules is controlled by kinetics rather than thermal equilibrium. The interstellar detection of the next stable isomer of the $\mathrm{C}_{2} \mathrm{H}_{5} \mathrm{NO}$ family, $\mathrm{CH}_{3} \mathrm{C}(\mathrm{OH}) \mathrm{NH}$, may therefore become possible once its rotational spectrum has been measured in the laboratory.

Acknowledgements. This paper makes use of the following ALMA data ADS/JAO.ALMA\#2011.0.00017.S, ADS/JAO.ALMA\#2012.1.00012.S. ALMA is a partnership of ESO (representing its member states), NSF (USA), and NINS (Japan), together with NRC (Canada), NSC and ASIAA (Taiwan), and KAS (Republic of Korea), in cooperation with the Republic of Chile. The Joint ALMA Observatory is operated by ESO, AUI/NRAO, and NAOJ. The interferometric data are available in the ALMA archive at https://almascience.eso.org/ aq/. Part of this work was done within the Ukrainian-French CNRS-PICS \# 6051 project. This work has been in part supported by the Deutsche Forschungsgemeinschaft (DFG) through the collaborative research grant SFB 956 "Conditions and Impact of Star Formation", project area B3. Our research benefited from NASA's Astrophysics Data System (ADS)

\section{References}

Alekseev, E., Motiyenko, R., \& Margulès, L. 2012, Radio Physics and Radio Astronomy, 3, 75

Barone, V., Latouche, C., Skouteris, D., et al. 2015, MNRAS, 453, L31
Belloche, A., Müller, H. S. P., Menten, K. M., Schilke, P., \& Comito, C. 2013, A\&A, 559, A47

Belloche, A., Garrod, R. T., Müller, H. S. P., \& Menten, K. M. 2014, Science, 345, 1584

Belloche, A., Müller, H. S. P., Garrod, R. T., \& Menten, K. M. 2016, A\&A, 587, A91

Cernicharo, J., Kisiel, Z., Tercero, B., et al. 2016, A\&A, 587, L4

Fantoni, A., \& Caminati, W. 1996, J. Chem. Soc., Faraday Trans., 92, 343

Fantoni, A. C., Caminati, W., Hartwig, H., \& Stahl, W. 2002, J. Mol. Struct., 612, 305

Garrod, R. T. 2013, ApJ, 765, 60

Garrod, R. T., Widicus Weaver, S. L., \& Herbst, E. 2008, ApJ, 682, 283

Goesmann, F., Rosenbauer, H., Bredehöft, J. H., et al. 2015, Science, 349, aab0689

Gordy, W., \& Cook, R. L. 1984, Microwave Molecular Spectra, Techniques of Chemistry, Vol. XVIII (New York: Wiley)

Halfen, D. T., Ilyushin, V., \& Ziurys, L. M. 2011, ApJ, 743, 60

Halfen, D. T., Ilyushin, V. V., \& Ziurys, L. M. 2015, ApJ, 812, L5

Hirota, E., Kawashima, Y., Usami, T., \& Seto, K. 2010, J. Mol. Spectr., 260, 30

Hocking, W. H., Gerry, M. C. L., \& Winnewisser, G. 1975, Can. J. Phys., 53, 1869

Hollis, J., Lovas, F. J., Remijan, A. J., et al. 2006, ApJ, 643, L25

Hougen, J. T., Kleiner, I., \& Godefroid, M. 1994, J. Mol. Spectr., 163, 559

Ilyushin, V. V., Alekseev, E. A., Dyubko, S. F., Kleiner, I., \& Hougen, J. T. 2004, J. Mol. Spectr., 227, 115

Ilyushin, V. V., Kisiel, Z., Pszczólkowski, L., Mäder, H., \& Hougen, J. T. 2010 J. Mol. Spectr., 259, 26

Ilyushin, V. V., Endres, C. P., Lewen, F., Schlemmer, S., \& Drouin, B. J. 2013, J. Mol. Spectr., 290, 31

Jones, P. A., Burton, M. G., Cunningham, M. R., et al. 2008, MNRAS, 386, 117 Kaiser, R., Stockton, A., Kim, Y., Jensen, E., \& Mathies, R. 2013, ApJ, 765, 111 Kawashima, Y., Usami, T., Suenram, R. D., Golubiatnikov, G. Y., \& Hirota, E. 2010, J. Mol. Spectr., 263, 11

Kleiner, I. 2010, J. Mol. Spectr., 260, 1

Koput, J. 1986, J. Mol. Spectr., 115, 131

Kryvda, A. V., Gerasimov, V. G., Dyubko, S. F., Alekseev, E. A., \& Motiyenko, R. A. 2009, J. Mol. Spectr., 254, 28

Kutzelnigg, W., \& Mecke, R. 1962, Spectrochim. Acta, 18, 549

Kydd, R. A., \& Dunham, A. R. C. 1980, J. Mol. Struct., 69, 79

Lapinov, A. V., Golubiatnikov, G. Y., Markov, V. N., \& Guarnieri, A. 2007, Astron. Lett., 33, 121

Lattelais, M., Pauzat, F., Ellinger, Y., \& Ceccarelli, C. 2009, ApJ, 696, L133

Lattelais, M., Pauzat, F., Ellinger, Y., \& Ceccarelli, C. 2010, A\&A, 519, A30

Lin, C. C., \& Swalen, J. D. 1959, Rev. Mod. Phys., 31, 841

Loison, J.-C., Agúndez, M., Marcelino, N., et al. 2016, MNRAS, 456, 4101

Loomis, R. A., McGuire, B. A., Shingledecker, C., et al. 2015, ApJ, 799, 34

Margulès, L., Belloche, A., Müller, H. S. P., et al. 2016, A\&A, 590, A93

Motiyenko, R., Tercero, B., Cernicharo, J., \& Margulès, L. 2012, A\&A, 548, A71

Müller, H. S. P., Schlöder, F., Stutzki, J., \& Winnewisser, G. 2005, J. Mol. Struct., 742,215

Müller, H. S. P., Belloche, A., Xu, L.-H., et al. 2016a, A\&A, 587, A92

Müller, H. S. P., Walters, A., Wehres, N., et al. 2016b, A\&A, 595, A87

Nguyen, M. T., Sengupta, D., Vereecken, L., Peeters, J., \& Vanquickenborne, L. G. 1996, J. Phys. Chem., 100, 1615

Niedenhoff, M., Yamada, K. M. T., \& Winnewisser, G. 1996, J. Mol. Spectr., 176,342

Rubin, R., Swenson Jr, G., Benson, R., Tigelaar, H., \& Flygare, W. 1971, ApJ, 169, L39

Song, L., \& Kästner, J. 2016, Phys. Chem. Chem. Phys., 18, 29278

Stubgaard, M. 1978, Ph.D. Thesis, Københavns Universitet, Denmark

Xu, L.-H., Fisher, J., Lees, R., et al. 2008, J. Mol. Spectr., 251, 305

Yamada, K. 1977, J. Mol. Spectr., 68, 423

Yamada, K., \& Winnewisser, M. 1977, J. Mol. Spectr., 68, 307

Zakharenko, O., Motiyenko, R. A., Margulès, L., \& Huet, T. R. 2015, J. Mol. Spectr., 317, 41 


\section{Appendix A: Complementary figures}

Figures A.1-A.19 show the transitions of $\mathrm{CH}_{3} \mathrm{NHCHO}$, $\mathrm{CH}_{3} \mathrm{NCO}, \mathrm{NH}_{2} \mathrm{CHO}, \mathrm{HNCO}, \mathrm{CH}_{3} \mathrm{CONH}_{2}$, and some of their isotopologs or vibrationally excited states that are covered by the EMoCA survey and contribute significantly to the signal detected toward Sgr B2(N2). Figures A.20-A.24 show the population diagrams of these molecules except the one of $\mathrm{CH}_{3} \mathrm{NHCHO}$, which is shown in Fig. 4.
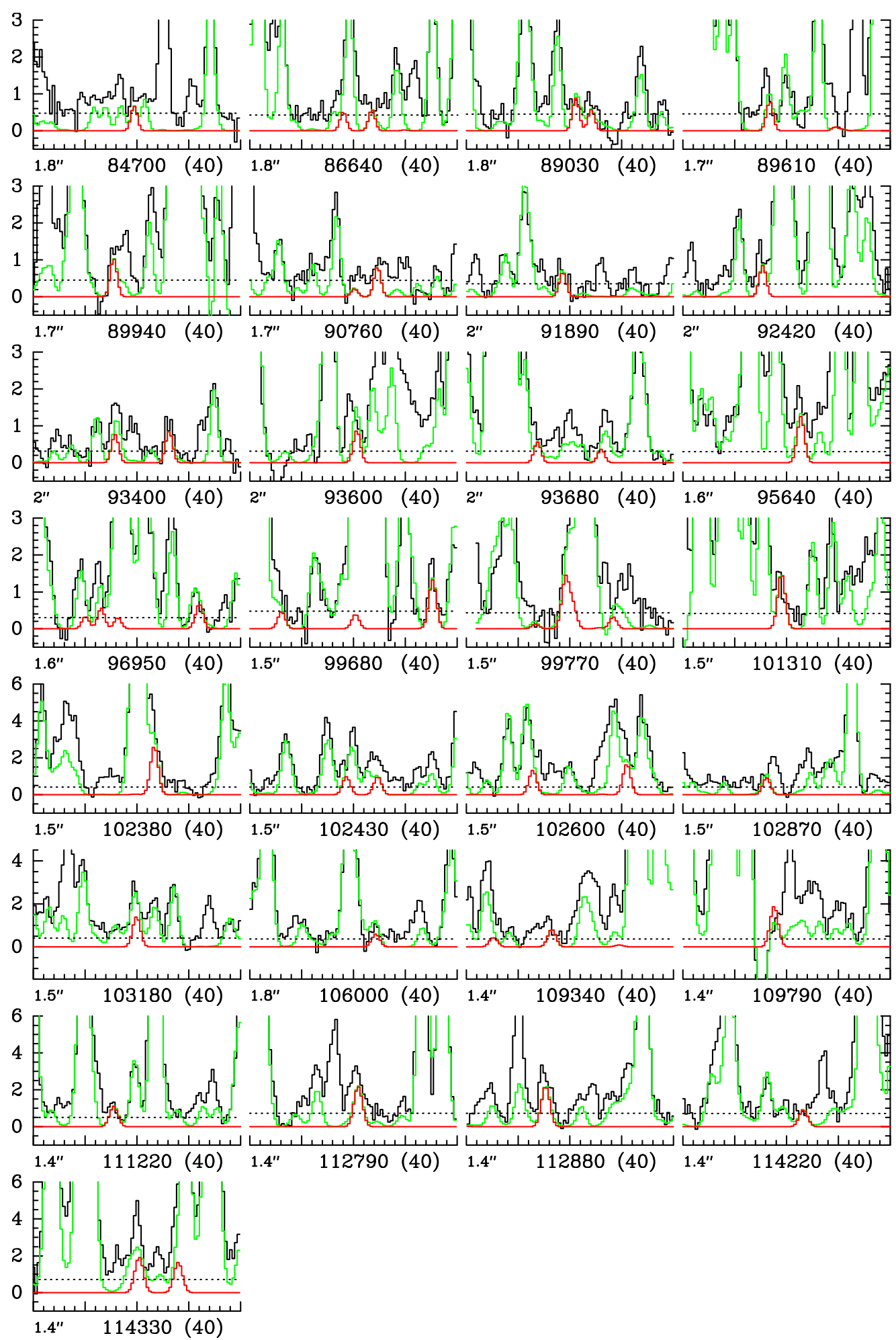

Fig. A.1. Transitions of $\mathrm{CH}_{3} \mathrm{NHCHO}, v_{\mathrm{t}}=0$ covered by our ALMA survey. The best-fit LTE synthetic spectrum of $\mathrm{CH}_{3} \mathrm{NHCHO}, v_{\mathrm{t}}=0$ is displayed in red and overlaid on the observed spectrum of Sgr B2(N2) shown in black. The green synthetic spectrum contains the contributions of all molecules identified in our survey so far, including the species shown in red. The central frequency and width are indicated in $\mathrm{MHz}$ below each panel. The angular resolution (HPBW) is also indicated. The $y$-axis is labeled in brightness temperature units (K). The dotted line indicates the $3 \sigma$ noise level. 
A. Belloche et al.: Rotational spectroscopy, tentative interstellar detection, and chemical modeling of N-methylformamide

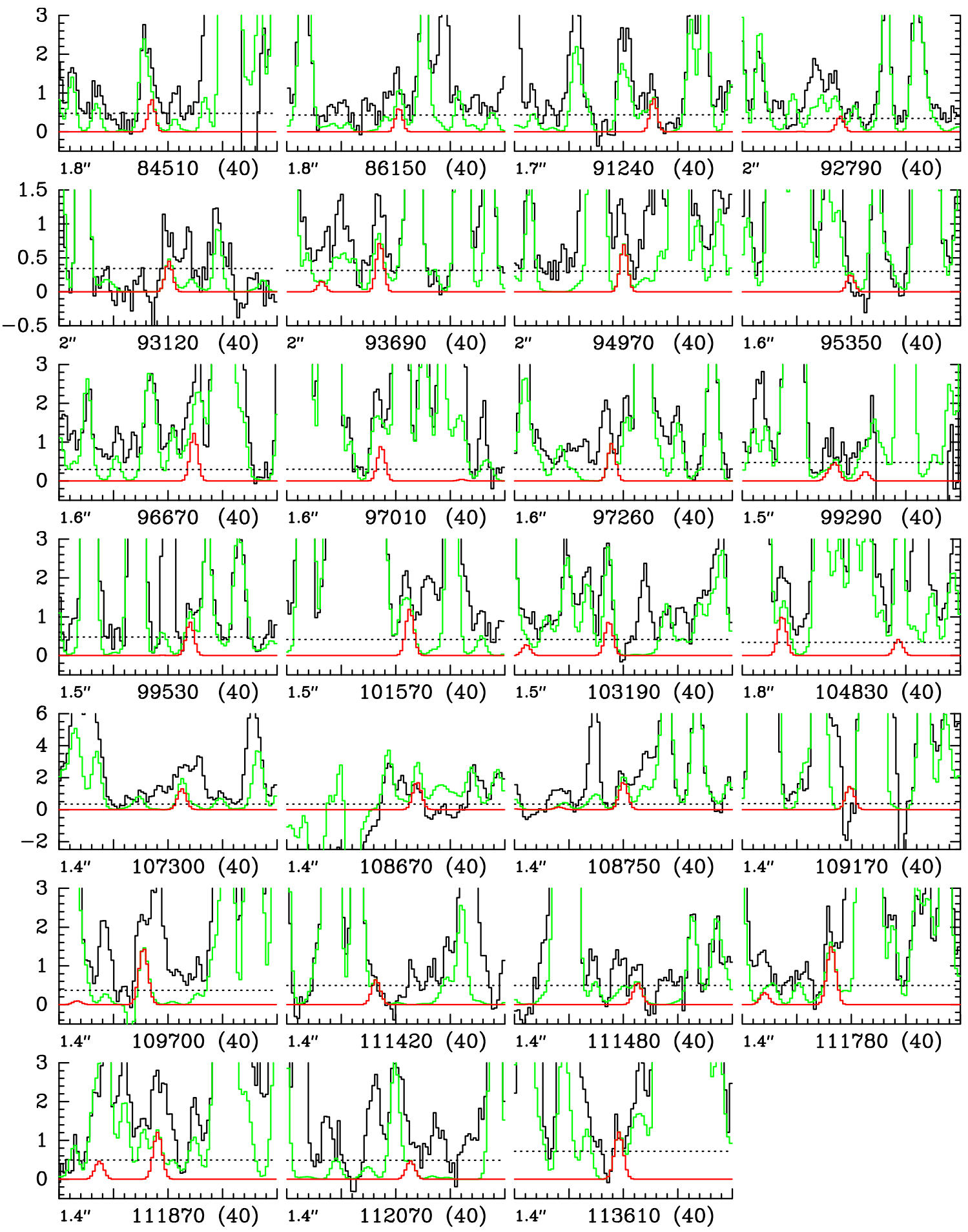

Fig. A.2. Same as Fig. A.1 for $\mathrm{CH}_{3} \mathrm{NHCHO}, v_{\mathrm{t}}=1$. 

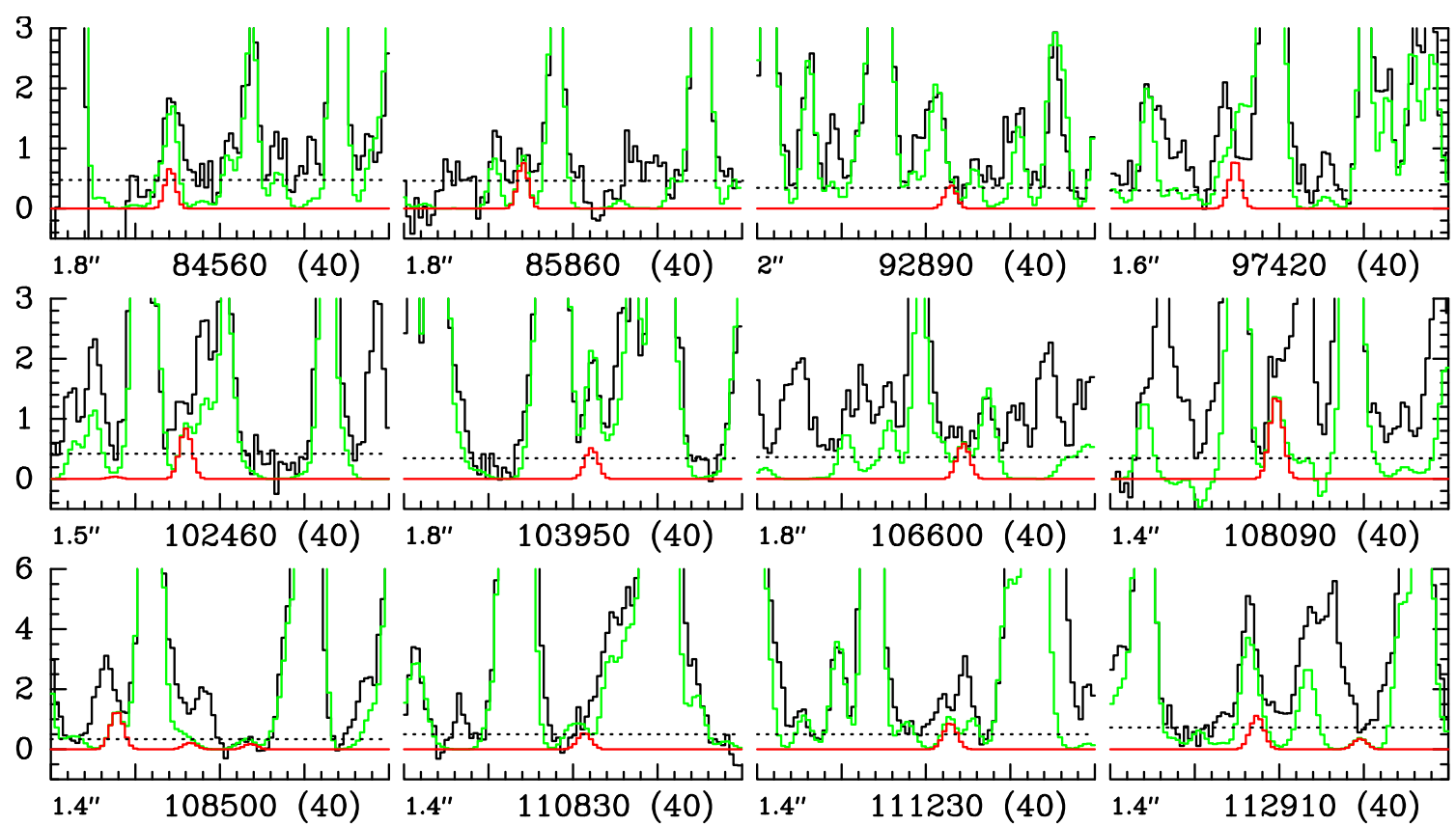

Fig. A.3. Same as Fig. A.1 for $\mathrm{CH}_{3} \mathrm{NHCHO}, v_{\mathrm{t}}=2$. 
A. Belloche et al.: Rotational spectroscopy, tentative interstellar detection, and chemical modeling of $\mathrm{N}$-methylformamide
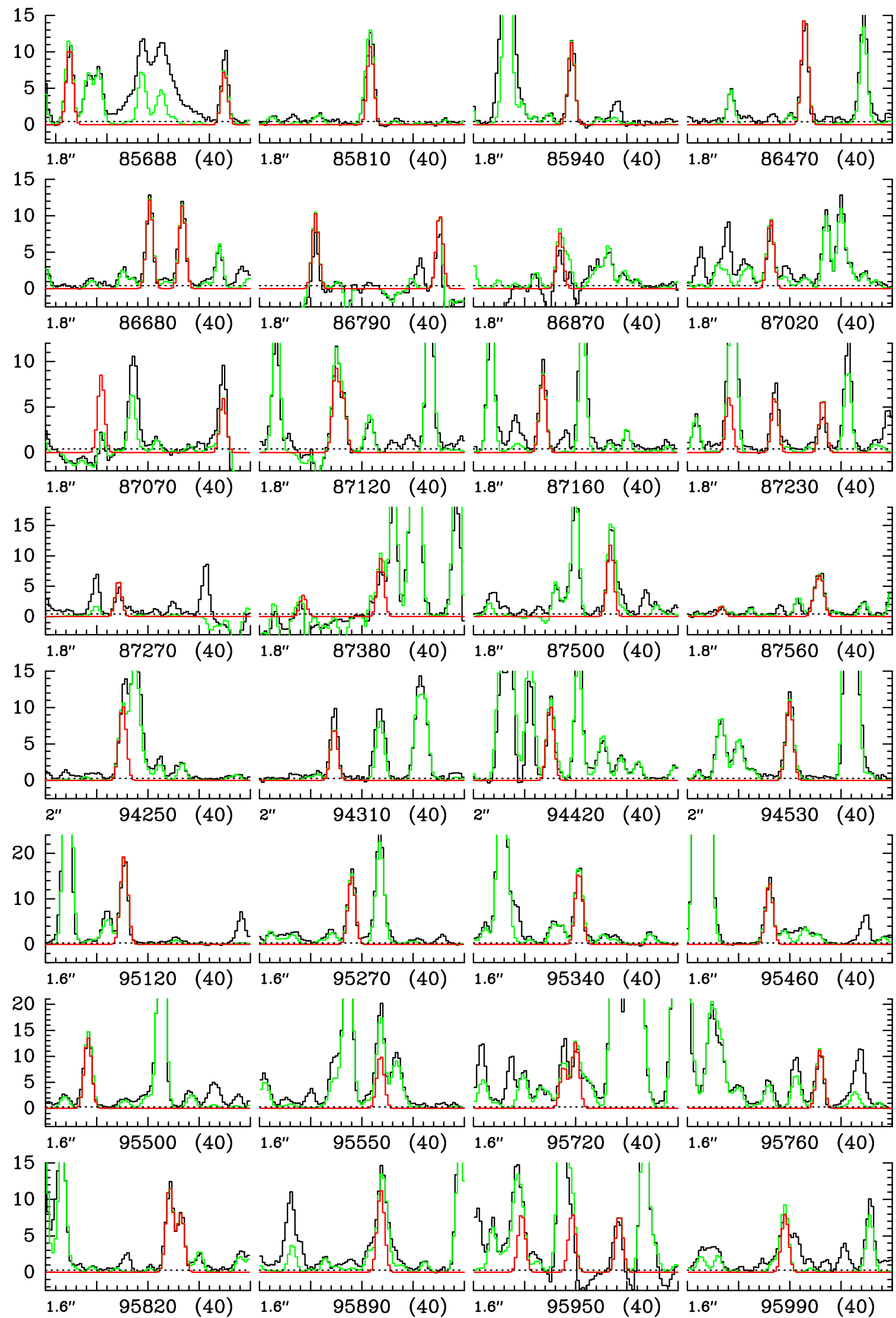

Fig. A.4. Same as Fig. A.1 for $\mathrm{CH}_{3} \mathrm{NCO}, v_{\mathrm{b}}=0$. 

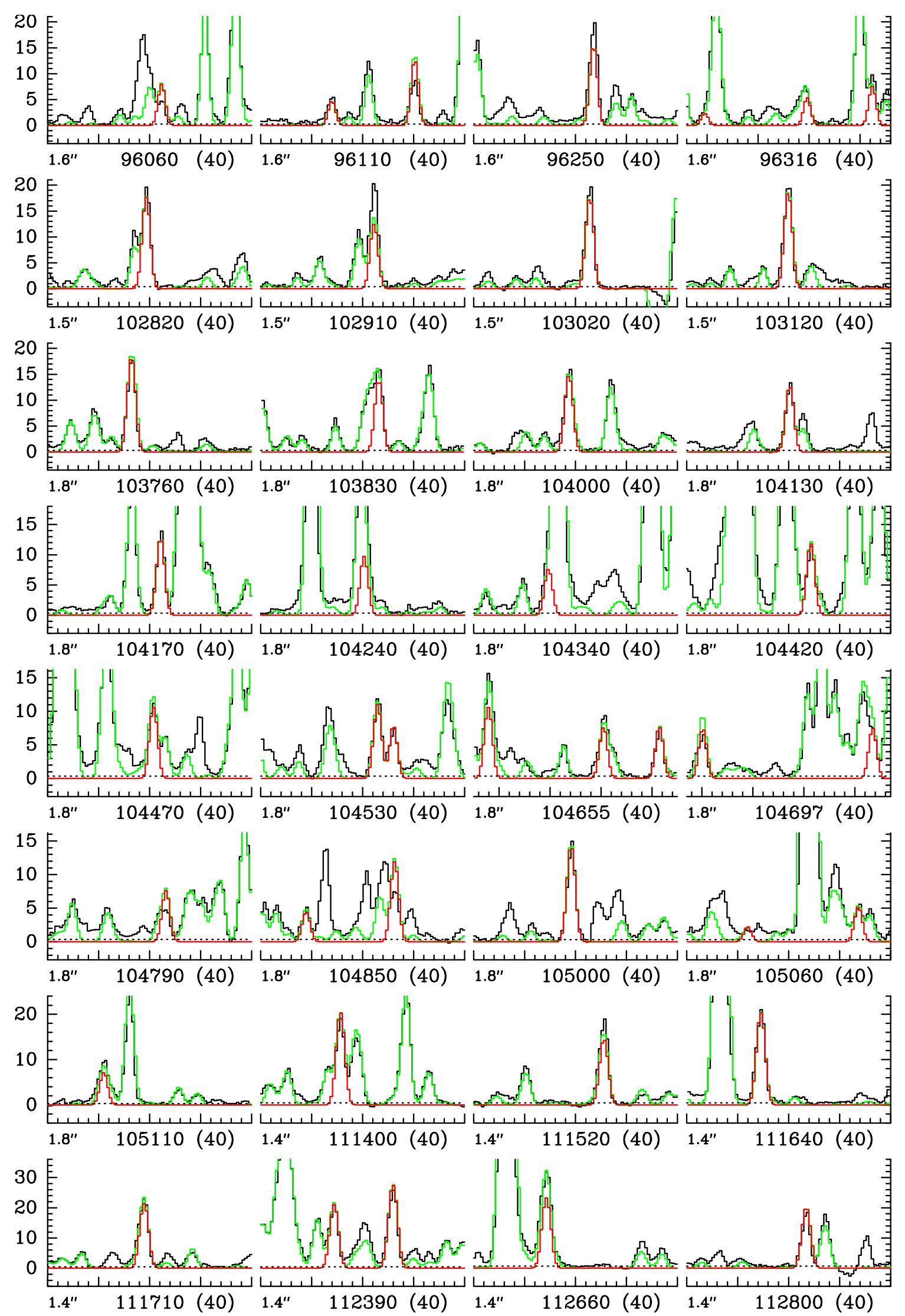

Fig. A.4. continued. 
A. Belloche et al.: Rotational spectroscopy, tentative interstellar detection, and chemical modeling of N-methylformamide
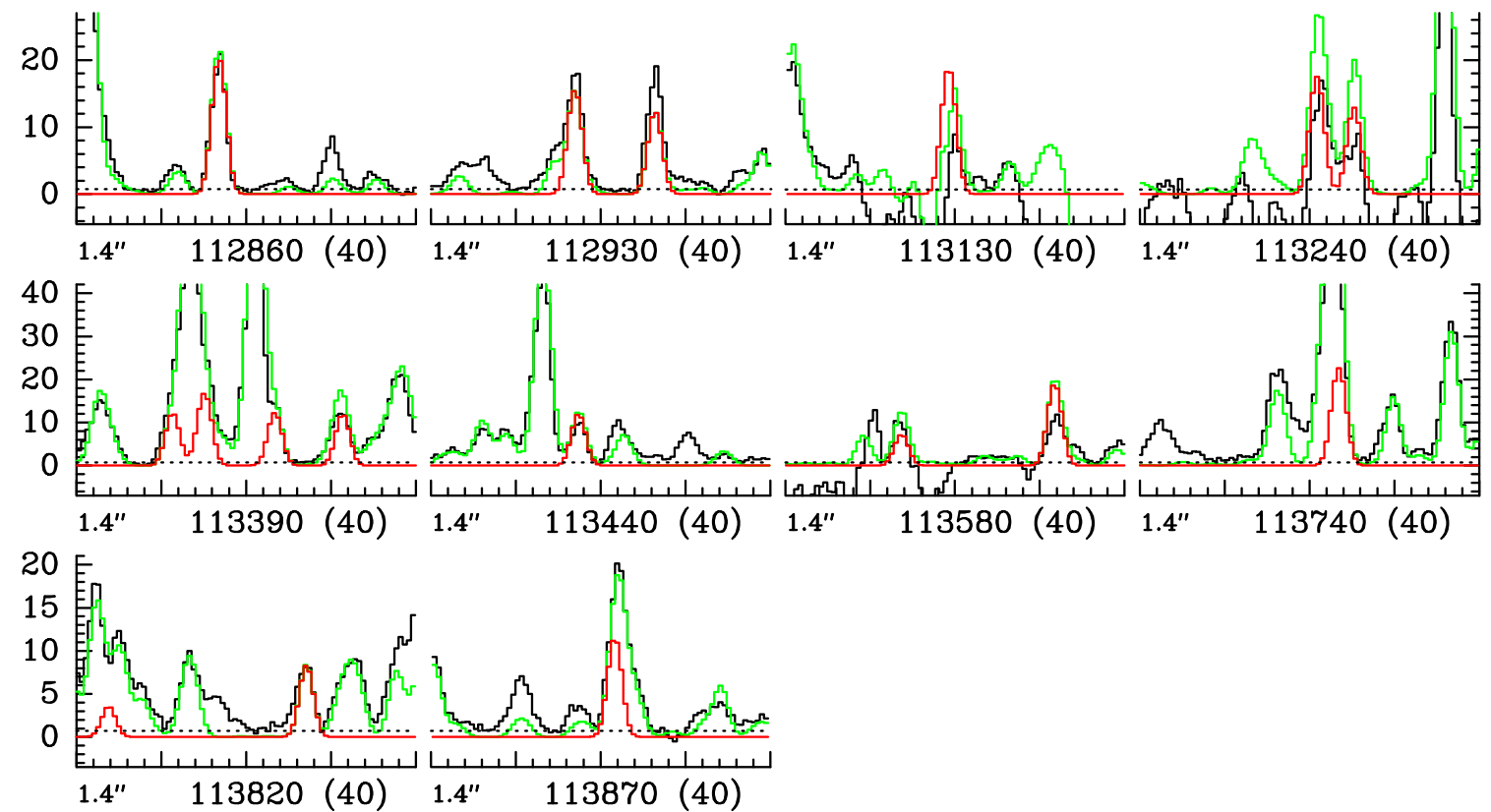

Fig. A.4. continued.
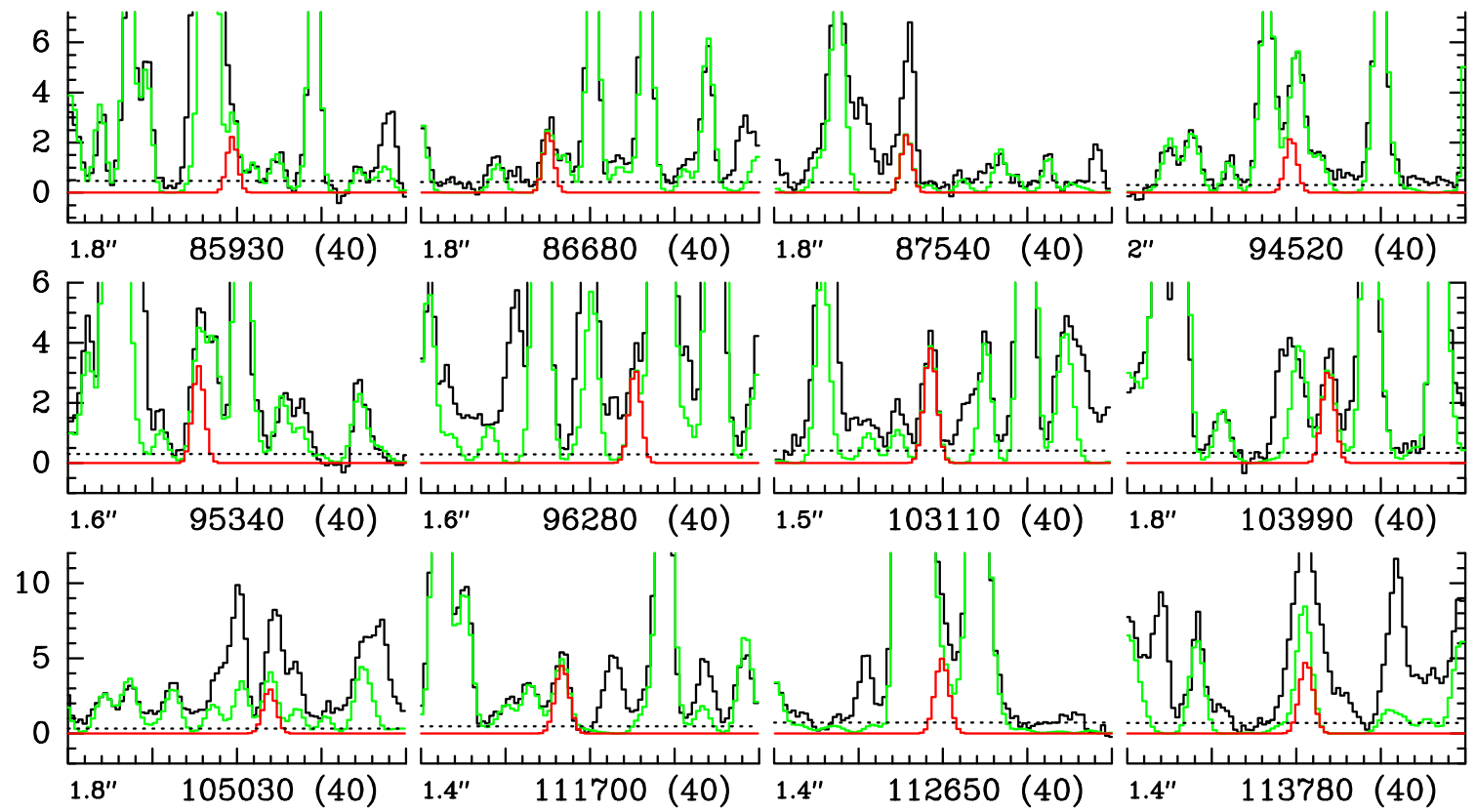

Fig. A.5. Same as Fig. A.1 for $\mathrm{CH}_{3} \mathrm{NCO}, v_{\mathrm{b}}=1$. 

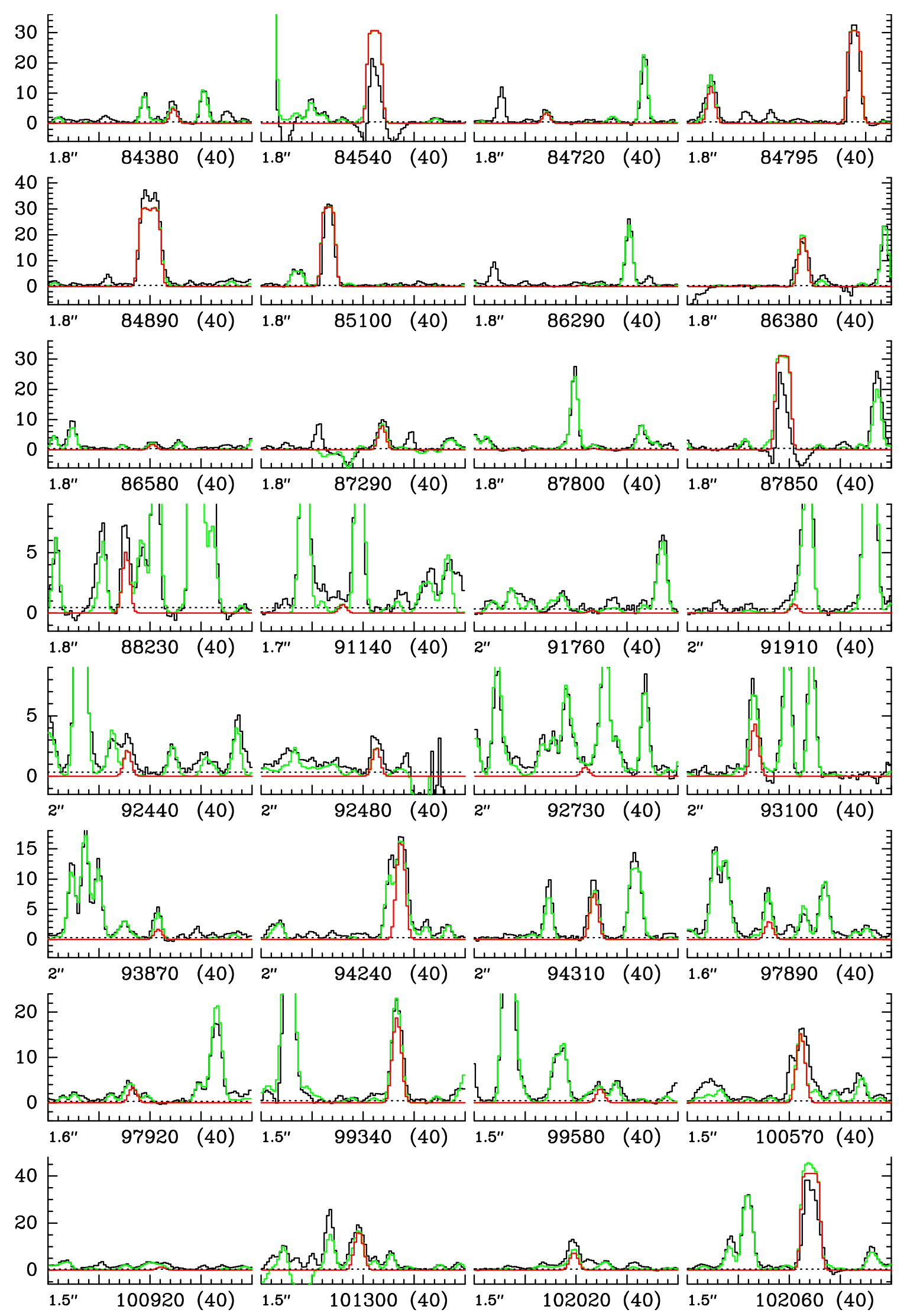

Fig. A.6. Same as Fig. A.1 for $\mathrm{NH}_{2} \mathrm{CHO}, v=0$. 
A. Belloche et al.: Rotational spectroscopy, tentative interstellar detection, and chemical modeling of N-methylformamide
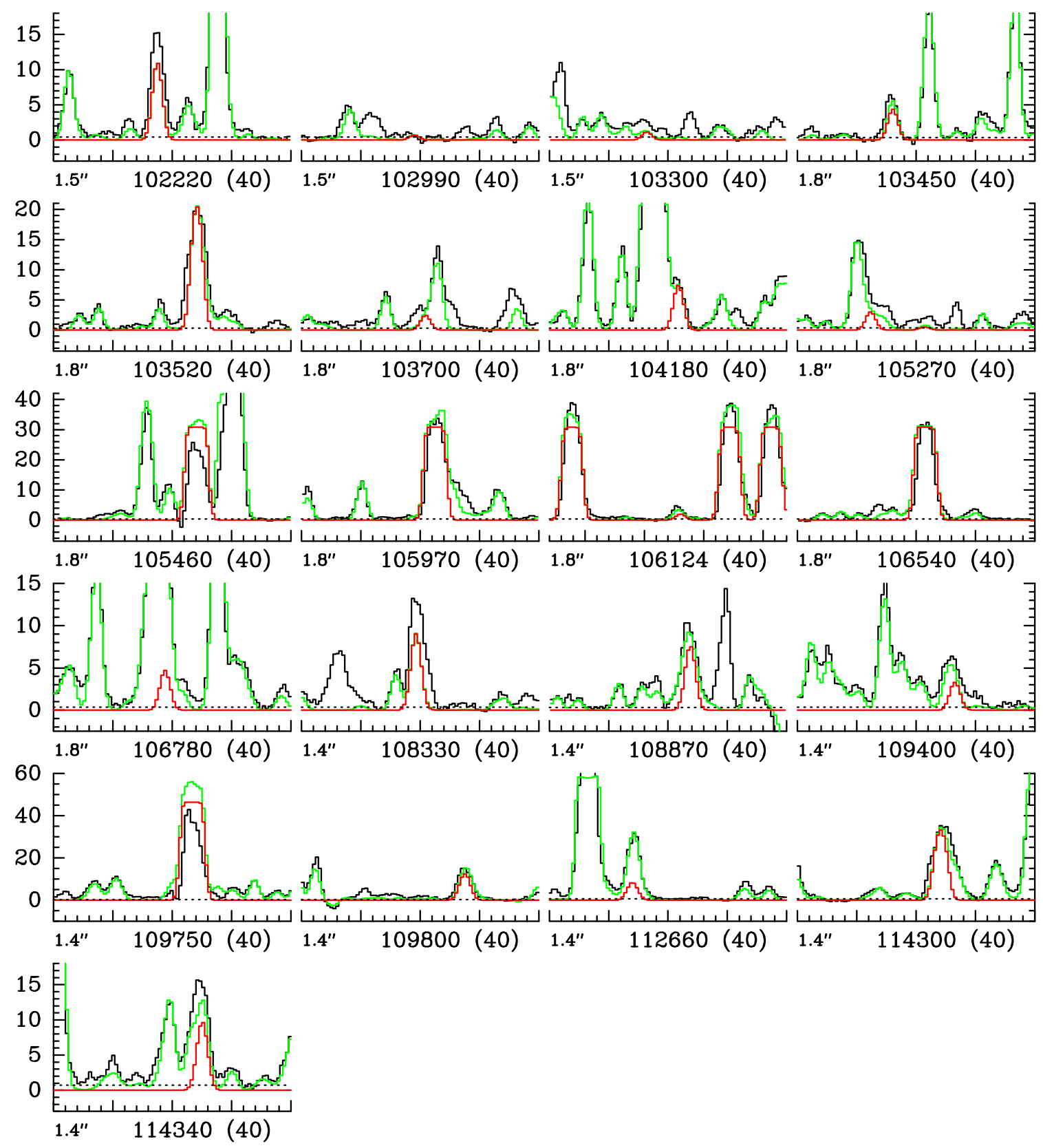

Fig. A.6. continued. 

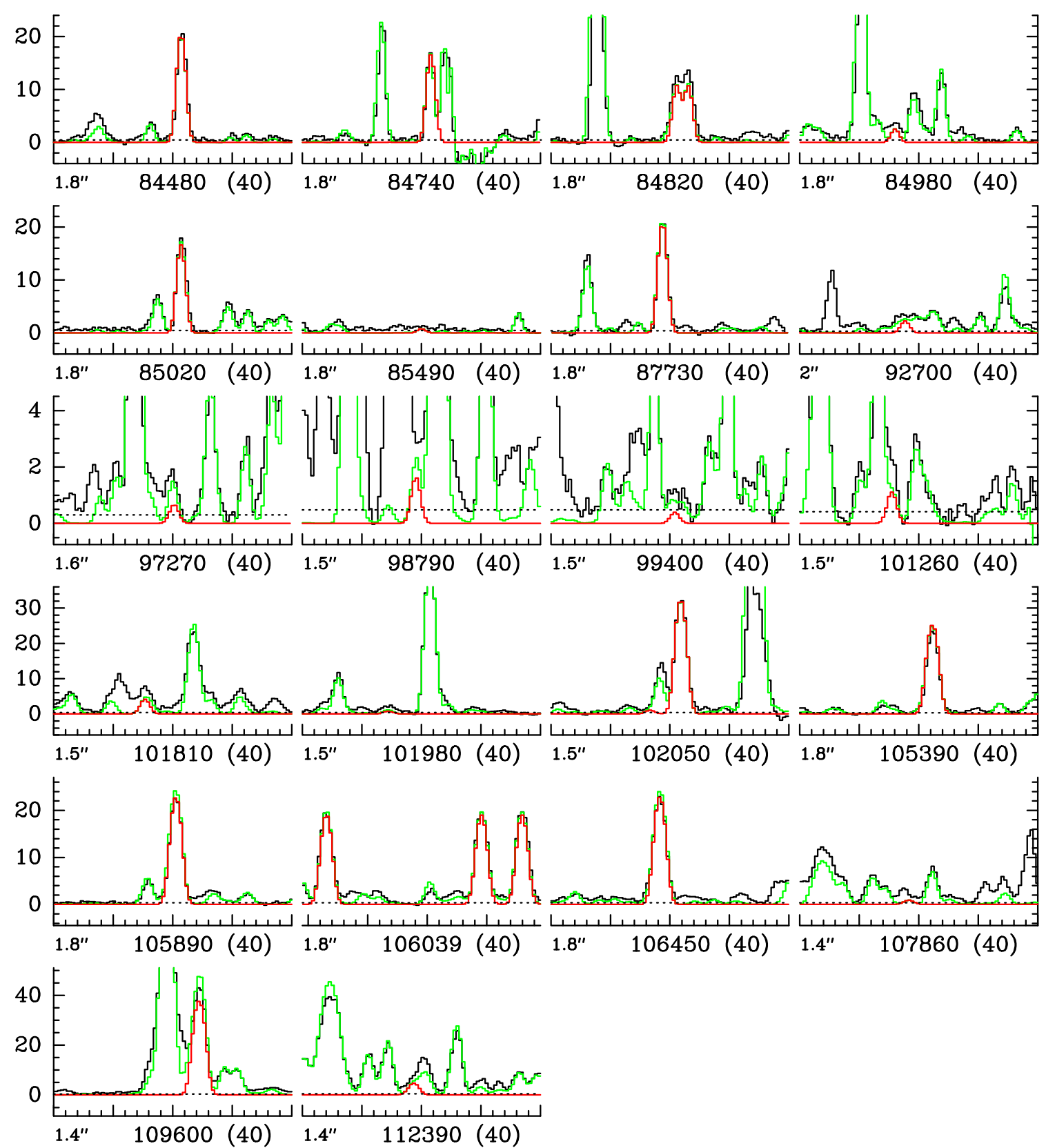

Fig. A.7. Same as Fig. A.1 for $\mathrm{NH}_{2} \mathrm{CHO}, v_{12}=1$. 
A. Belloche et al.: Rotational spectroscopy, tentative interstellar detection, and chemical modeling of N-methylformamide
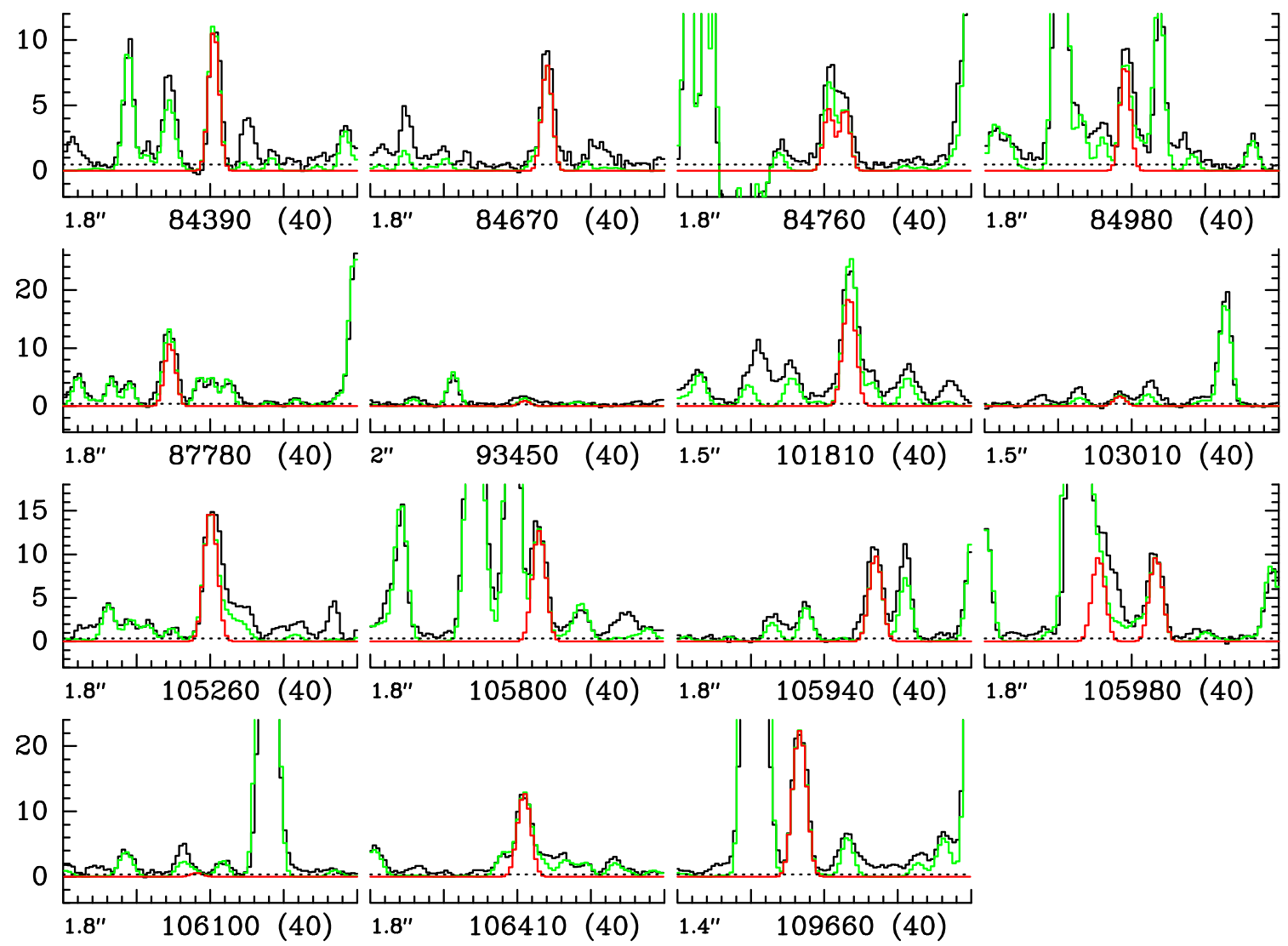

Fig. A.8. Same as Fig. A. 1 for $\mathrm{NH}_{2}{ }^{13} \mathrm{CHO}, v=0$.

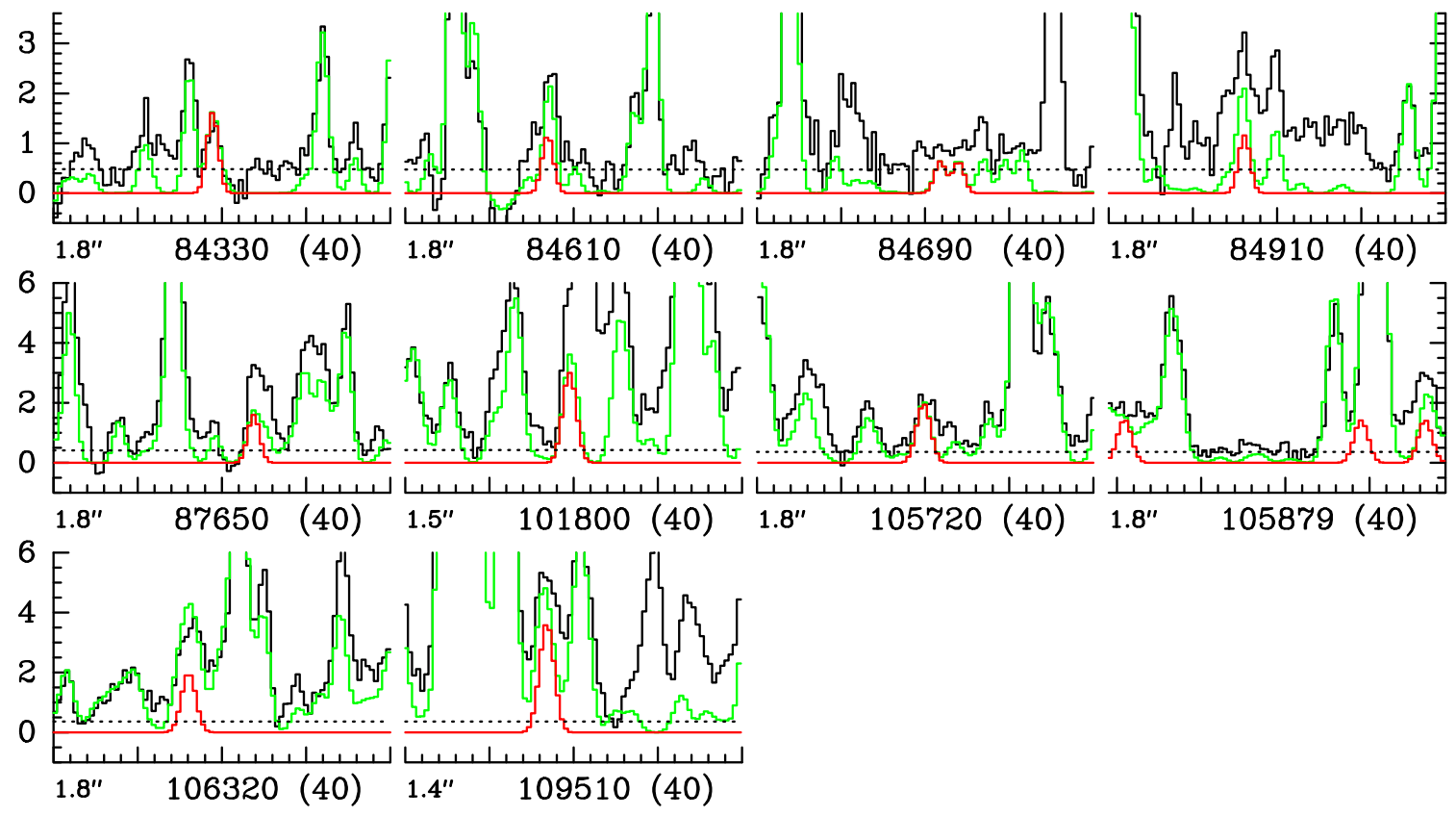

Fig. A.9. Same as Fig. A. 1 for $\mathrm{NH}_{2}{ }^{13} \mathrm{CHO}, v_{12}=1$. 


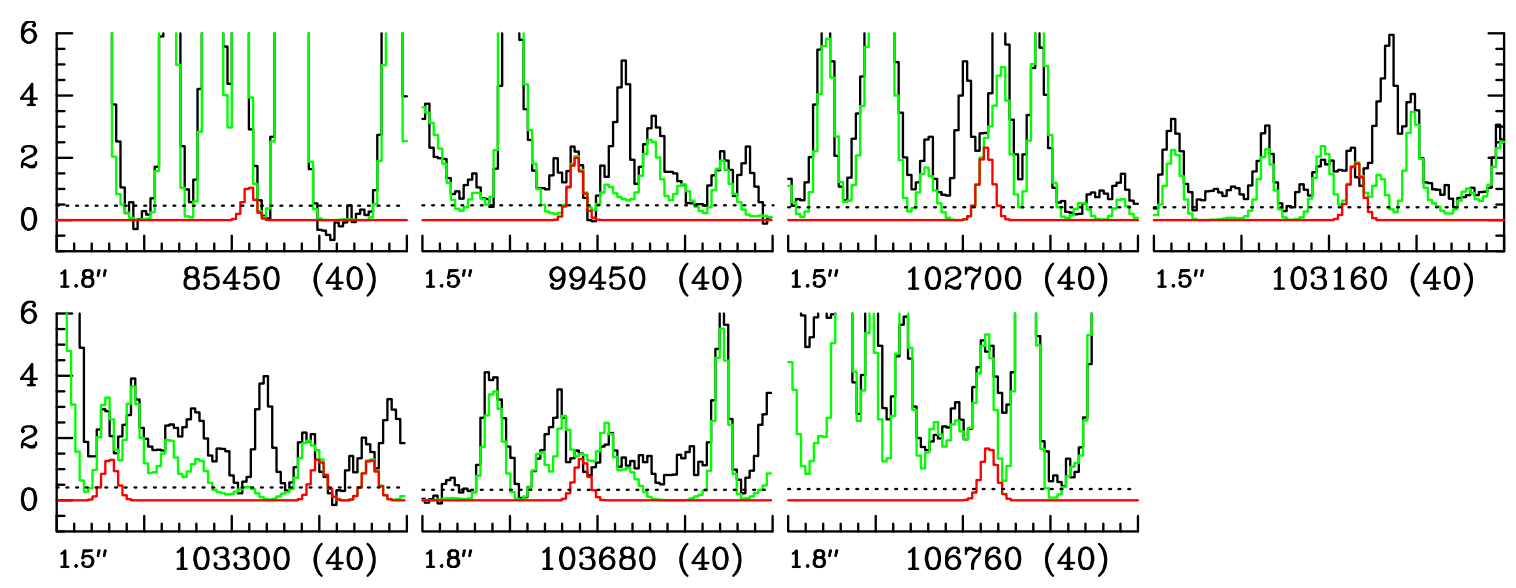

Fig. A.10. Same as Fig. A.1 for ${ }^{15} \mathrm{NH}_{2} \mathrm{CHO}, v=0$.
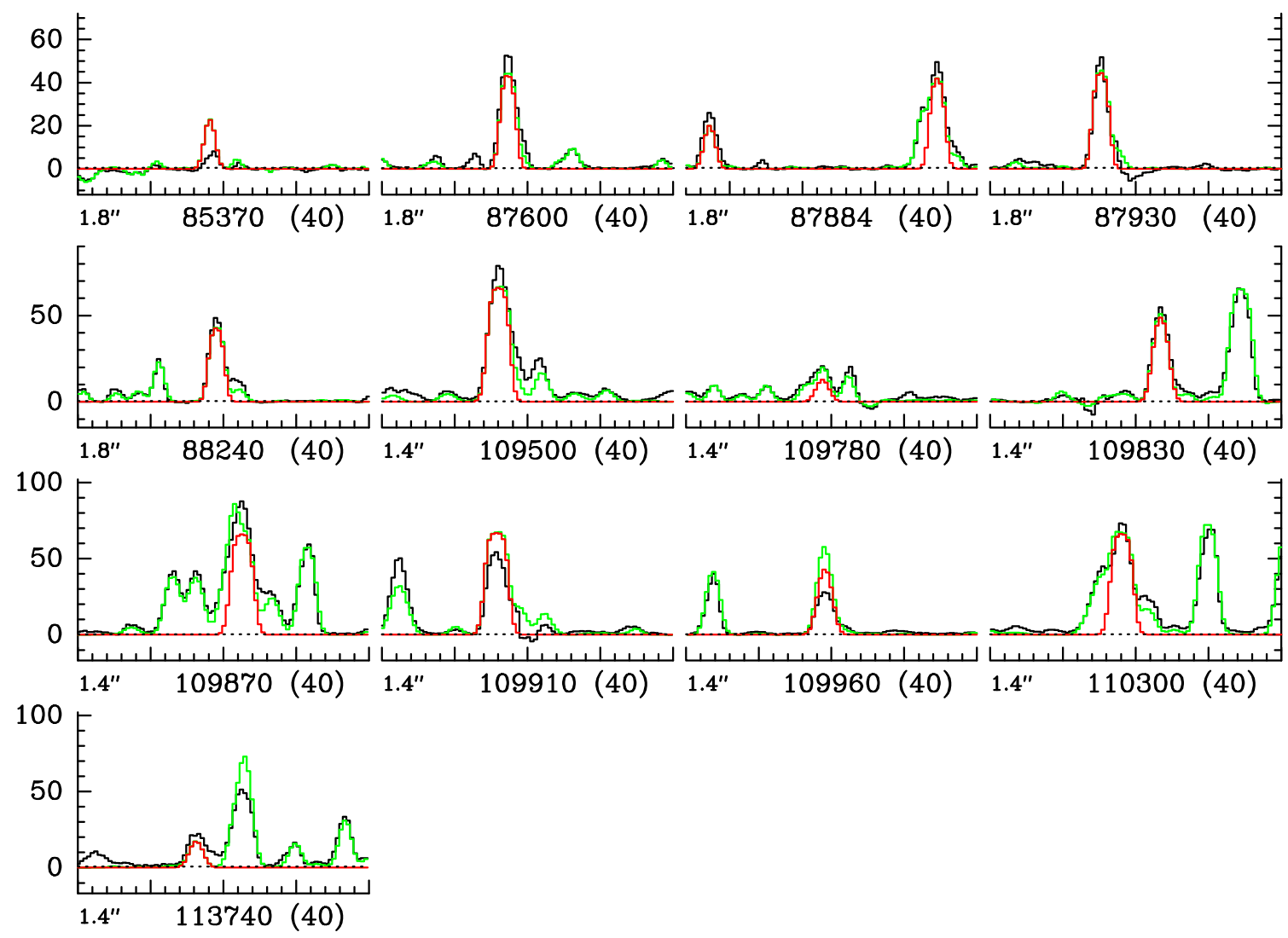

Fig. A.11. Same as Fig. A.1 for HNCO, $v=0$. 
A. Belloche et al.: Rotational spectroscopy, tentative interstellar detection, and chemical modeling of N-methylformamide
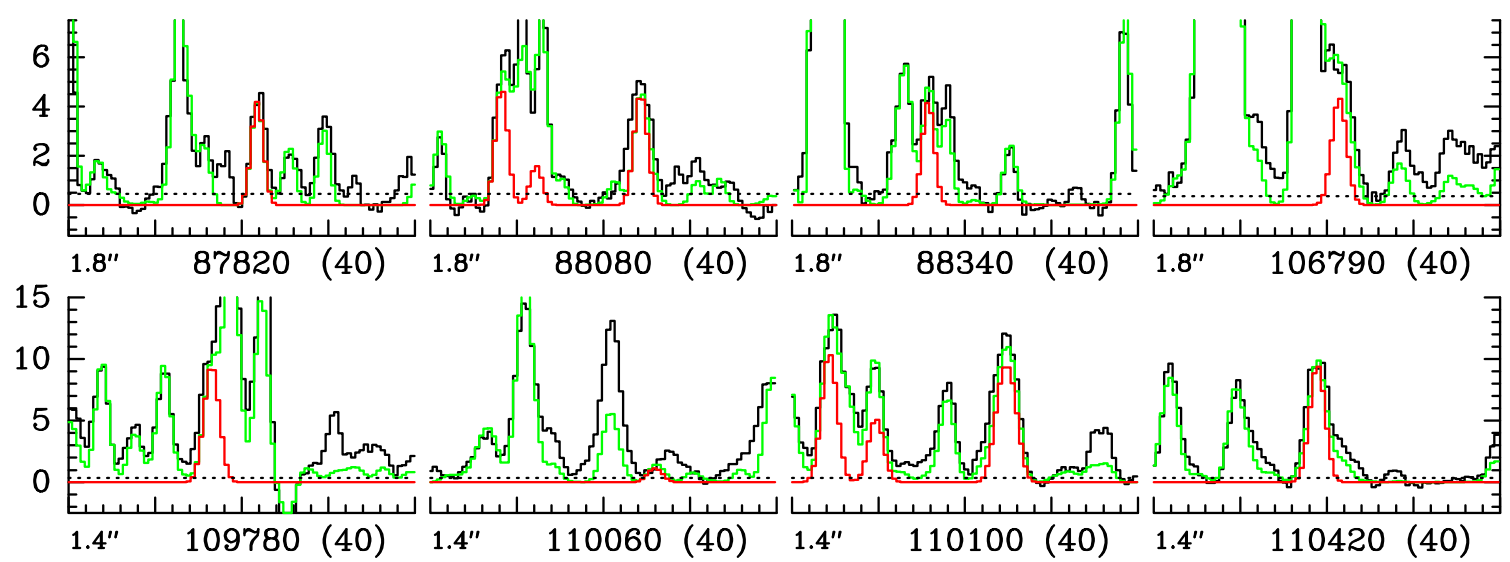

Fig. A.12. Same as Fig. A.1 for $\mathrm{HNCO}, v_{5}=1$.

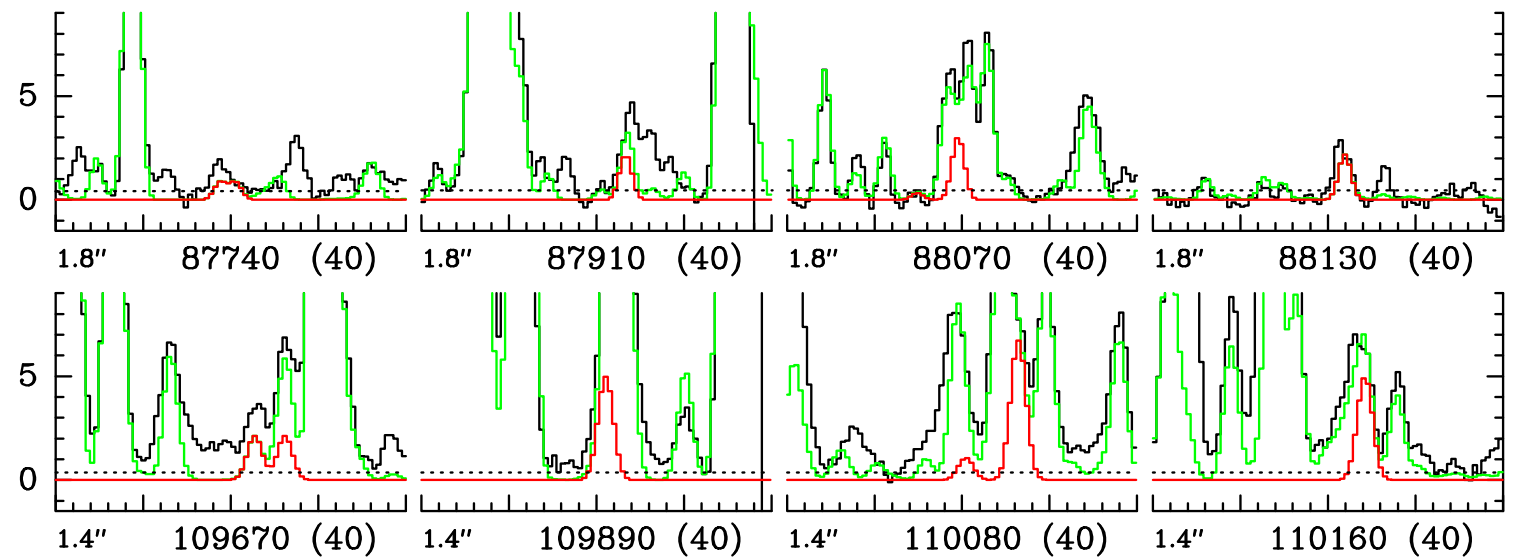

Fig. A.13. Same as Fig. A.1 for $\mathrm{HNCO}, v_{6}=1$.

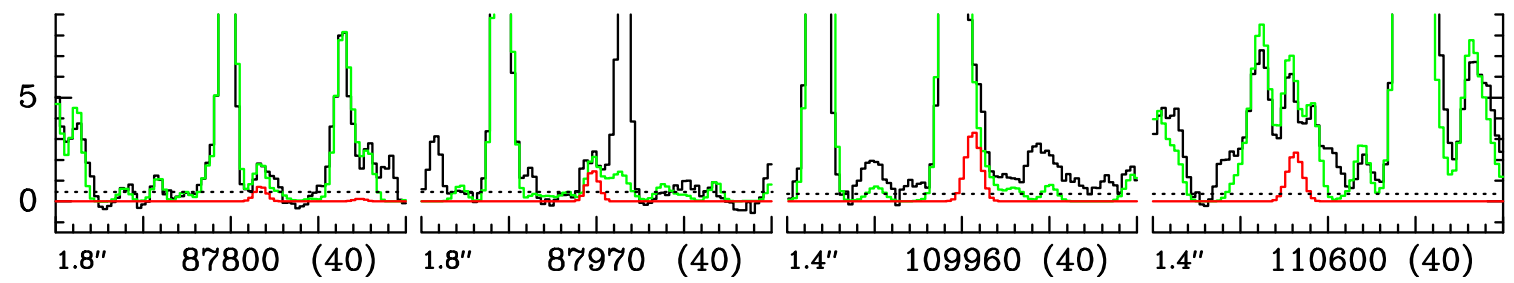

Fig. A.14. Same as Fig. A.1 for $\mathrm{HNCO}, v_{4}=1$. 
A\&A 601, A49 (2017)
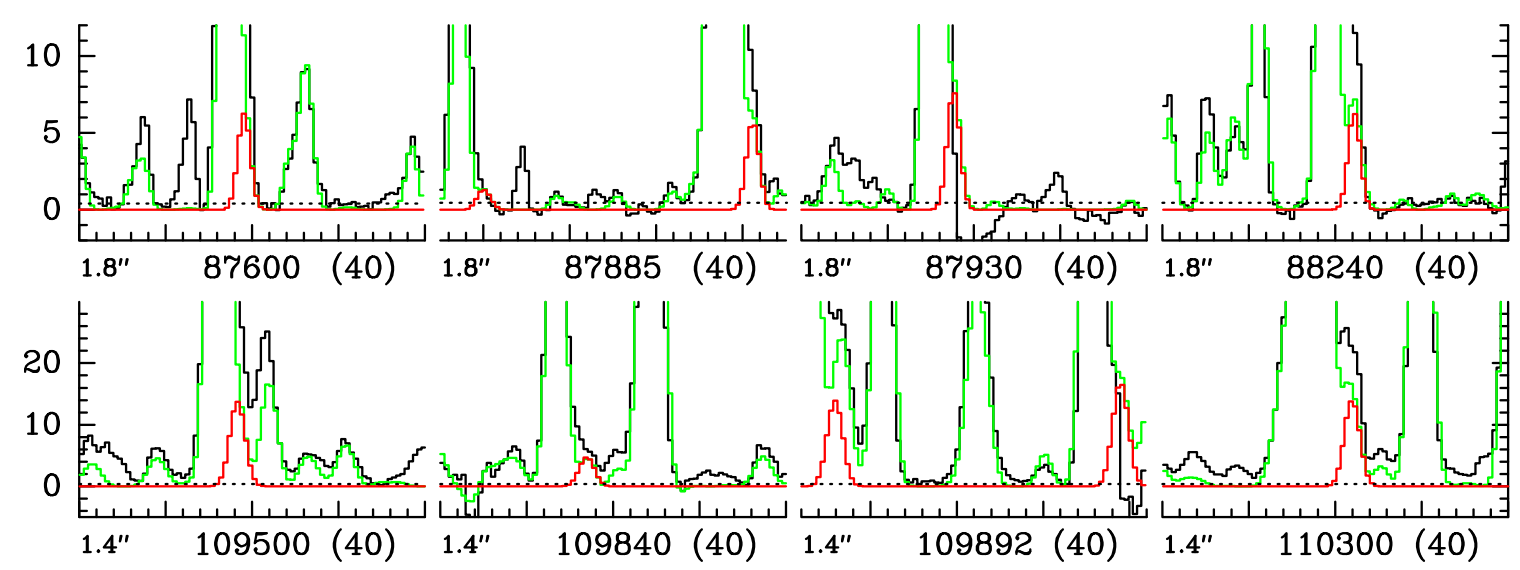

Fig. A.15. Same as Fig. A.1 for $\mathrm{HN}^{13} \mathrm{CO}, v=0$. 
A. Belloche et al.: Rotational spectroscopy, tentative interstellar detection, and chemical modeling of $\mathrm{N}$-methylformamide
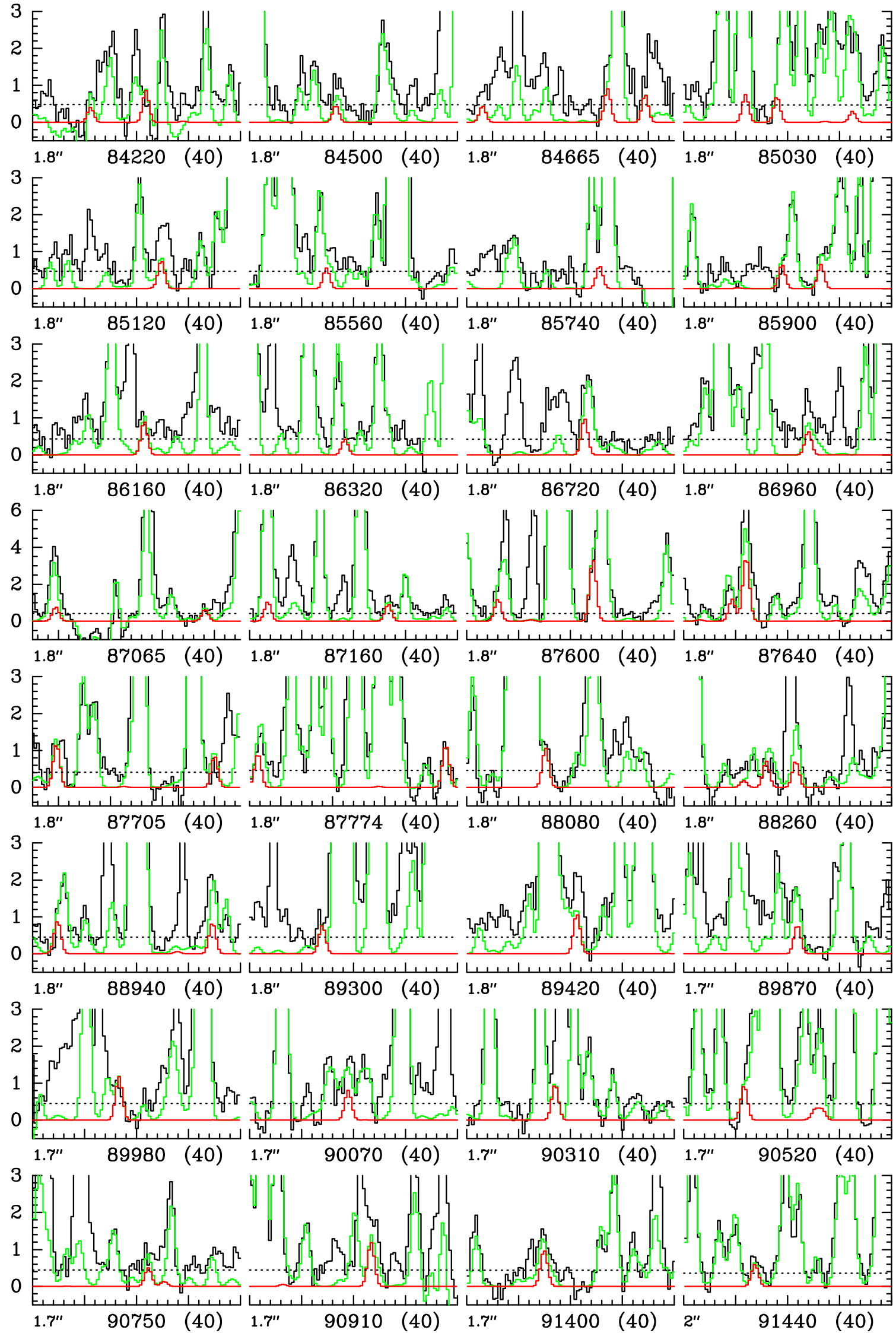

Fig. A.16. Same as Fig. A.1 for $\mathrm{CH}_{3} \mathrm{CONH}_{2}, v_{\mathrm{t}}=0$. 

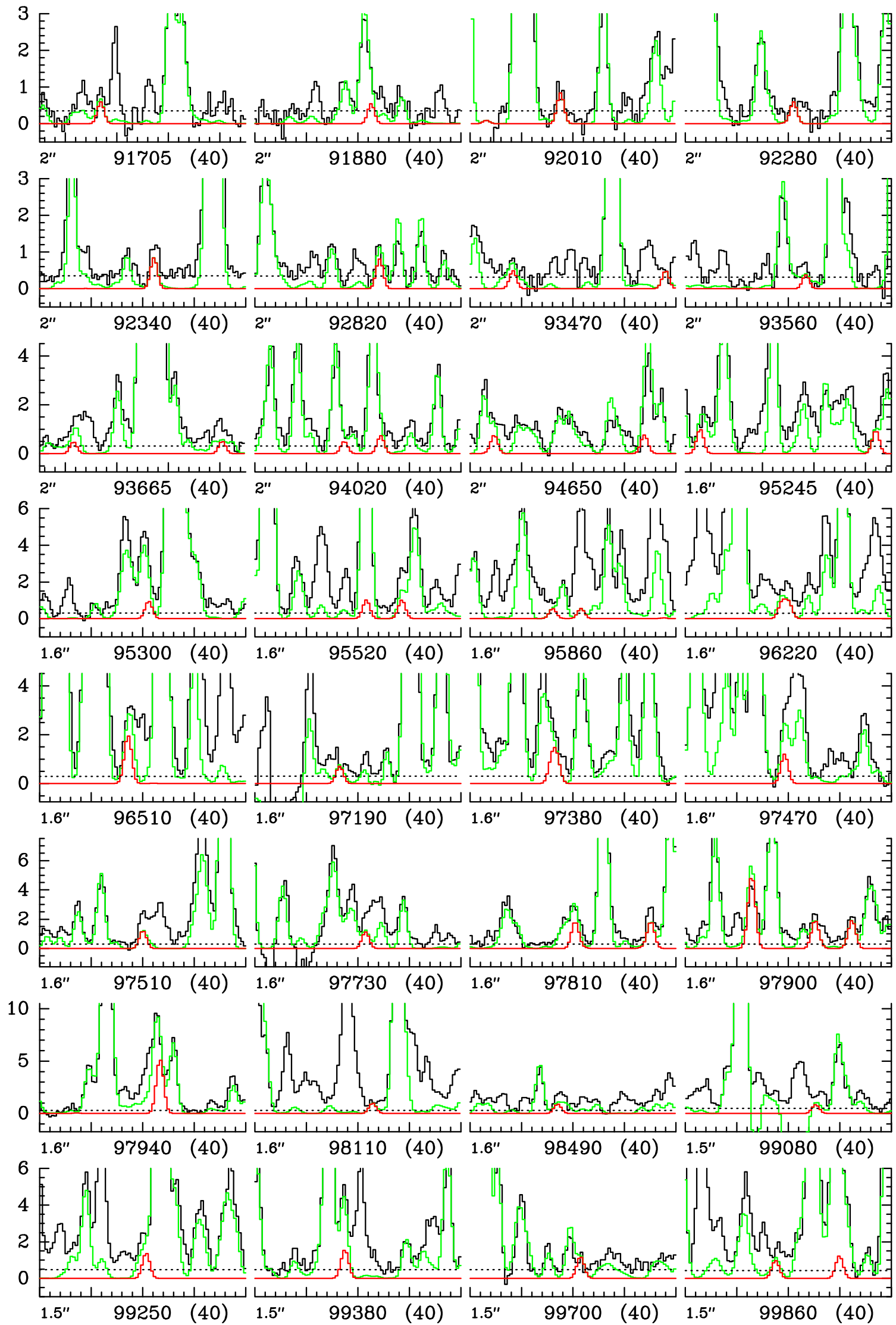

Fig. A.16. continued. 
A. Belloche et al.: Rotational spectroscopy, tentative interstellar detection, and chemical modeling of $\mathrm{N}$-methylformamide
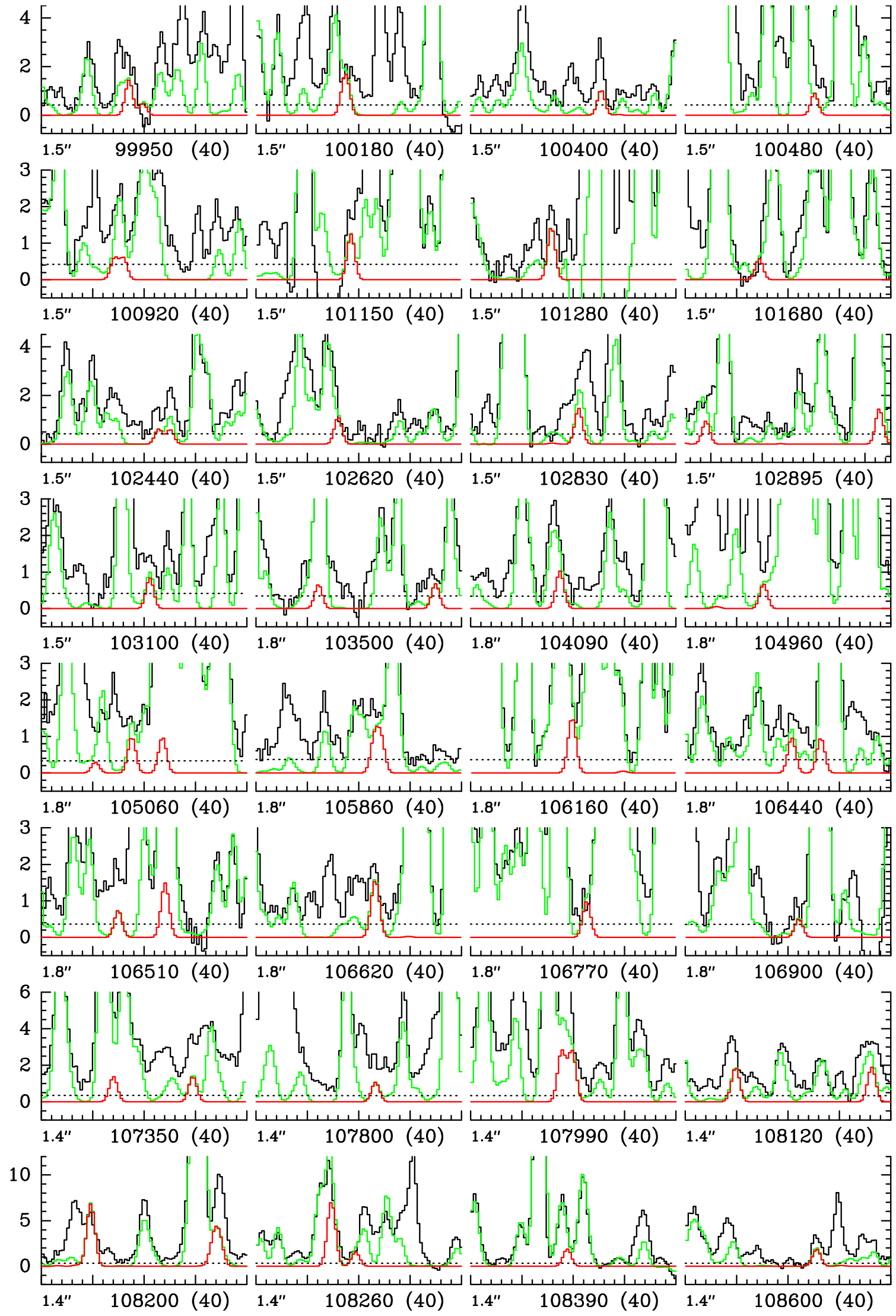

Fig. A.16. continued. 

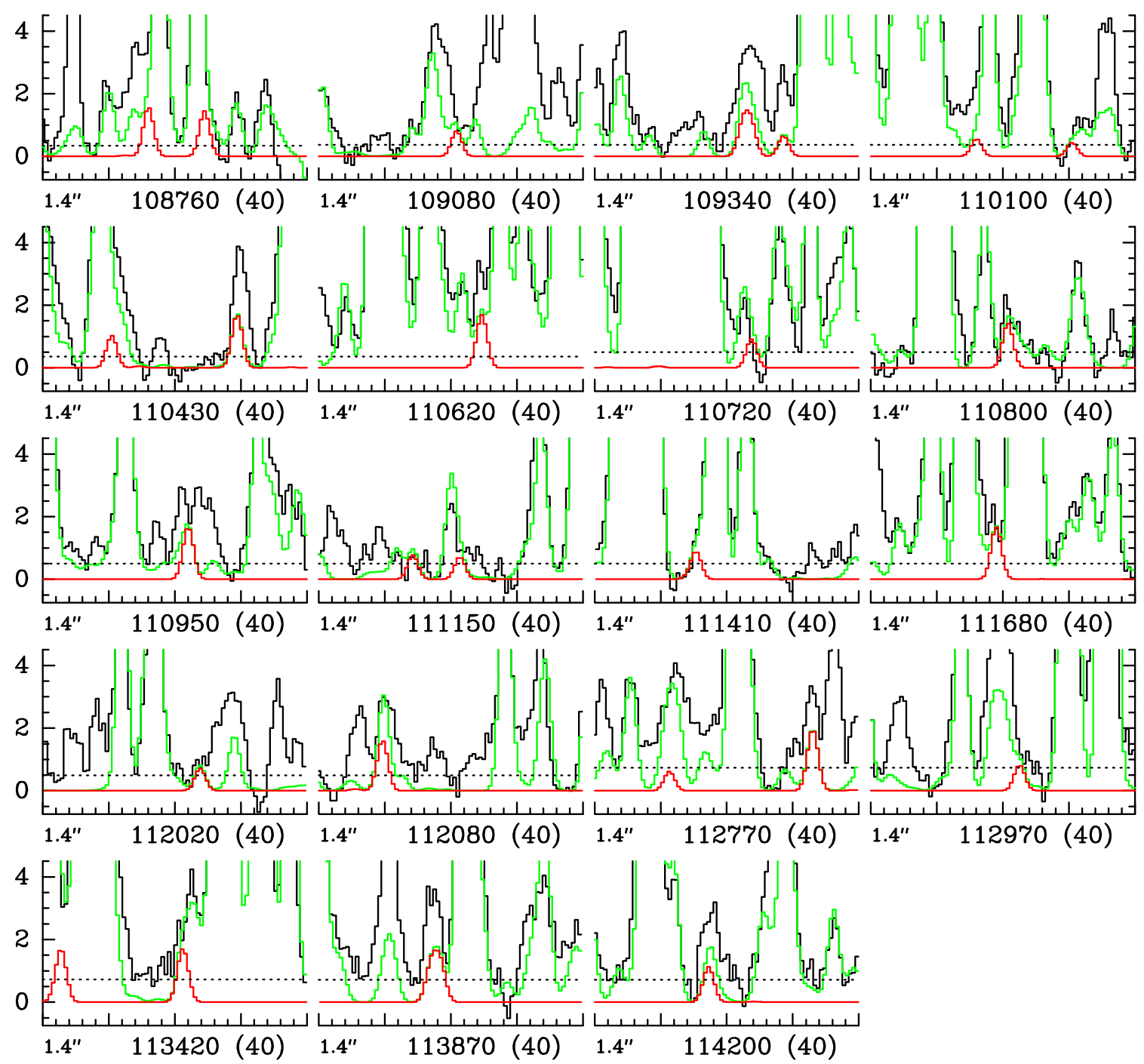

Fig. A.16. continued. 
A. Belloche et al.: Rotational spectroscopy, tentative interstellar detection, and chemical modeling of $\mathrm{N}$-methylformamide
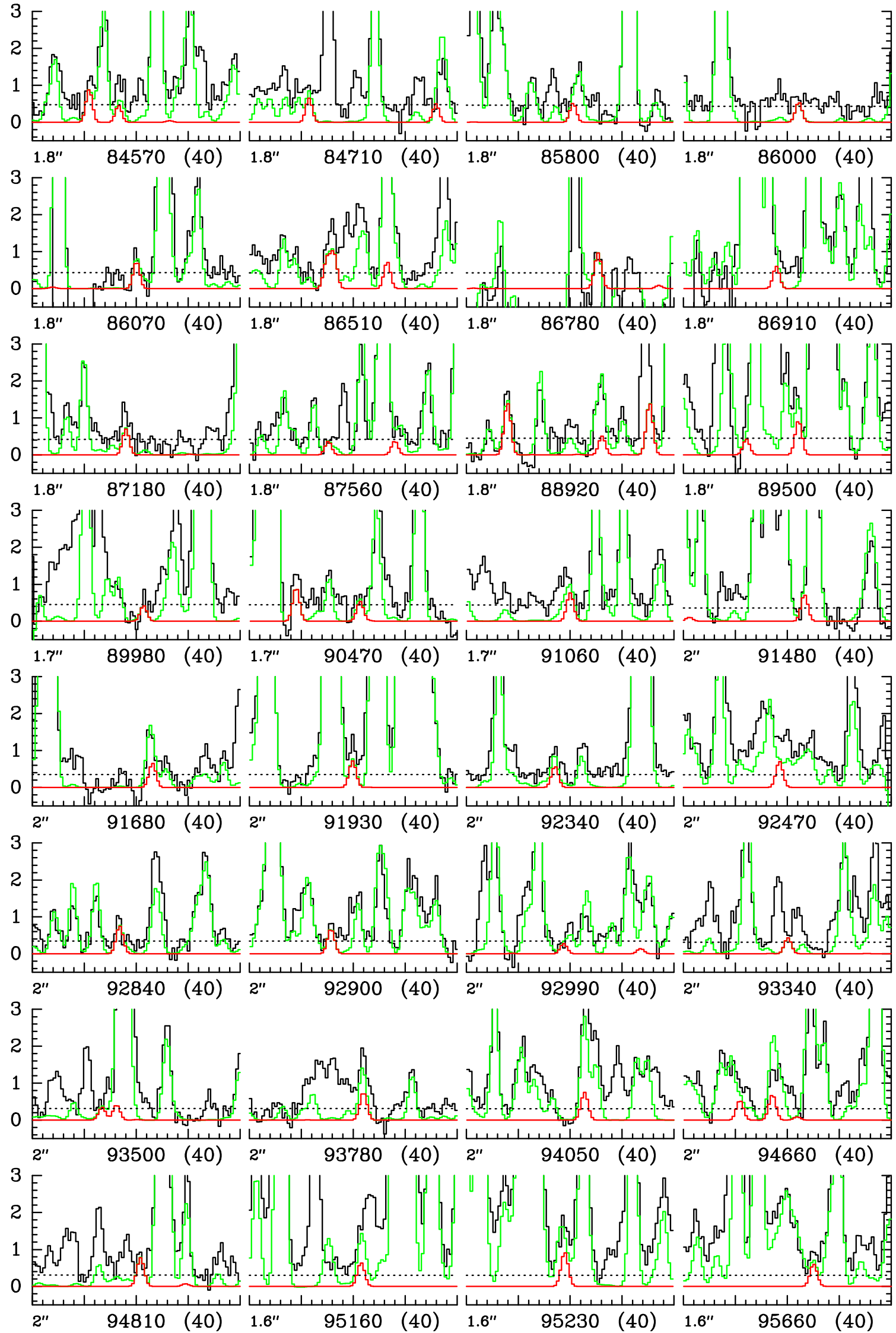

Fig. A.17. Same as Fig. A.1 for $\mathrm{CH}_{3} \mathrm{CONH}_{2}, v_{\mathrm{t}}=1$. 

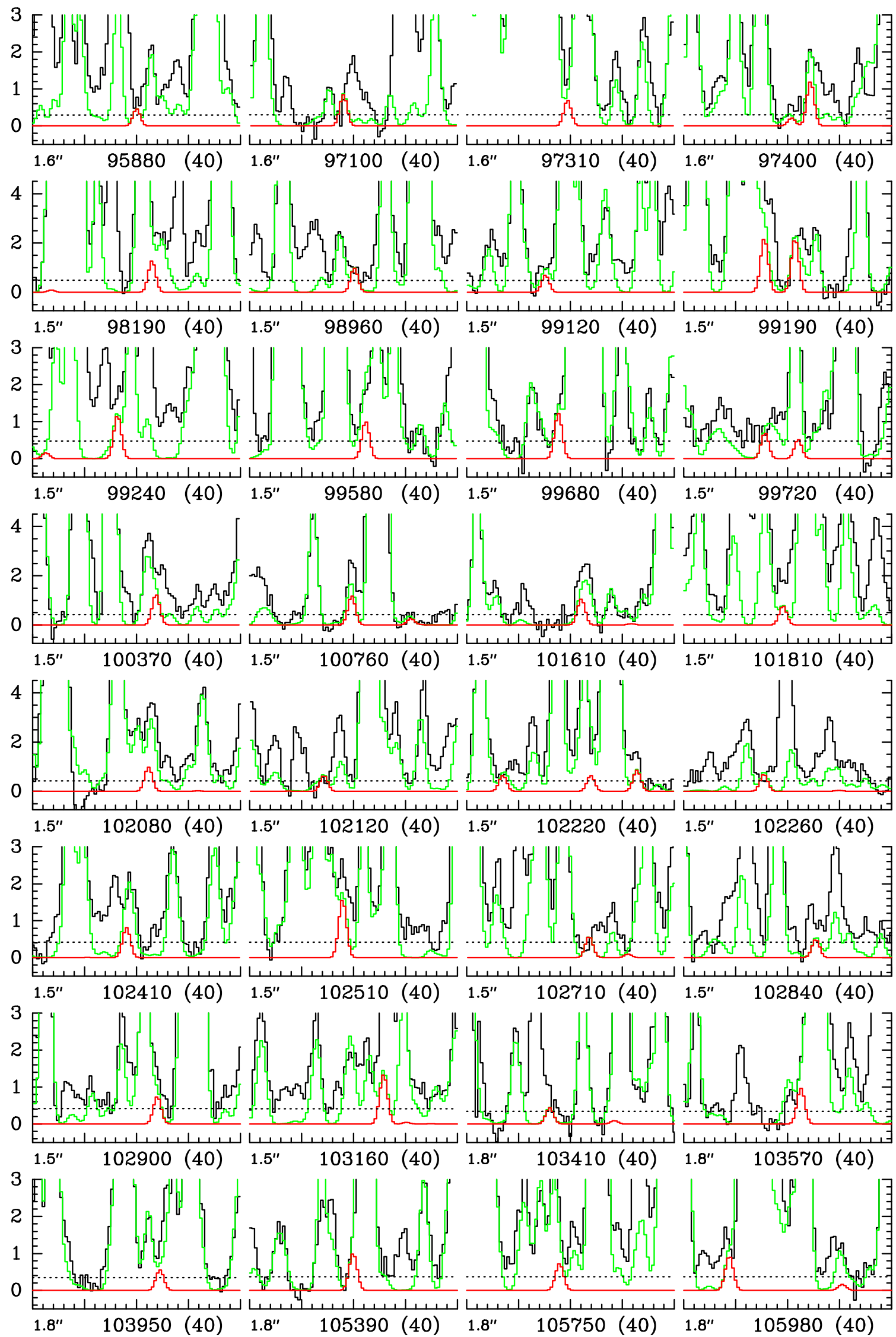

Fig. A.17. continued. 
A. Belloche et al.: Rotational spectroscopy, tentative interstellar detection, and chemical modeling of N-methylformamide
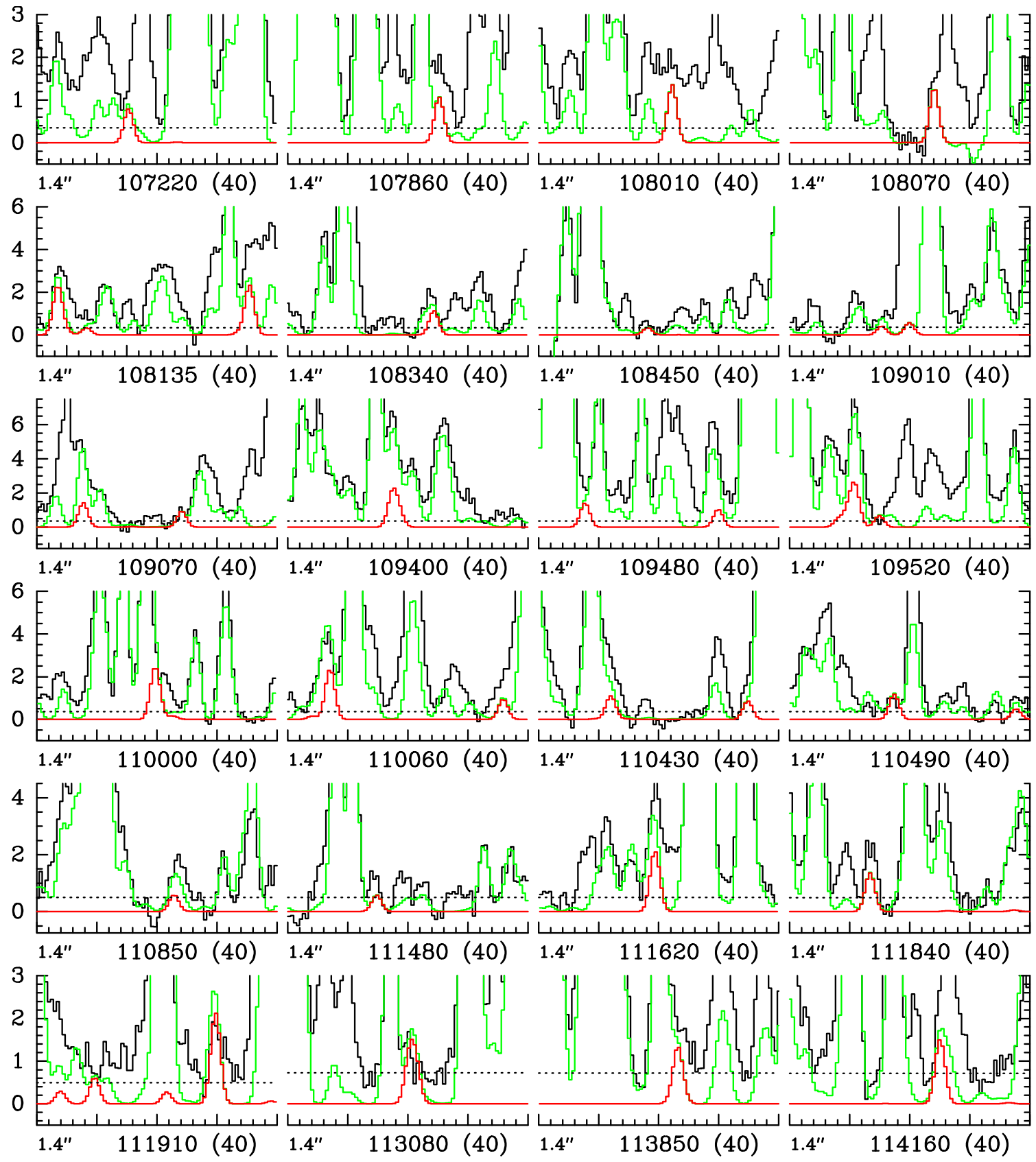

Fig. A.17. continued. 

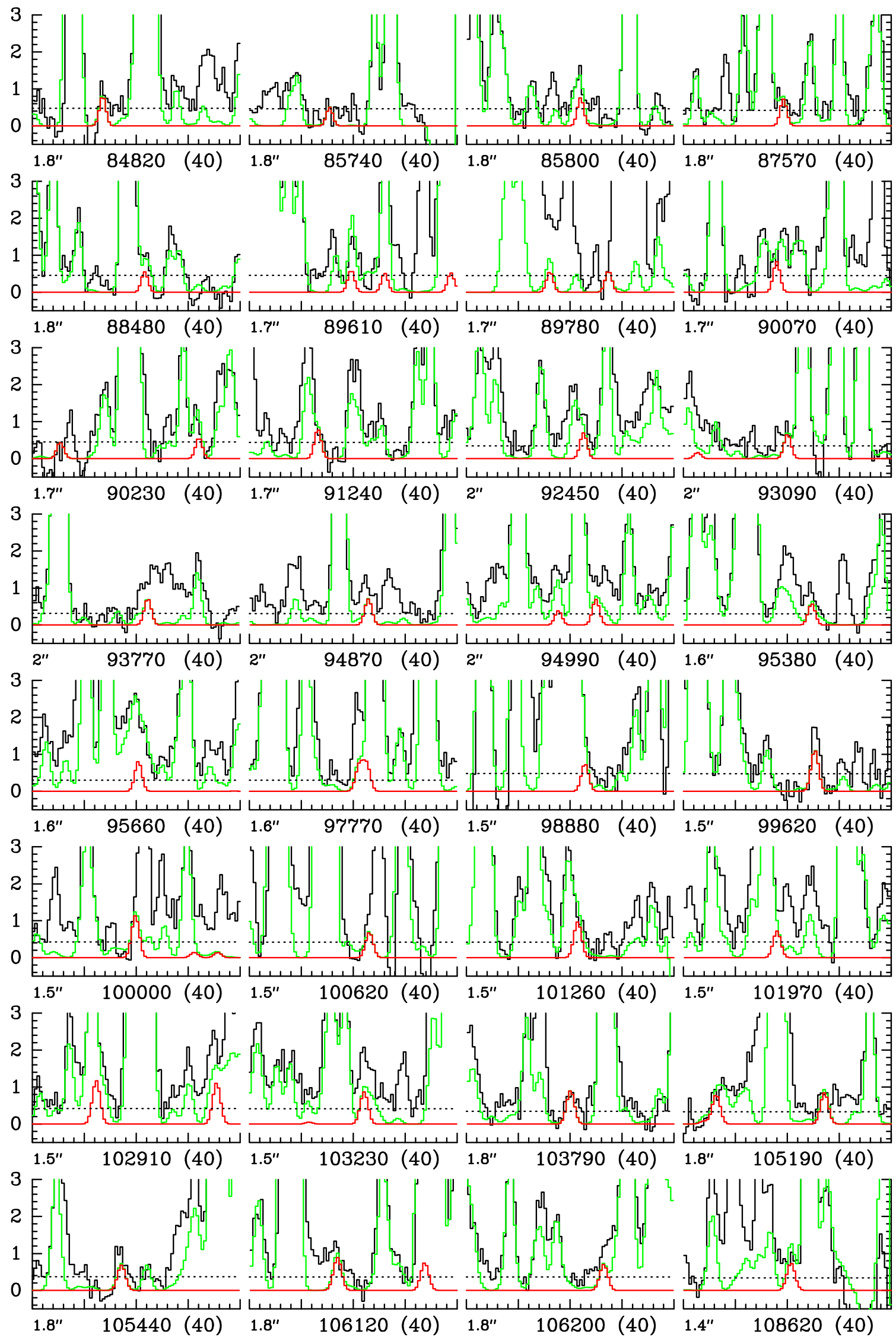

Fig. A.18. Same as Fig. A. 1 for $\mathrm{CH}_{3} \mathrm{CONH}_{2}, v_{\mathrm{t}}=2$. 
A. Belloche et al.: Rotational spectroscopy, tentative interstellar detection, and chemical modeling of N-methylformamide
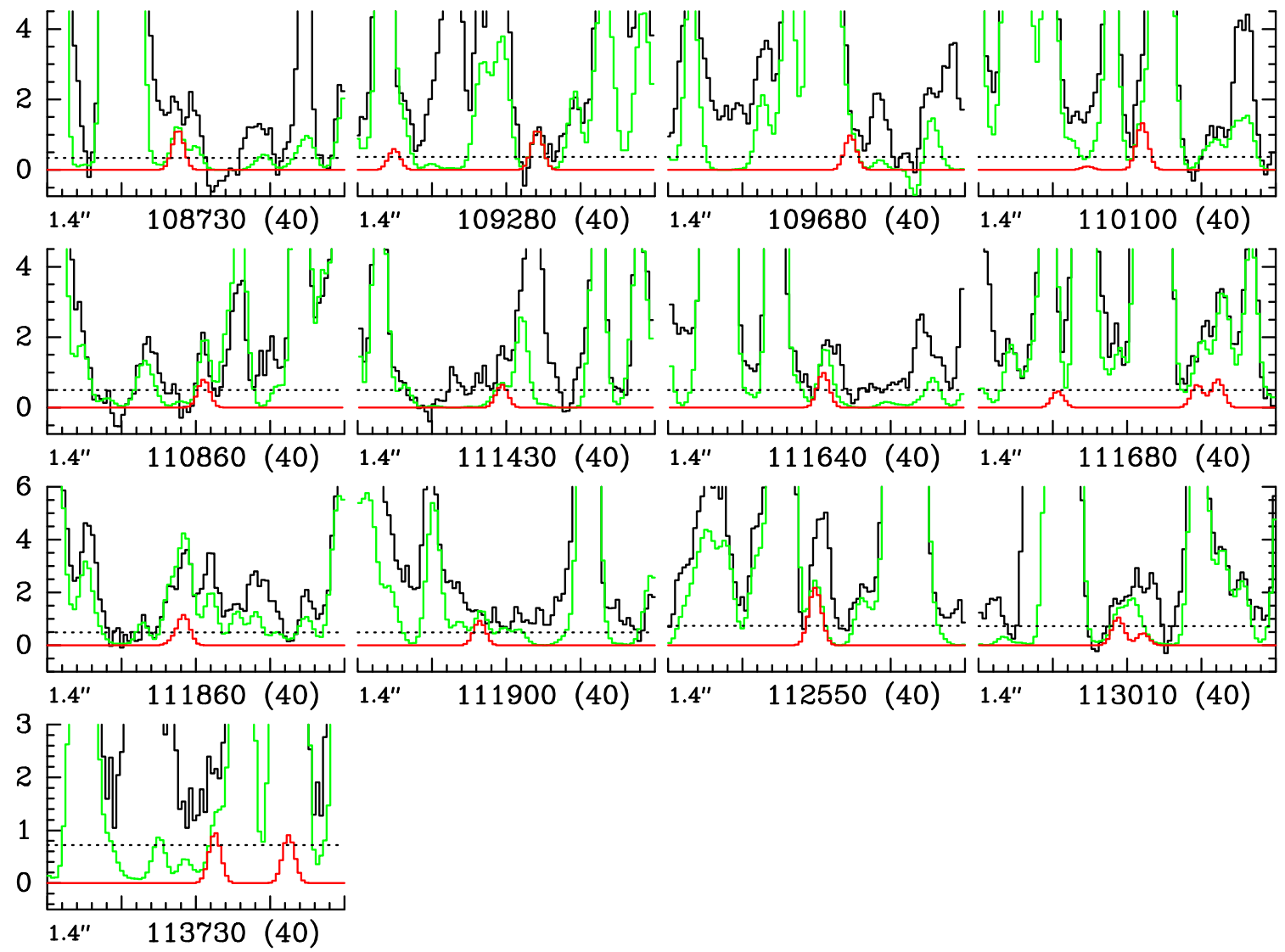

Fig. A.18. continued. 
A\&A 601, A49 (2017)

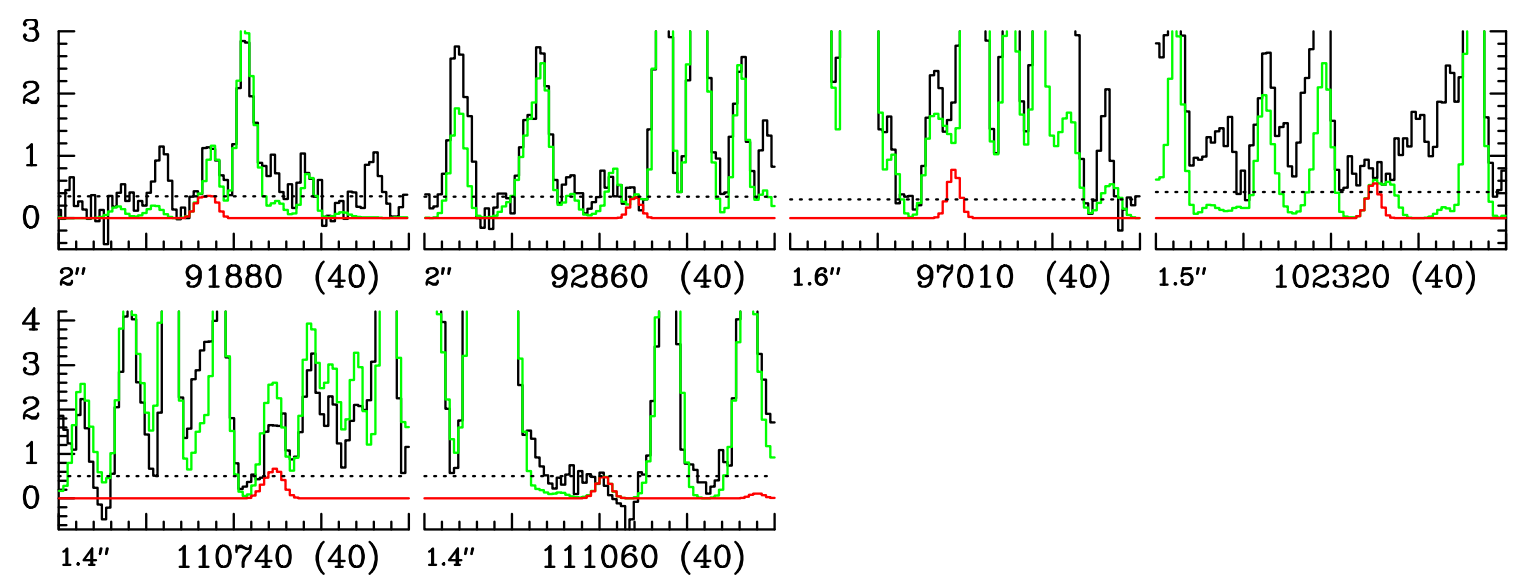

Fig. A.19. Same as Fig. A. 1 for $\mathrm{CH}_{3} \mathrm{CONH}_{2}, \Delta v_{\mathrm{t}} \neq 0$.
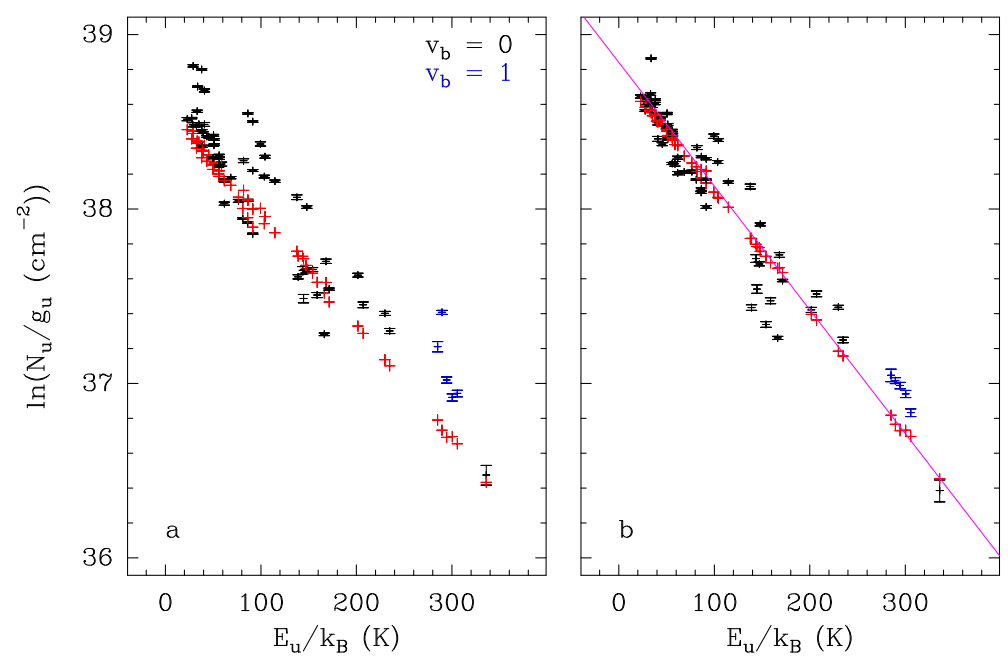

Fig. A.20. Same as Fig. 4 for $\mathrm{CH}_{3} \mathrm{NCO}$.
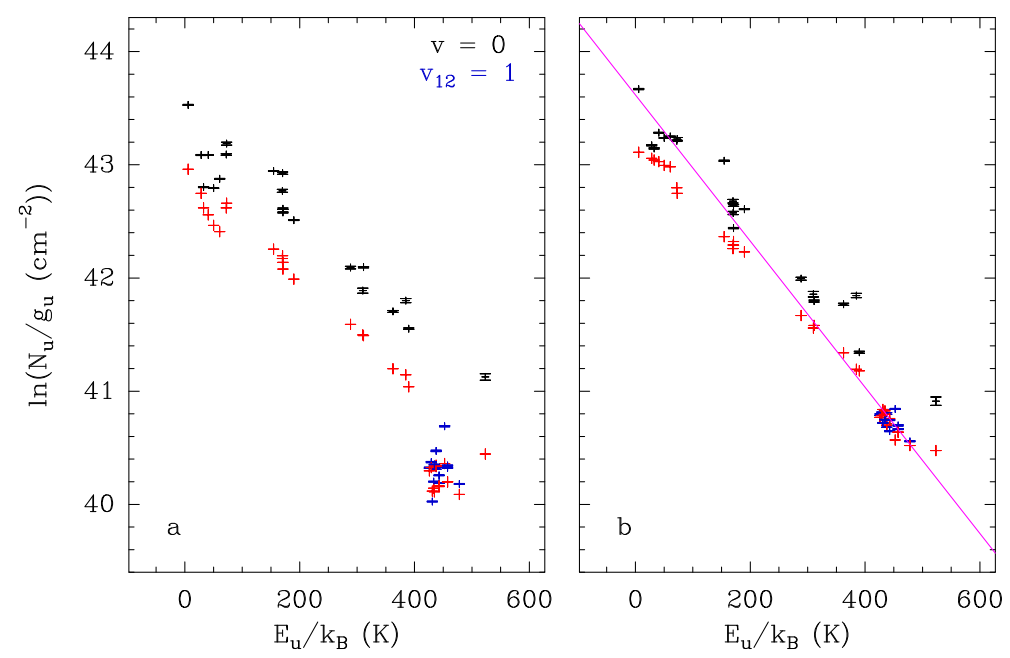

Fig. A.21. Same as Fig. 4 for $\mathrm{NH}_{2} \mathrm{CHO}$. 
A. Belloche et al.: Rotational spectroscopy, tentative interstellar detection, and chemical modeling of N-methylformamide
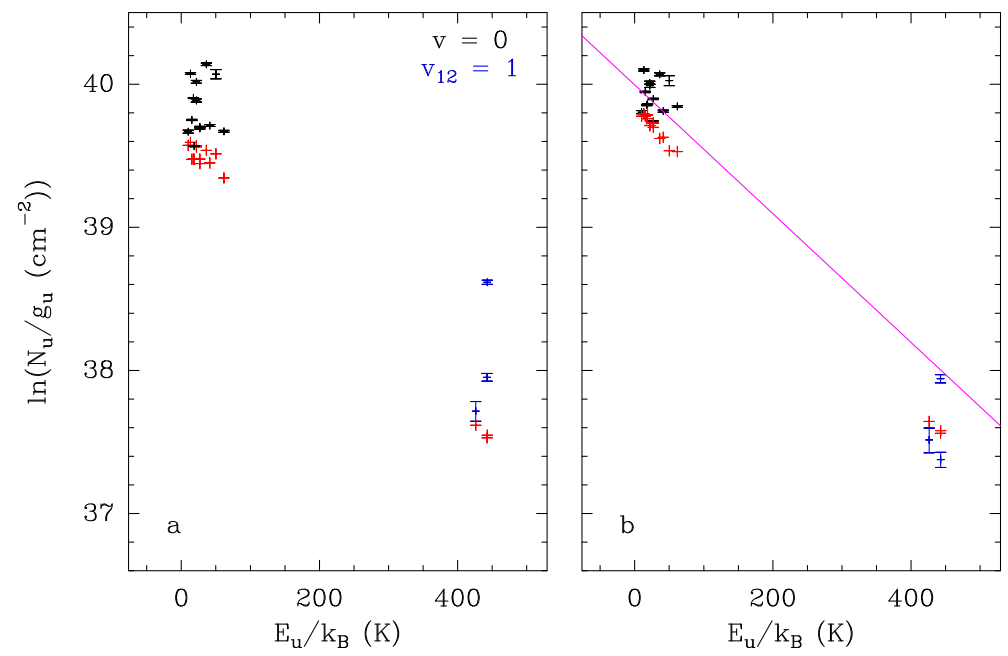

Fig. A.22. Same as Fig. 4 for $\mathrm{NH}_{2}{ }^{13} \mathrm{CHO}$.
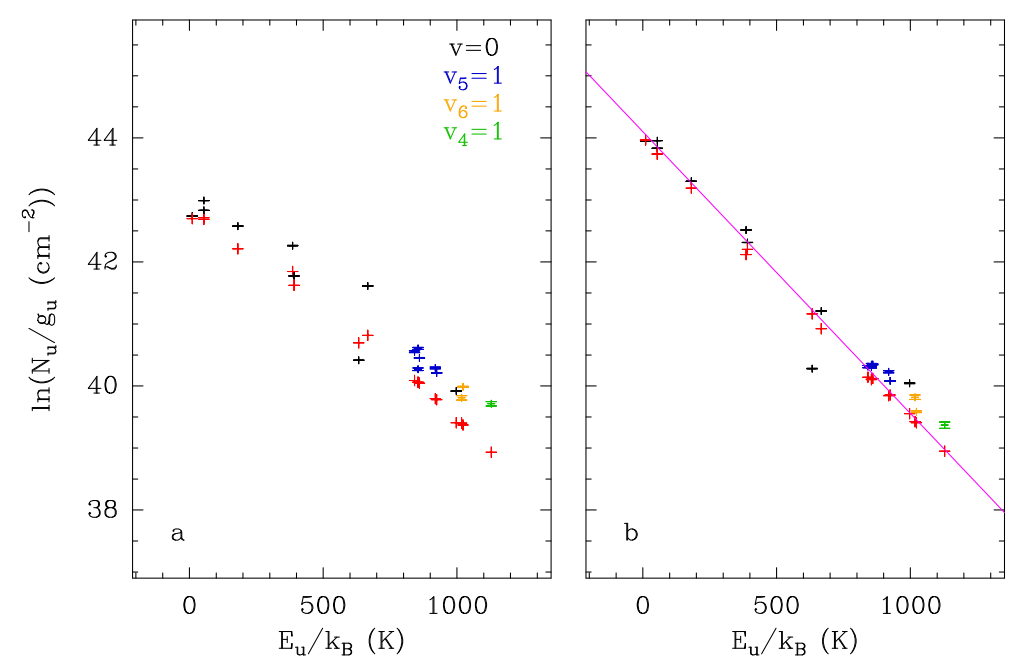

Fig. A.23. Same as Fig. 4 for HNCO.
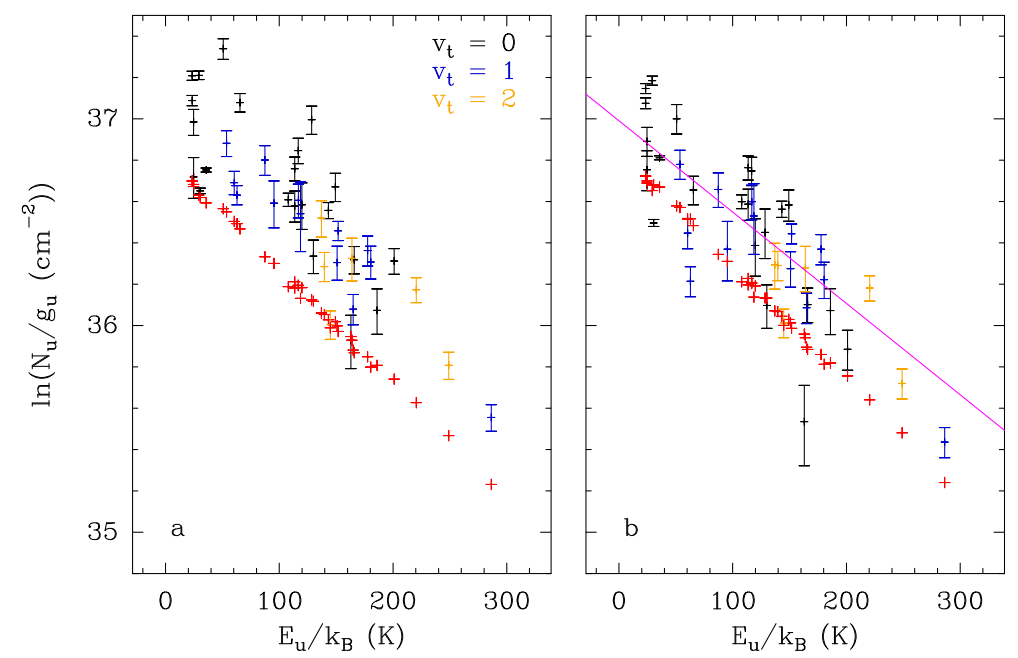

Fig. A.24. Same as Fig. 4 for $\mathrm{CH}_{3} \mathrm{CONH}_{2}$. 


\section{Appendix B: Complementary tables}

Table B.1 provides the direct comparison between low-order spectral parameters determined in this study and the corresponding parameters from Kawashima et al. (2010).

Tables B.2-B.4 list the transitions that are used to build the population diagram of $\mathrm{CH}_{3} \mathrm{NHCHO}$ (Fig. 4). The transitions that we consider as clearly detected individually, that is, that are above the $3 \sigma$ level and do not suffer too much from contamination by other species, are marked with a star. Because there are only five such transitions, the assignment of the corresponding five detected lines to $\mathrm{CH}_{3} \mathrm{NHCHO}$ is only tentative (see Sect. 4.2).

Table B.1. Comparison of the main spectroscopic parameters with previous results.

\begin{tabular}{ccc}
\hline \hline Parameter & This study & Kawashima et al. (2010) \\
\hline$V_{3}\left(\mathrm{~cm}^{-1}\right)$ & $51.7199088(90)$ & $53.91824(11)$ \\
$I_{\alpha}\left(\mathrm{u}^{2}\right)$ & $3.1904202^{a}$ & 3.1542735 \\
$\lambda_{a}$ & 0.91962767 & 0.90814582 \\
$\rho_{a}$ & 0.0744683 & 0.0768384 \\
$A(\mathrm{MHz})$ & $19246.36(12)$ & 19455.9905 \\
$B(\mathrm{MHz})$ & $6376.416(72)$ & 6297.7305 \\
$C(\mathrm{MHz})$ & $4933.8717(42)$ & $4905.1546^{b}$ \\
$\chi_{a a}(\mathrm{MHz})$ & $2.1013(27)$ & $2.115(22)$ \\
$\chi_{b b}-\chi_{c c}(\mathrm{MHz})$ & $5.9666(61)$ & $5.952(28)$ \\
$\chi_{a b}(\mathrm{MHz})$ & $0.2630(23)$ & $0.265(17)$ \\
\hline
\end{tabular}

Notes. ${ }^{(a)}$ Recalculated from $\rho$ value. ${ }^{(b)}$ Average of $A$ - and $E$-state values from Table 2 of Kawashima et al. (2010). 
A. Belloche et al.: Rotational spectroscopy, tentative interstellar detection, and chemical modeling of N-methylformamide

Table B.2. Selection of lines of $\mathrm{CH}_{3} \mathrm{NHCHO} v_{\mathrm{t}}=0$ covered by the EMoCA survey of Sgr B2(N2).

\begin{tabular}{|c|c|c|c|c|c|c|c|c|c|c|c|c|}
\hline Transition $^{a}$ & $\begin{array}{l}\text { Frequency } \\
(\mathrm{MHz})\end{array}$ & $\begin{array}{l}\text { Unc. }^{b} \\
(\mathrm{kHz})\end{array}$ & $\begin{array}{c}E_{\text {up }}^{c} \\
(\mathrm{~K})\end{array}$ & $g_{\text {up }}{ }^{d}$ & $\begin{array}{c}A_{\mathrm{ul}}^{e} \\
\left(10^{-5} \mathrm{~s}^{-1}\right)\end{array}$ & $\begin{array}{c}\sigma^{f} \\
(\mathrm{mK})\end{array}$ & $\tau_{\text {peak }}{ }^{g}$ & \multicolumn{2}{|c|}{ Frequency range $^{h}$} & $\begin{array}{c}I_{\mathrm{obs}^{i}} \\
\left(\mathrm{~K} \mathrm{~km} \mathrm{~s}^{-1}\right)\end{array}$ & $\begin{array}{l}I_{\bmod ^{j}} \\
(\mathrm{~K} \mathrm{~km}\end{array}$ & $\begin{array}{l}I_{\text {all }}^{k} \\
\left.\mathrm{~s}^{-1}\right)\end{array}$ \\
\hline $8_{2,6}-7_{2,5}$ & 91888.760 & 1 & 27 & 17 & 3.1 & 117 & 0.024 & 91887.2 & 91890.1 & $6.2(5)^{\star}$ & 3.6 & 4.0 \\
\hline $9_{0,9}-8_{0,8}$ & 93406.380 & 1 & 23 & 19 & 3.6 & 104 & 0.032 & 93404.9 & 93407.8 & $7.7(4)^{\star}$ & 4.5 & 4.8 \\
\hline $9_{2,8}-8_{2,7}$ & 99695.472 & 1 & 27 & 19 & 4.2 & 162 & 0.033 & 99693.6 & 99697.0 & $10.5(6)^{\star}$ & 7.3 & 7.5 \\
\hline $9_{18}-8_{17}$ & 101309.201 & 1 & 30 & 19 & 4.7 & 133 & 0.034 & 101307.6 & 101310.6 & $11.1(5)$ & 7.5 & 7.7 \\
\hline $10_{6,4}-9_{6,3}$ & 111215.743 & 1 & 59 & 21 & 4.1 & 166 & 0.024 & 111213.9 & 111217.3 & $7.9(6)^{\star}$ & 5.8 & 6.2 \\
\hline $11_{0,11}-1_{0,1}$ & 112875.483 & 1 & 37 & 23 & 6.6 & 242 & 0.044 & 112873.3 & 112877.3 & $22.6(9)$ & 11.7 & 12.8 \\
\hline
\end{tabular}

Notes. ${ }^{(a)}$ Quantum numbers of the upper and lower levels. ${ }^{(b)}$ Frequency uncertainty. ${ }^{(c)}$ Upper level energy. ${ }^{(d)}$ Upper level degeneracy. ${ }^{(e)}$ Einstein coefficient for spontaneous emission. ${ }^{(f)}$ Measured rms noise level. ${ }^{(g)}$ Peak opacity of the synthetic line. ${ }^{(h)}$ Frequency range over which the emission was integrated. ${ }^{(i)}$ Integrated intensity of the observed spectrum in brightness temperature scale. The statistical standard deviation is given in parentheses in unit of the last digit. Values marked with a star indicate the lines that suffer little contamination and are thus unambiguously detected. ${ }^{(j)}$ Integrated intensity of the synthetic spectrum of $\mathrm{CH}_{3} \mathrm{NHCHO} v_{\mathrm{t}}=0 .{ }^{(k)}$ Integrated intensity of the model that contains the contribution of all identified molecules, including $\mathrm{CH}_{3} \mathrm{NHCHO} v_{\mathrm{t}}=0$.

Table B.3. Selection of lines of $\mathrm{CH}_{3} \mathrm{NHCHO} v_{\mathrm{t}}=1$ covered by the EMoCA survey of Sgr B2(N2).

\begin{tabular}{|c|c|c|c|c|c|c|c|c|c|c|c|c|}
\hline Transition $^{a}$ & $\begin{array}{l}\text { Frequency } \\
(\mathrm{MHz})\end{array}$ & $\begin{array}{l}\text { Unc. }^{b} \\
(\mathrm{kHz})\end{array}$ & $\begin{array}{l}E_{\mathrm{up}}^{c} \\
(\mathrm{~K})\end{array}$ & $g_{\text {up }}{ }^{d}$ & $\begin{array}{c}A_{\mathrm{ul}}^{e} \\
\left(10^{-5} \mathrm{~s}^{-1}\right)\end{array}$ & $\begin{array}{l}\sigma^{f} \\
(\mathrm{mK})\end{array}$ & $\tau_{\text {peak }}{ }^{g}$ & \multicolumn{2}{|c|}{ Frequency range ${ }^{h}$} & $\begin{array}{c}I_{\mathrm{obs}}{ }^{i} \\
\left(\mathrm{~K} \mathrm{~km} \mathrm{~s}^{-1}\right)\end{array}$ & $\begin{array}{l}I_{\text {mod }}^{j} \\
(\mathrm{~K} \mathrm{~km}\end{array}$ & $\begin{array}{c}I_{\text {all }}^{k} \\
\left.\mathrm{~s}^{-1}\right)\end{array}$ \\
\hline $9_{1,9}-8_{1,8}$ & 91245.678 & 1 & 71 & 19 & 3.2 & 149 & 0.023 & 91244.4 & 91247.3 & $7.6(6)$ & 4.6 & 5.1 \\
\hline $10_{5,6}-9_{5,5}$ & 99534.218 & 1 & 114 & 21 & 3.9 & 162 & 0.021 & 99532.4 & 99535.3 & $7.9(6)$ & 4.6 & 6.8 \\
\hline $11_{1,11}-1_{0,11}$ & 108750.182 & 1 & 106 & 23 & 7.2 & 115 & 0.036 & 108748.1 & 108751.5 & $15.6(4)$ & 9.2 & 11.9 \\
\hline $11_{1,11}-1_{0,11}$ & 111776.580 & 1 & 81 & 23 & 5.9 & 166 & 0.033 & 111774.6 & 111778.0 & $14.5(6)$ & 8.0 & 9.2 \\
\hline $10_{3,8}-9_{3,7}$ & 113609.358 & 1 & 86 & 21 & 5.3 & 242 & 0.025 & 113607.5 & 113610.9 & $8.7(8)^{\star}$ & 6.4 & 7.1 \\
\hline
\end{tabular}

Notes. Same as Table B.2 but for $\mathrm{CH}_{3} \mathrm{NHCHO} v_{\mathrm{t}}=1$.

Table B.4. Selection of lines of $\mathrm{CH}_{3} \mathrm{NHCHO} v_{\mathrm{t}}=2$ covered by the EMoCA survey of Sgr B2(N2).

\begin{tabular}{|c|c|c|c|c|c|c|c|c|c|c|}
\hline Transition $^{a}$ & $\begin{array}{c}\text { Frequency } \\
(\mathrm{MHz})\end{array}$ & $\begin{array}{l}\text { Unc. }^{b} \\
(\mathrm{kHz})\end{array}$ & $\begin{array}{c}E_{\text {up }}{ }^{c} \\
(\mathrm{~K})\end{array}$ & $g_{\text {up }}{ }^{d}$ & $\begin{array}{c}A_{\mathrm{ul}}^{e} \\
\left(10^{-5} \mathrm{~s}^{-1}\right)\end{array}$ & $\begin{array}{c}\sigma^{f} \\
(\mathrm{mK})\end{array}$ & $\tau_{\text {peak }^{g}}^{g}$ & $\begin{array}{l}\text { Frequency range } \\
(\mathrm{MHz}) \quad(\mathrm{MHz})\end{array}$ & $\begin{array}{c}I_{\mathrm{obs}}{ }^{i} \\
\left(\mathrm{~K} \mathrm{~km} \mathrm{~s}^{-1}\right)\end{array}$ & $\begin{array}{c}I_{\bmod ^{j}} I_{\text {all }}^{k} \\
\left(\mathrm{~K} \mathrm{~km} \mathrm{~s}^{-1}\right)\end{array}$ \\
\hline $10_{0,10}-9_{0,9}$ & 85854.246 & 3 & 147 & 21 & 4.0 & 158 & 0.023 & $85853.0 \quad 85855.4$ & $6.9(6)$ & $\begin{array}{ll}4.1 & 4.7 \\
\end{array}$ \\
\hline
\end{tabular}

Notes. Same as Table B.2 but for $\mathrm{CH}_{3} \mathrm{NHCHO} v_{\mathrm{t}}=2$. 